\title{
Effects of imaging modalities, brain atlases and feature selection on prediction of Alzheimer's disease.
}

\section{$\operatorname{AUTHOR}(\mathrm{S})$ :}

Ota, Kenichi; Oishi, Naoya; Ito, Kengo; Fukuyama, Hidenao

\section{CITATION:}

Ota, Kenichi ... [et al]. Effects of imaging modalities, brain atlases and feature selection on prediction of Alzheimer's disease.. Journal of neuroscience methods 2015, 256: 168-183

\section{ISSUE DATE:}

2015-12-30

URL:

http://hdl.handle.net/2433/202100

\section{RIGHT:}

(c) 2015. This manuscript version is made available under the CC-BY-NC-ND 4.0 license

http://creativecommons.org/licenses/by-nc-nd/4.0/; The full-text file will be made open to the public on 28 February 2017 in accordance with publisher's 'Terms and Conditions for Self-Archiving'., この論文は出版社版でありません。引 用の際には出版社版をご確認ご利用ください。; This is not the published version. Please cite only the published version. 


\section{Effects of imaging modalities, brain atlases and feature selection on prediction of}

\section{Alzheimer's disease}

Kenichi Ota ${ }^{\text {a, }}$, Naoya Oishi ${ }^{\text {a, }{ }^{*}, \text { Kengo Ito }^{\text {d }} \text {, Hidenao Fukuyama }}{ }^{\text {a, }}$, the SEAD-J Study

Group $^{1}$, for the Alzheimer's Disease Neuroimaging Initiative ${ }^{2}$

${ }^{a}$ Human Brain Research Center, Kyoto University Graduate School of Medicine, 54 Shogoin Kawahara-cho, Sakyo-ku, Kyoto 606-8507, Japan

${ }^{\mathrm{b}}$ Center for the Promotion of Interdisciplinary Education and Research, Kyoto University, 54 Shogoin Kawahara-cho, Sakyo-ku, Kyoto 606-8507, Japan

${ }^{c}$ Department of Psychiatry, Kyoto University Graduate School of Medicine, 54 Shogoin Kawahara-cho, Sakyo-ku, Kyoto 606-8507, Japan

${ }^{\mathrm{d}}$ Department of Clinical and Experimental Neuroimaging, National Center for Geriatrics and Gerontology, 7-430 Morioka-cho, Obu-shi, Aichi 474-8511, Japan

${ }^{1}$ Data used in the preparation of this article were obtained from the Research group of the Studies on Diagnosis of Early Alzheimer's Disease-Japan (SEAD-J), which comprised investigators from nine different facilities. As such, the investigators within the SEAD-J study group contributed to the design and implementation of SEAD-J and/or provided data but did not participate in the analysis or writing of this report.

${ }^{2}$ Data used in preparation of this article were obtained from the Alzheimer's Disease Neuroimaging Initiative (ADNI) database (adni.loni.usc.edu). As such, the investigators within the ADNI contributed to the design and implementation of ADNI and/or provided data but did not participate in analysis or writing of this report. A complete listing of ADNI 
investigators can be found at: http://adni.loni.usc.edu/wpcontent/uploads/how_to_apply/ADNI_Acknowledgement_List.pdf.

* Corresponding author at: Human Brain Research Center, Kyoto University Graduate School of Medicine, 54 Shogoin-Kawahara-cho, Sakyo-ku, Kyoto 606-8507, Japan. E-mail address: noishi@kuhp.kyoto-u.ac.jp (N. Oishi).

TEL: $+81-75-751-3695$.

FAX: $+81-75-751-3202$.

Abbreviations: AAL, Automated Anatomical Labeling; A $\beta$, amyloid- $\beta$; AD, Alzheimer's disease; ADAS-J cog, Alzheimer's Disease Assessment Scale-Cognitive Subscale, Japanese version; ADNI, Alzheimer's Disease Neuroimaging Initiative; aMCI, amnestic mild cognitive impairment; ANCOVA, analysis of covariance; ANOVA, analysis of variance; AUC, area under the curve; CMRglc, cerebral metabolic rate for glucose; FDG-PET, 18Ffluorodeoxyglucose positron emission tomography; GDS, Geriatric Depression Scale; GM, gray matter; LOOCV, leave-one-out cross-validation; LPBA40, LONI Probabilistic Brain Atlas; MCI, mild cognitive impairment; MCI-C, MCI converter; MCI-NC, MCI nonconverter; MMSE, Mini-Mental State Examination; MR, magnetic resonance; MRI, magnetic resonance imaging; MSVM-RFE, multiple support vector machine recursive feature elimination; NFT, neurofibrillary tangle; PVE, partial volume effects; RFE, recursive feature elimination, ROC, receiver operating characteristic; ROI, region of interest; SEAD-J, Studies on Diagnosis of Early Alzheimer's Disease-Japan; SVM, support vector machine; VBM, voxel-based morphometry; WMH, white matter hyperintensity; WMS-R-LM, Wechsler Memory Scale-Revised Logical memory test. 


\section{Abstract \\ -Background}

The choice of biomarkers for early detection of Alzheimer's disease (AD) is important for improving the accuracy of imaging-based prediction of conversion from mild cognitive impairment (MCI) to AD. The primary goal of this study was to assess the effects of imaging modalities and brain atlases on prediction. We also investigated the influence of support vector machine recursive feature elimination (SVM-RFE) on predictive performance. -Methods

Eighty individuals with amnestic MCI [40 developed AD within 3 years] underwent structural magnetic resonance imaging (MRI) and ${ }^{18} \mathrm{~F}$-fluorodeoxyglucose positron emission tomography (FDG-PET) scans at baseline. Using Automated Anatomical Labeling (AAL) and LONI Probabilistic Brain Atlas (LPBA40), we extracted features representing gray matter density and relative cerebral metabolic rate for glucose in each region of interest from the baseline MRI and FDG-PET data, respectively. We used linear SVM ensemble with bagging and computed the area under the receiver operating characteristic curve (AUC) as a measure of classification performance. We performed multiple SVM-RFE to compute feature ranking. We performed analysis of variance on the mean AUCs for eight feature sets.

\section{-Results}

The interactions between atlas and modality choices were significant. The main effect of SVM-RFE was significant, but the interactions with the other factors were not significant.

\section{-Comparison with Existing Method}

Multimodal features were found to be better than unimodal features to predict AD. FDG-PET was found to be better than MRI.

-Conclusions 
Imaging modalities and brain atlases interact with each other and affect prediction. SVMRFE can improve the predictive accuracy when using atlas-based features.

Keywords: Alzheimer's disease; mild cognitive impairment; ${ }^{18} \mathrm{~F}$-fluorodeoxyglucose positron emission tomography; magnetic resonance imaging; support vector machine; feature selection.

\section{Introduction}

Alzheimer's disease (AD), which is the main cause of dementia, is a slowly progressive neurodegenerative disorder that leads to declines in memory and other cognitive abilities (Alzheimer's Association, 2013). The revised diagnostic criteria and guidelines for AD (Jack et al., 2011) proposed three stages of AD, including preclinical AD (Sperling et al., 2011), mild cognitive dementia (MCI) due to AD (Albert et al., 2011), and dementia due to AD (McKhann et al., 2011). MCI is a heterogeneous clinical entity that pertains to characteristics between those associated with normal aging and $\mathrm{AD}$, and some individuals with $\mathrm{MCI}$ develop AD later (Petersen et al., 1999, 2001).

Among the neuropathological hallmarks of AD, neurofibrillary tangles (NFTs), and senile plaques are considered essential for neuropathological diagnosis of $\mathrm{AD}$ (Hyman et al., 2012). NFTs are, at least initially, intraneuronal fibrils primarily composed of hyperphosphorylated tau protein, whereas senile plaques are extracellular deposits of amyloid- $\beta(\mathrm{A} \beta)$ peptides. Progression of these AD neuropathological changes probably begins decades before the onset of cognitive decline (Mufson et al., 2012). Early detection of AD is, therefore, important as a basis for early intervention with disease-modifying drugs (Giacobini and Gold, 2013). 
Three imaging biomarkers, as biomarkers to identify brain changes that precede the earliest symptoms, are included in the research criteria for diagnosis of MCI due to AD (Albert et al., 2011). Positron emission tomography (PET) amyloid imaging can measure and visualize A $\beta$ deposition. Hippocampal volume or medial temporal atrophy on magnetic resonance imaging (MRI) and brain glucose hypometabolism on ${ }^{18} \mathrm{~F}$-fluorodeoxyglucose (FDG)-PET imaging are measures reflecting neuronal injury, namely, general damage to neurons and synapses (Jack and Holtzman, 2013). Besides structural MRI and FDG-PET, imaging techniques reflecting neuronal injury include single photon emission tomography (SPECT) perfusion imaging (Ito et al., 2013), diffusion tensor imaging (DTI) (Oishi et al., 2011), functional MRI (fMRI) (Li et al., 2015), and MRI perfusion (Chao et al., 2010). Among these, available data for MRI-related biomarkers except structural MRI are limited and less validated. These neuronal injury markers on MRI or FDG-PET are considered to be less direct or nonspecific evidence of $\mathrm{AD}$, not direct evidence of the presence of $\mathrm{A} \beta$ or tau (Albert et al., 2011).

However, these markers are considered to be associated with synaptic loss, which is one of the major neuropathological findings in the brains of individuals with early AD (Scheff et al., 2006). Synaptic loss and neuronal loss are the major pathological substrates of cortical atrophy (Serrano-Pozo et al., 2011) and correlates with cognitive decline(Terry et al., 1991). Longitudinal progression of cognitive decline correlates brain glucose metabolic changes (Shokouhi et al., 2013). Synaptic loss occurs in the limbic regions and the neocortex in individuals with amnestic MCI (aMCI) (Scheff and D. A. Price, 2006). Different from the distribution of $A \beta$ deposition, temporospatial accumulation of NFTs originates in the entorhinal cortex constituting the anterior portion of the parahippocampal gyrus and extends through the limbic regions to the neocortex (Braak and Braak, 1991). 
Structural MRI and FDG-PET are topographical biomarkers that can help to characterize clinical subtypes with distinct regional patterns of cortical hypometabolism in FDG-PET and of cortical atrophy on structural MRI. FDG-PET has good sensitivity in detection of early brain dysfunction in AD among topographical markers (Dubois et al., 2014). In addition, structural MRI and FDG-PET are less invasive than CSF biomarkers and less expensive than Amyloid PET imaging. On the basis of these foundations, MRI and FDG-PET, particularly their multimodal combination (Price, 2012), can provide valuable biomarkers for early detection of AD. FDG-PET abnormalities are known to precede any cognitive symptoms in individuals who later develop AD (Jack et al., 2010). However, the relative diagnostic abilities of MRI and FDG-PET and of combinations of these different modalities for early disease detection remain controversial (Karow et al., 2010; Mosconi et al., 2006). To achieve scientific evidence of the diagnostic utility of FDG-PET and of MRI in early diagnosis of AD, the Studies on Diagnosis of Early Alzheimer's Disease_-Japan (SEAD-J) (Kawashima et al., 2012) was launched in 2005 along with other multicenter clinical trials.

Predictive models based on machine learning algorithms have been widely used for MCI classification (Cuingnet et al., 2011; Young et al., 2013; Zhang et al., 2011). The choice of distinguishing features has an important role in pattern classification (Duda et al., 2001). Atlas-based parcellation using a predefined anatomical brain atlas is a simple feature extraction method with good interpretability and general versatility (Cuingnet et al., 2011; Zhang et al., 2011). Because of the brain atlas concordance problem (Bohland et al., 2009), the use of different brain atlases for parcellation provides different features and can affect the ability to predict conversion from MCI to AD. We have recently reported the importance of the choice of brain atlases for feature extraction in the prediction of conversion by using 
atlas-based MR biomarkers (Ota et al., 2014). Differences in imaging modalities can also affect predictive performance. However, the effects of imaging modalities and brain atlases for feature extraction on $\mathrm{AD}$ prediction have not been well documented.

In addition to feature extraction, feature selection is also important in view of dimension reduction for improving generalization ability and identifying distinguishing features. The effects of feature selection on $\mathrm{AD}$ predictive performance remain controversial (Chu et al., 2012; Cuingnet et al., 2011; Kerr et al., 2014). Our previous results (Ota et al., 2014) for MRbased features suggest that support vector machine (SVM)-based recursive feature elimination (RFE) can be important in AD prediction. However, the effects of the use of FDG-PET features or multimodal combination of MRI and FDG-PET features on feature selection have not been clarified.

The primary goal of this study was to assess the effects of imaging modalities and of brain atlases and their interactions on $\mathrm{AD}$ prediction. We performed atlas-based feature extraction from MRI and FDG-PET data by using different brain atlases. Using these unimodal or multimodal imaging feature sets, we performed SVM-based classification of MCI and added SVM-RFE feature selection to also evaluate the influence of feature selection on the classification performance.

\section{Materials and methods}

\section{Participants}


We identified 80 individuals with aMCI from a total of 114 participants in the SEAD-J (Ito et al., 2015). Diagnosis of MCI was based on an interview with neurologists as described previously (Ota et al., 2014). "Conversion" was defined as a change in diagnosis from aMCI to $\mathrm{AD}$ during a 3-year follow-up period, and 40 participants $(50 \%)$ converted to $\mathrm{AD}$ within 3 years. We excluded 34 participants from the analysis for the following reasons: two participants did not undergo baseline three-dimensional T1-weighted MRI scans, three participants converted to non-AD dementia (vascular dementia, dementia with Lewy bodies, and frontotemporal dementia), 23 participants withdrew from the study within 3 years, and six participants were excluded because of the lack of whole-brain coverage in their baseline T1-weighted MRI scans. Table 1 shows more details on the participants' characteristics at baseline. The converter group (MCI-C) and nonconverter group (MCI-NC) significantly differed in baseline neuropsychological scores, including the Mini-Mental State Examination (Folstein et al., 1975) $(p<0.0009, \mathrm{r}=0.36)$, Alzheimer's Disease Assessment ScaleCognitive Subscale, Japanese version (Homma et al., 1992) $(p=0.025, \mathrm{r}=0.25)$, the Wechsler Memory Scale-Revised Logical memory test (WMS-R-LM) (Sullivan, 1996) (immediate recall: $p<10^{-4}, \mathrm{r}=0.42$; delayed recall: $p<10^{-5}, \mathrm{r}=0.48$ ), and Geriatric Depression Scale (Nyunt et al., 2009; Yesavage et al., 1982) $(p=0.006, \mathrm{r}=0.30)$. No significant differences were observed in age $(p=0.55, \mathrm{r}=0.068)$, gender $\left(\chi^{2}\right.$ test, $p=0.82, \mathrm{w}$ $=0.025)$, and education $(p=0.46, \mathrm{r}=0.084)$. Student's $t$-test was used to compare baseline differences between the two groups unless otherwise stated. We computed $r$ for the t-test and w for the $\chi^{2}$ test as measures of effect size (Cohen, 1992).

Magnetic resonance image acquisition and preprocessing 
Three-dimensional structural MRI scans at the baseline were collected by using T1-weighted gradient echo sequences on a variety of 1.5-T MRI scanners at eight sites and a 3.0-T MRI scanner at one site. Of 80 participants, 77 (96\%) participants were scanned on the 1.5-T scanners and $3(4 \%)$ on the 3-T scanner. Details about MRI acquisition parameters are provided in Supplementary Table 2. We performed the following voxel-based morphometry (VBM) preprocessing procedures using the SPM8 software package (http://www.fil.ion.ucl.ac.uk/spm/software/spm8) and VBM8 Toolbox (Kurth et al., 2010) (http://dbm.neuro.uni-jena.de/vbm) in MATLAB 7.12. We performed VBM procedures including segmentation, spatial normalization, and smoothing as described previously (Ota et al., 2014).

Fluorodeoxyglucose positron emission tomography image acquisition and preprocessing

FDG-PET scans were collected in a resting state in a dark room 40-60 min after venous injection of ${ }^{18}$ F-FDG (Kawashima et al., 2012). The whole brain was used as the reference region for intensity normalization to compare the effects of two different brain atlases on the ability of prediction because of its higher signal-to-noise ratio compared with intensity normalization to the cerebellum (Dukart et al., 2010). The average whole brain uptake of the converters was $10143 \pm 10289$, that of nonconverters, $8451 \pm 10912,(t$-test, $p=0.48$, Cohen's $d=0.11$ ). The images were normalized by using an in-house FDG-PET template created from FDG-PET and MRI brain scans of 23 normal elderly individuals. Partial volume effects (PVE) were not corrected according to a previous finding that PVE correction did not affect the detection of hippocampal hypometabolism in aMCI when using the global mean for scaling (Mevel et al., 2007). The resulting images were smoothed by using an 8-mm isotropic 
Gaussian filter to blur individual variations in gyral anatomy and to increase the signal-tonoise ratio (Mevel et al., 2007).

\section{Voxel-based comparison}

To determine the differences in MRI and FDG-PET data between converters and nonconverters, we performed voxel-based comparison of gray matter (GM) maps and of FDG-PET images between converters and nonconverters by using the SPM8 software package. We used analysis of covariance (ANCOVA) models with age, gender, and scan sites as covariates according to a previous report (Ota et al., 2014). The thresholds for the statistical parametric maps were set to $p=0.001$, uncorrected for multiple comparisons at a voxel level, and $p=0.05$, family-wise error corrected at a cluster level. Clusters were visualized by using the BrainNet Viewer 1.43 (http://www.nitrc.org/projects/bnv/) (Xia et al., 2013).

\section{Feature extraction}

To assess the effects of brain atlases for template-based region-of-interest (ROI) analysis on the performance of prediction, we compared two brain atlases for simplicity: the Automated Anatomical Labeling (AAL) atlas (Tzourio-Mazoyer et al., 2002) and the LONI Probabilistic Brain Atlas (LPBA40) (Shattuck et al., 2008) (Fig. 1). The AAL atlas has 116 ROIs, including 90 cerebral regions and 26 cerebellar regions, whereas the LPBA40 has 56 ROIs, including 54 cerebral regions, the brainstem, and the cerebellum. Using these two brain atlases, we extracted feature vectors from the preprocessed baseline MRI, which was modulated with the Jacobian, and from the FDG-PET data, as described previously (Ota et al., 
2014) Accordingly, we obtained eight different feature sets, including two MR-based features (MRI-AAL and MRI-LPBA40) representing GM density in each ROI, two PET-based features (PET-AAL and PET-LPBA40) representing relative cerebral metabolic rate for glucose in each ROI, and four multimodal features (MRI-AAL + PET-AAL, MRI-AAL + PET-LPBA40, MRI-LPBA40 + PET-AAL, and MRI-LPBA40 + PET-LPBA40).

\section{Classification}

Fig. 1 is a schematic overview of our classification pipeline. SVMs (Vapnik, 1998) are one of the most popular supervised learning models and have been applied to the prediction of conversion from MCI to AD (Chu et al., 2012; Ota et al., 2014). To focus on comparison across imaging modalities and brain atlases for feature extraction, we used linear SVMs with the regularization parameter $\mathrm{C}$ of 1 for computational simplicity in the present study as described in a prior study (Zhang et al., 2011).

To enable statistical analysis of the results, we used a bootstrap aggregating (bagging) method (Breiman, 1996) within each round of LOOCV (Dosenbach et al., 2010). In each LOOCV loop, the original data set having 80 participants was divided into a test set of one participant and a training set comprising 79 participants. Then a bootstrap sample of size 72 ( $90 \%$ of the total number of participants) was obtained by bootstrap resampling of the original training set. In general, a smaller bootstrap sample size provides a better prediction performance and a greater variance. A sample size of 72 was determined arbitrarily with the aim of achieving a smaller variance. This procedure was repeated nine times to obtain a bagged ensemble of nine linear SVMs. The number of subsamples was determined empirically in view of a trade-off between computation time and classification performance. 
These multiple linear SVMs were trained on different subsamples of training data to compute the weight vectors and the feature ranking scores, as mentioned below. The final prediction of the bagged ensemble for a given test set was determined by majority voting (Valentini et al., 2004). The final decision value of the bagged ensemble for a test vector was computed by averaging the decision values for the SVMs that voted for the majority class. After each round of LOOCV, we obtained decision values for all participants with regard to a given number of features.

On the basis of these decision values, we generated the receiver operating characteristic (ROC) curve (Metz, 1978) and computed the area under the ROC curve (AUC) as a measure of the performance of a classifier (Fawcett, 2004; Huang and Ling, 2005) by using the pROC package for R (Robin et al., 2011). Other measures, such as accuracy, sensitivity, and specificity with regard to a given number of features, were also computed. We implemented the above classification algorithm by using the e1071 package for R (Dimitriadou et al., 2005) based on the LIBSVM library (Chang and Lin, 2011).

For the eight different feature sets, we repeated the above LOOCV procedure 20 times and computed mean AUC values for AD prediction across $20 \mathrm{LOOCV}$ tests. To compare the ability of prediction across different feature sets, we formed averaged ROC curves with 95\% confidence intervals for each feature set on the basis of the results of 20 LOOCV tests by using Fawcett's vertical averaging algorithm (Fawcett, 2004).

\section{Feature selection}


In our previous study (Ota et al., 2014), we used the SVM-RFE feature selection method (Guyon et al., 2002) to improve the ability of prediction and to identify the regions with high discriminating power. In the present study, we newly applied the multiple SVM-RFE (MSVM-RFE) feature selection method (Duan et al., 2005) to compute the feature ranking score for a given feature from weight vectors of multiple linear SVMs trained on bootstrap subsamples of the original training data.

As described above, we had nine linear SVMs trained on different subsamples of the original training data. Let $w_{j}$ denote the weight vector of the $j$ th linear SVM and $w_{j i}$ be the corresponding weight value associated with the $i$ th feature. The ranking criterion for the $i$ th feature $v_{j i}$ was given as $v_{j i}=\left(w_{j i}\right)^{2}$. As a result of the majority voting, we selected the ranking criteria $v_{k}$ for a feature set $S_{k}$, where $k$ represents the indices of the linear SVMs that voted for the majority class. The feature ranking scores $c_{k}$ for a feature set $S_{k}$ was computed as:

$$
c_{k}=\frac{\overline{v_{k}}}{\sigma_{k}}
$$

where $\overline{v_{k}}$ is the mean of $v_{k}$ and $\sigma_{\mathrm{k}}$ is the standard deviation of $v_{k}$.

On the basis of these feature-ranking scores, the feature with the smallest ranking score was removed, and the feature set was updated at the end of each SVM-RFE loop. After performing $20 \mathrm{LOOCV}$ tests, the final average rank was determined as a mean of 20 average ranks for the respective LOOCV tests. Our implementation of the MSVM-RFE algorithm in $\mathrm{R}$ was adapted from http://www.uccor.edu.ar/paginas/seminarios/Software/SVM_RFE_R_implementation.pdf. Selected regions were visualized by using the BrainNet Viewer Version 1.43 (http://www.nitrc.org/projects/bnv/) (Xia et al., 2013). 


\section{Statistical analysis}

To test the differences between the means of AUC values for eight feature sets, we used $\mathrm{AUC}_{\mathrm{RFE}}$ - and $\mathrm{AUC}_{\mathrm{RFE}}$ for each feature set. $\mathrm{AUC}_{\mathrm{RFE}-}$ for a feature set was an $\mathrm{AUC}$ value that was obtained with the original features (with no feature selection), and $\mathrm{AUC}_{\mathrm{RFE}+}$ was determined as the highest AUC during the SVM-RFE procedure for the feature set. To assess the main effects of different factors (SVM-RFE feature selection, brain atlases for parcellation, and imaging modalities) and interactions among them on the performance of prediction, we initially performed an overall three-way factorial analysis of variance (ANOVA) that included all the factors. The $2 \times 4 \times 3$ ANOVA design included factor "RFE" with levels "RFE-" and "RFE+," factor "atlas" with levels, "MRI-AAL + PET-AAL," “MRI-AAL + PET-LPBA40," "MRI-LPBA40 + PET-AAL," and "MRI-LPBA40 + PETLPBA40," and factor "modality" with levels "MRI," "PET," and "MRI + PET." Then twoway ANOVAs (atlas $\times$ modality) at each level of factor RFE were performed. To examine the effects of atlas and modality in unimodal features, we performed an additional two-way (atlas $\times$ modality) ANOVA at each level of RFE for four unimodal feature sets. Furthermore, to assess the effects of atlas combinations in multimodal feature sets, we performed a twoway (MRI atlas $\times$ PET atlas) ANOVA for four multimodal feature sets. We performed posthoc analysis of interactions by using tests of simple main effects (one-way ANOVA) or Tukey's test, where appropriate. Post-hoc multiple comparisons were performed by using Tukey's test. We performed Type II analysis by using the car package for R (Fox and Weisberg, 2009) to compute sums of squares for ANOVA (Langsrud, 2003). To estimate effect sizes, we computed $\eta^{2}$ as the ratio of sum of squares for an effect to the total sum of squares (Cohen, 1973). Values of $p<0.05$ were considered to indicate statistical significance. The R Statistical Computing Environment, version 3.0.2 (R Development Core Team, 2013) 
was used for classification, feature selection, and all statistical analyses except voxel-based comparisons.

\section{Results}

Voxel-based comparison of gray matter volume and cerebral metabolic rate of glucose

As shown in Table 2, we found one significant cluster of GM reduction in the MCI-C group compared with MCI-NC on MRI and three significant clusters of cerebral glucose hypometabolism on FDG-PET. Fig. 2 shows the locations of the clusters mapped onto the surface of the brain. GM loss in the MCI-C group was found in the left parahippocampal gyrus and the left hippocampus. Brain glucose hypometabolism in the MCI-C group was found mostly in the left temporoparietal association cortex, including the precuneus, inferior temporal gyrus, middle temporal gyrus, and angular gyrus. Reverse contrast (MCI-NC < MCI-C) showed no significant regions on MRI or FDG-PET.

Effects of imaging modality, brain atlas, and support vector machine recursive feature elimination on prediction

Fig. 3 shows the plots of the mean AUC values of different feature sets versus the number of features. The arrows on the top of each plot indicate the number of features that provided the highest $\mathrm{AUC}\left(\mathrm{AUC}_{\mathrm{RFE}+}\right)$ on each curve in the same color. In general, particularly in the multimodal feature sets, the AUC at the right end of each plot (no feature selection) tended to be the highest, whereas the plots of LPBA40-based features, particularly in the unimodal 
feature sets, showed a local maximum where the number of features was $<25$. Tests for the statistical significance of the difference between mean AUCs are described below.

Fig. 4 shows the ROC curves of eight different feature sets for MCI classification. For the MRI-based feature sets, the ROC curve of LPBA40 (shown in red) showed higher sensitivity than that of AAL (shown in green) at almost all specificities. For FDG-PET, in contrast, the ROC curve of AAL (shown in green) demonstrated higher sensitivity than that of LPBA40 (shown in red) at the lower specificity range [false positive rate (FPR) range of 0.25-0.9]. In the multimodal feature sets, MRI-AAL + PET-AAL (shown in green) demonstrated higher sensitivity than any of the other feature sets in the FPR range of values $>0.65$. MRI-LPBA40 + PET-AAL (shown in orange) showed higher sensitivity than did the other combinations, including unimodal features in the FPR range of values $<0.2$.

Table 3 shows the mean AUC and number of features for each feature set for use in our three-way ANOVA. Fig. $5 \mathrm{~A}$ shows a bar plot of the mean $\mathrm{AUC}_{\mathrm{RFE}-}$ and $\mathrm{AUC}_{\mathrm{RFE}}+$ values. $\mathrm{As}$ shown in Table 4, there were significant main effects for three factors: $\operatorname{RFE}[F(1,456)=$ $\left.20.1, p<10^{-5}, \eta^{2}=0.0065\right]$; atlas $\left[F(3,456)=333.0, p<10^{-15}, \eta^{2}=0.32\right]$; and modality $[F$ $\left.(2,456)=466.3, p<10^{-15}, \eta^{2}=0.30\right]$. We found no significant three-way interactions $[F(6$, $\left.456)=1.5, p=0.19, \eta^{2}=0.0028\right]$. The two-way interactions between RFE and atlas $[F(3$, $\left.456)=1.3, p=0.26, \eta^{2}=0.0013\right]$ and between RFE and modality $[F(2,456)=1.1, p=0.33$, $\left.\eta^{2}=0.0007\right]$ were not significant, whereas the two-way interaction between atlas and modality $\left[F(6,456)=111.7, p<10^{-15}, \eta^{2}=0.22\right]$ was significant.

Our post-hoc analysis of the atlas $\times$ modality interaction demonstrated significant simple main effects of atlas at different levels of modality $\left[F(3,456)=227.7, p<10^{-15}, \eta^{2}=0.22\right.$ at 
M1 $(\mathrm{MRI}) ; F(3,456)=137.4, p<10^{-15}, \eta^{2}=0.13$ at M2 $(\mathrm{PET})$; and $F(3,456)=191.2, p<$ $10^{-15}, \eta^{2}=0.19$ at M3 (MRI + PET) ] and significant simple main effects of modality at different levels of atlas $\left[F(2,456)=449.3, p<10^{-15}, \eta^{2}=0.29\right.$ at A1 (MRI-AAL+PET$\mathrm{AAL}) ; F(2,456)=44.9, p<10^{-15}, \eta^{2}=0.029$ at A2 (MRI-AAL+PET-LPBA40); $F(2,456)=$ $100.5, p<10^{-15}, \eta^{2}=0.065$ at A3 (MRI-LPBA40+PET-AAL); and $F(2,456)=206.6, p<$ $10^{-5}, \eta^{2}=0.13$ at A4 (MRI-LPBA40+PET-LPBA40)].

Regarding atlas combinations, post-hoc multiple comparisons for factor atlas revealed significant differences between any of the four atlas combinations $\left(p<10^{-7}\right)$ except between A1 (MRI-AAL + PET-AAL) and A4 (MRI-LPBA40 + PET-LPBA40) $(p=0.40)$. Consequently, marginal means of four groups of atlas combination were ranked in the following order: A3 (MRI-LPBA40 + PET-AAL, 0.724) > A1 (MRI-AAL + PET-AAL, 0.704) and A4 (MRI-LPBA40 + PET-LPBA40, 0.701) > A2 (MRI-AAL + PET-LPBA40, 0.668). Multiple comparisons for atlas at each modality revealed significant differences between any of the different groups $\left(p<10^{-7}\right)$ except between A1 (MRI-AAL + PET-AAL) and A3 (MRI-LPBA40 + PET-AAL) $(p=0.84)$. Accordingly, we observed the following rankings for atlas at each modality: at M1 (MRI), LPBA40 > AAL; at M2 (PET), AAL > LPBA40; and at M3 (MRI + PET), A3 (MRI-LPBA40 + PET-AAL) and A1 (MRI-AAL + PET-AAL) > B4 (MRI-LPBA40 + PET-LPBA40) > A2 (MRI-AAL + PET-LPBA40).

Post-hoc multiple comparisons for modality also demonstrated significant differences between MRI and PET, between MRI and MRI + PET, and between PET and MRI + PET, as shown in Fig. 5B. Accordingly, marginal means of three levels of modality were ranked in the following order: MRI + PET (0.727) > PET (0.688) > MRI (0.684). Multimodal feature sets were superior to unimodal feature sets, and PET-based feature sets were better than MRI- 
based feature sets. Multiple comparisons for modality at each level of factor atlas revealed significant differences between any of the groups $(p<0.001$, except $p=0.02$ for between MRI-AAL and PET-LPBA40 at A2) except between MRI-LPBA40 and PET-AAL at A3 ( $p=$ 0.74). Consequently, the following rankings for modality at each level of atlas were obtained: at A1, M3 (MRI-AAL + PET-AAL) > M2 (PET-AAL) > M1 (MRI-AAL); at A2, M3 (MRIAAL + PET-LPBA40) $>$ M2 (PET-LPBA40) $>$ M1 (MRI-AAL); at A3, M3 (MRI-LPBA40 + PET-AAL) > M2 (PET-AAL) and M1 (MRI-LPBA40); and at A4, M3 (MRI-LPBA40 + PET-LPBA40) > M1 (MRI-LPBA40) > M2 (PET-LPBA40). In summary, multimodal combinations were superior to single unimodal feature sets at all levels of atlas. With regard to unimodal features, PET was better than MRI when using AAL, whereas MRI was better than PET when using LPBA40.

Because the lack of significant three-way $(\mathrm{RFE} \times$ atlas $\times$ modality $)$ interactions indicated that the atlas $\times$ modality interaction was not significantly different depending on the levels of RFE (RFE - and RFE + ), we focused on the atlas $\times$ modality interaction at RFE for simplicity. Our additional two-way (atlas $\times$ modality) ANOVA for comparing the effects of atlas and modality in unimodal feature sets at RFE- demonstrated a significant main effect of modality $\left[F(1,76)=5.2, p=0.02, \eta^{2}=0.008\right]$, whereas we found no significant main effect of atlas as shown in Fig. 5C. In contrast, the interaction between atlas and modality was significant (Fig. 5D). Our post-hoc analysis of the atlas $\times$ modality interaction revealed the significant simple main effects of modality at AAL and at LPBA40, and of atlas at MRI and at PET.

Another two-way (MRI atlas $\times$ PET atlas) ANOVA for comparing the effects of atlases in multimodal feature sets at RFE- showed significant main effects of MRI atlas $[F(1,76)=$ 191.6, $\left.p<10^{-15}, \eta^{2}=0.17\right]$ and PET atlas $\left[F(1,76)=716.0, p<10^{-15}, \eta^{2}=0.62\right]$, and as 
shown in Fig. 5E, we also found significant interactions between these factors. Our post-hoc analysis of the interaction demonstrated significant simple main effects of MRI atlas at LPBA40 for PET, of PET atlas at AAL for MRI, and of PET atlas at LPBA40 for MRI. We found no significant simple main effect of MRI atlas at AAL for PET.

According to our analysis, multimodal features generally yielded better AUC values than did unimodal features. Comparisons of mean $\mathrm{AUC}_{\mathrm{RFE}-\mathrm{values}}$ for multimodal feature sets indicated the following ranking: MRI-LPBA40 + PET-AAL (0.748) and MRI-AAL + PETAAL $(0.747)>$ MRI-LPBA40 + PET-LPBA40 (0.725) > MRI-AAL + PET-LPBA40 (0.680) (Tukey’s test, $p<10^{-7}$ except for $p=0.997$ for a pair between MRI-LPBA40 + PET-AAL and MRI-AAL + PET-AAL). Regarding MRI-based unimodal features, MRI-LPBA40 (0.705) was significantly better than MRI-AAL $(0.655)\left(p<10^{-7}\right)$. In contrast, PET-AAL (0.709) was significantly superior to PET-LPBA40 (0.661) $\left(p<10^{-7}\right)$. Comparisons between the unimodal data of MRI and FDG-PET revealed no significant differences between MRIAAL and PET-LPBA40 $(p=0.32)$ or between MRI-LPBA40 and PET-AAL $(p=0.91)$. No significant differences were observed between any of the other pairs $\left(p<10^{-6}\right)$.

Important regions determined by support vector machine recursive feature elimination feature selection

Table 5 lists the top 10\% ranked regions determined by the SVM-RFE feature selection method. The label abbreviations are listed in Table 6. Regarding MRI-based features, the left hippocampus ranked first in AAL, and the left parahippocampal gyrus ranked first in LPBA40. For the PET-based features, the left inferior temporal gyrus ranked first in AAL followed by the right hippocampus and right precentral gyrus, whereas the middle temporal 
gyrus ranked first in LPBA40 followed by the left superior occipital gyrus and the right inferior frontal gyrus. When combining MRI and PET feature vectors, the PET-based regions generally ranked higher than did the MRI-based regions except for the combination of MRILPBA40 and PET-AAL.

As shown in Fig. 6, we mapped the regions listed in Table 5 to the surface of the brain. Compared with MRI-based features (upper left) in which the left medial temporal regions in both atlases ranked highest, PET-based features (upper right) selected the lateral temporal regions, not the medial temporal regions. When combining MRI and PET data (bottom), the right superior frontal gyrus in the AAL ranked high among the PET-based regions.

\section{Discussion}

The principal aim of this study was to assess the effects of imaging modalities and brain atlases for feature extraction on prediction of conversion from MCI to AD. The results of this study indicate that preferable brain atlases for feature extraction can differ on the basis of the imaging modality used for unimodal features and for multimodal features and that combining multimodal imaging biomarkers improved the ability to predict conversion from MCI to AD. We believe what is practically important for this prediction is the choice of biomarkers (features) to be used and how to combine these biomarkers (Frisoni et al., 2013).

Our results suggest not only that multimodal features provide better performance for prediction than unimodal features but also that FDG-PET can be superior to MRI for early detection of $\mathrm{AD}$, particularly when using $\mathrm{AAL}$ for feature extraction. For example, our twoway ANOVA for comparing the effects of atlases in multimodal feature sets demonstrated 
that the effect of MRI atlas at AAL for PET was not different. That is, the AAL-based multimodal combination (MRI-AAL + PET-AAL), which contained the weakest feature set (MRI-AAL), was comparable to the better heterogeneous multimodal combination (MRILPBA40 + PET-AAL). The other combinations both comprised PET-LPBA40, which gave an AUC lower than that of PET-AAL but higher than that of MRI-AAL. One possible explanation is that FDG-PET biomarkers, particularly when extracted with AAL, can better detect the difference in temporospatial distribution patterns of synaptic loss in the brains of patients with aMCI. Besides amyloid plaques and NFTs, AD is also characterized by the loss of neurons and their synapses particularly in the cerebral cortex and hippocampus (Scheff and D. A. Price, 2006). Although amyloid plaques and NFTs are the major neuropathological hallmarks of $\mathrm{AD}$, amyloid accumulation in the brains of patients with $\mathrm{AD}$ is probably not the cause of the neurodegeneration but merely a marker of some upstream alterations that can cause neuronal and synaptic loss (Drachman, 2014). Synaptic loss and neuronal loss are the major pathological substrates of cortical atrophy (Serrano-Pozo et al., 2011). Synaptic loss is the major correlate of cognitive impairment (Terry et al., 1991) and the most reliable index of cognition in both postmortem and biopsied AD brains (DeKosky et al., 1996). In vivo ${ }^{18} \mathrm{~F}$ FDG uptake is strongly correlated with cerebral synaptic density and activity (Rocher et al., 2003). Cognitive decline is strongly associated with glucose hypometabolism in frontal and temporoparietal regions in patients with probable AD (Furst et al., 2012). Temporal and parietal glucose metabolism predict decline in global cognitive function, and medial temporal brain volumes predict memory decline in normal older people (Jagust et al., 2006). These findings and our results, therefore, support that FDG-PET biomarkers and particularly multimodal combinations with other imaging modalities, such as structural MRI, can have an important role in cognitive decline. 
From Table 1 it is clear that the converters are more impaired cognitively than non-converters, particularly verbal memory measured by WMS-R LM. We tested the prediction accuracy using these cognitive scores (Supplementary Material). WMS-R LM II alone demonstrated the highest AUC value among the cognitive feature sets, which outperformed imaging biomarkers alone as we expected (Supplementary Fig. 1). However, some combined feature sets of cognitive scores and imaging biomarkers outperformed cognitive scores alone or imaging biomarkers alone, and the performance varied depending on how to combine these measures (Supplementary Figs. 2 and 3). First, diagnosis of dementia (due to AD) is done clinically. Cognitive scores such as episodic memory measures including WMS-R LM I and II immediate and delayed recall were used for cognitive assessment to differentiate MCI from AD. Thus cognitive scores are somewhat like supervisory signals, and use of cognitive scores for prediction may not be suitable. Second, even if the imaging biomarkers bring little additional discriminative information in terms of predictive accuracy, these injury biomarkers bring additional topographic information that may help to characterize clinical phenotypes (patterns of cortical atrophy or hypometabolism) (Dubois et al., 2014). Therefore we believe that imaging biomarkers have some advantages to unravel the underlying topographic pathophysiological features compared to cognitive scores.

Regarding the effect of atlas on predictive performance, our two-way ANOVA for comparing the effects of atlas and modality in the unimodal feature sets demonstrated that the performance differed on the basis of the atlases on both MRI and PET. The major difference between AAL and LPBA40 is the number of ROIs. AAL has 116 ROIs, more than twice the number in LPBA40 (56 ROIs). In addition, AAL includes 26 finely parcellated cerebellar regions, whereas LPBA40 contains the cerebellum as only one large region. These 
differences in parcellation between these atlases may be associated with the different effects on the classification performance.

Previous studies have shown superior accuracies using other methods than atlas-based approaches. However, in view of clinical applications, for example, electrocardiogram with limited leads, methods for measurement and analysis would be better as simple as possible, as long as the performance is not too poor. Atlas-based analysis is not the best in performance compared to other methods, but it provides an accuracy of around $70 \%$. In addition, atlasbased methods may be good for clinical applications because clinical practitioners usually interpret the results in an anatomical framework. Results therefore should be given regionsof-interest (ROIs) using anatomical notation whatever features are used. Even if data-driven parcellation methods are used, we need to present the results using a reference like the MNI coordinate system. We therefore believe that it is worth using the atlas-based analysis.

Another purpose of this study was to assess the influence of SVM-RFE feature selection on the performance of MCI classification. The importance of feature selection in machine learning studies for AD prediction remains controversial (Chu et al., 2012; Cuingnet et al., 2011; Kerr et al., 2014). In this study, we found a significant main effect of SVM-RFE on the performance of MCI classification. Depending on the atlases and modalities, the influence of SVM-RFE can also differ, although there were no significant interactions between SVM-RFE and the other factors. We think that this may be because of a kind of ceiling effect, which resulted from the difficulty in binary classification of MCI into converters and nonconverters. As compared with discrimination of MCI from or AD from normal age-related deficits (Cuingnet et al., 2011), the accuracy of MCI classification is at most approximately $80 \%$ (Cuingnet et al., 2011; Misra et al., 2009; Querbes et al., 2009). This may be partly because 
of the intrinsic heterogeneity in MCI (Nettiksimmons et al., 2014) or possibly because of variations in the spatial and temporal progression of pathological changes. In addition, "nonconverters" include possible converters in principle because nonconverters may develop $\mathrm{AD}$ after 3 years of follow-up.

The optimal prediction accuracy was achieved using multiple features. For MRI it was around 25 when using LPBA40. This suggests that removing less discriminating set of features by feature selection may improve predictive accuracy. However, for PET and multimodal approaches, the optimal was without selection. These results should be compared with a recent study, where only 5 features optimize the prediction (Eskildsen et al., 2015). There could be a potential problem of overfitting the model when the number of features is greater than that of subjects. Overfitting leads to poor generalization.

We evaluated this atlas-based approach using the ADNI dataset as an independent cohort (Supplementary Material). Although atlas $\times$ modality interaction was also significant in the ADNI dataset, the results of ADNI were different from those of SEAD-J. One possible explanation is sample selection bias. The ADNI dataset and our dataset are different in including criteria (Kawashima et al., 2012), resulting in different baseline characteristics. In addition, individual genetic variations exert lasting influences on brain structures and functions associated with behavior and predisposition to disease (Hibar et al., 2015). This may also be associated with the different results.

Such sample selection bias affects both learning and evaluation (Zadrozny, 2004). Learning methods can be classified into global and local learners. Global learners, such as naive Bayes, soft margin SVM, and decision tree learners, are affected by sample selection bias, while 
local learners, such as logistic regression and hard margin SVM, are insensitive to sample selection bias. However, the evaluation step is always affected by sample selection bias.

In ADNI datasets, multimodal datasets did not significantly outperform unimodal datasets. The sample size of the ADNI subjects was 158, almost double the number of subjects SEAD$\mathrm{J}, 80$. Overfitting in the SEAD-J results for multimodal feature sets cannot be ruled out. If there is no overfitting in the ADNI results, increased-feature sizes including irrelevant features may be associated with no improvement in the performance for the ADNI multimodal feature sets.

Another difference between ADNI and SEAD-J was the effect of feature selection. In the case of ADNI, the effect of SVM-RFE on prediction performance was remarkable compared to SEAD-J, particularly when using unimodal datasets. Difference in sample size between these datasets may also be associated with the difference in the difference in the effect of feature selection. Regarding feature selection in small sample size data, the lack of relation between the errors of the best and selected feature sets was observed more evidently in smaller sample sizes than for larger sample sizes (Sima and Dougherty, 2006). In addition, SVM-RFE is generally sensitive to noise and outliers (Niijima and Kuhara, 2006) when it is applied to small sample size data. However, increase in sample size may result in a more heterogeneous sample, and this heterogeneity may make it difficult to predict conversion. Stratification into more homogeneous subgroups may help us predict AD conversion and understand underlying pathophysiological mechanisms.

We believe that SVM-RFE feature selection is useful not only for reducing the dimension of the feature space to avoid the issue of dimensionality and overfitting but also for finding 
valuable regions in view of the consistency of the selected regions (the left hippocampus on MRI and the inferior temporal gyrus, the precuneus, and the angular gyrus on FDG-PET) observed by using our voxel-based analysis and observed in previous studies (Chételat et al., 2005; Zhang et al., 2011). In this study, the inferior temporal gyrus and the precuneus were identified as important regions for MCI classification by using both SVM-RFE and voxelbased analysis from FDG-PET data. Scheff et al. reported synaptic loss in the inferior temporal gyrus (Scheff et al., 2011) and the precuneus (Scheff et al., 2013) in aMCI patients. According to their studies, the inferior temporal gyrus is affected during the prodromal stage of the disease (Scheff et al., 2011), whereas the precuneus does not show early changes in synaptic decline during the progression of AD (Scheff et al., 2013). On the other hand, significant ${ }^{18}$ F-FDG uptake reductions in patients with very mild AD relative to that in normal controls have been found in the precuneus and other neocortical regions, including the posterior cingulate and left temporoparietal and frontal association cortex (Herholz et al., 2002). This spatiotemporal difference in local cerebral glucose metabolism is similar to the characteristic progression patterns of NFTs (Braak and Braak, 1991) and synaptic loss (Scheff and Price, 2006), which originate from the entorhinal cortex and extend through the limbic regions to the neocortex during disease progression. The inferior temporal gyrus has neural interconnections with the structures in the medial temporal cortex, particularly the parahippocampal gyrus (Suzuki and Amaral, 1994) and has an important role in verbal fluency (Scheff et al., 2011). Regional cerebral glucose metabolism in the left inferior temporal region in the brains of patients with mild $\mathrm{AD}$ correlates consistently with verbal semantic memory measures (Hirono et al., 2001). A meta-analysis showed that verbal fluency, particularly semantic fluency rather than phonemic fluency, was significantly more impaired than measures of verbal intelligence and psychomotor speed, and episodic memory appeared to be most disrupted by AD (Henry et al., 2004). These findings are consistent with the 
significant differences in WMS-R-LM in the present study. As mentioned previously (Ota et al., 2014), most of the participants in this study were subclassified as having late MCI, which is a high risk group for $\mathrm{AD}$ (Jessen et al., 2014) and defined by the education-adjusted ranges of the WMS-R-LM II score (Aisen et al., 2010). Decline in verbal functions may be associated with the left-dominant laterality in the significant regions in our voxel-based analysis. These findings raise the possibility that SVM-RFE feature selection could detect temporally different underlying pathological progressions. If so, these findings and our results suggest that glucose hypometabolism in the inferior temporal gyrus might be an early biomarker for discriminating converters from nonconverters. In addition, the left hippocampus (AAL 37) on MRI is a robust biomarker for MCI binary classification as a single feature (Ota et al., 2014). Stratification of MCI into subtypes with combined specific features could provide better diagnostic ability or more detailed information on AD/MCI pathophysiology. To achieve further evidence, evaluation by using other datasets is desired in the future. In addition, it would be useful to apply atlas-based analysis to other imaging biomarkers, such as white matter hyperintensities (Nettiksimmons et al., 2014; Provenzano et al., 2013).

Our validation set up was rather complex. Validation such as 10 -fold cross-validation would be easier to be implemented than LOOCV. However, several previous studies used LOOCV (Eskildsen et al., 2013, 2015). A previous study mentioned that 10 -fold cross-validation and LOOCV is not significantly different when the number of features is 10 to 100 (Chu et al., 2012, Supplementary data). We therefore consider that use of LOOCV in this study is not a major problem. 
A possible limitation of our study is the MRI acquisition on multiple scanners including $1.5 \mathrm{~T}$ and $3 \mathrm{~T}$. The numbers of images for 9 sites (A-I) were $3,15,15,5,7,16,7,2$, and 10 , respectively ( $\chi^{2}$ test, $p=0.001$ ). There could be potential biases. Although the influence of multi-slice imaging on contrast-to-noise ratio between gray and white matter is significantly larger at $3 \mathrm{~T}$ than at $1.5 \mathrm{~T}$ (Fushimi et al., 2007), the influence may be limited because only 3 participants (4\%) were scanned on $3 \mathrm{~T}$ in this study.

Atlas-based feature extraction is methodologically simple and has a much lower computational cost than voxel-based adaptive approaches. In addition, atlas-based analysis can provide regional information within each ROI in the brains of subjects that can readily be interpreted by clinicians. In view of possible clinical applications, our results suggest that the use of AAL may be recommended for feature extraction from PET or multimodal biomarkers.

\section{Conclusions}

Our study demonstrated that imaging modalities and brain atlases for feature extraction affect prediction and interact with each other. In addition, SVM-RFE feature selection can itself improve the performance of classification. Regions selected by SVM-RFE were generally consistent with those selected in previous studies and with our results from group comparisons. Besides atlas-based analysis, other techniques for feature extraction include data-driven parcellation methods (Fan et al., 2007) and dimension reduction by factor analysis (Desikan et al., 2010). Data-driven approaches can well reflect the underlying structure of the data, whereas they are usually computationally expensive and the resulting output tends to be complicated and have interpretational difficulties. Dimension reduction by factor analysis can also learn intrinsic data structure without the need for supervisory signals 
resulting in good generalization, whereas it is not always possible to improve the predictive accuracy. Regarding features for classification, the Ugly Duckling theorem (Watanabe, 1969) indicates that an MCI nonconverter is as similar to an MCI converter as to another nonconverter. Selection of features for classification is arbitrary, and based on subjective criteria that depend on the purpose or the task. If feature selection for $\mathrm{AD}$ prediction is done based on an algorithm, no free lunch theorems (Wolpert, 1996) may also hold true. This implies that prior domain knowledge may be important for AD prediction. Although the sample size was limited, we consider atlas-based analysis to be a useful tool for finding promising biomarkers because of its simplicity and ease of interpreting the results. In the future, the combination of atlas-based analysis with unsupervised feature representation learning methods may be a useful tool for finding novel biomarkers. Longitudinal analysis using our method will be useful for further investigation of MCI classification.

\section{Acknowledgments}

This work was supported by a Health Labor Sciences Research Grant from the Ministry of Health, Labor, and Welfare of Japan (H17-Tyojyu-023) and the Research Funding for Longevity Sciences from the National Center for Geriatrics and Gerontology, Japan (20-1). This work was partly supported by the following: Grant-in-Aid for Young Scientists (B) 24791296 from the Japan Society for the Promotion of Science (JSPS) (to N.O.) and Development of BMI Technologies for Clinical Application under the Strategic Research Program for Brain Sciences by the Ministry of Education, Culture, Sports, Science, and Technology of Japan (to N.O.). The authors appreciate the efforts and contributions of those who engaged in the subjects' care and the collection of FDG-PET and MRI images and clinical reports. 


\section{References}

Aisen PS, Petersen RC, Donohue MC, Gamst A, Raman R, Thomas RG, et al. Clinical core of the Alzheimer's disease neuroimaging initiative: progress and plans. Alzheimers Dement 2010;6:239-246.

Albert MS, DeKosky ST, Dickson D, Dubois B, Feldman HH, Fox NC, et al. The diagnosis of mild cognitive impairment due to Alzheimer's disease: recommendations from the National Institute on Aging-Alzheimer's Association workgroups on diagnostic guidelines for Alzheimer's disease. Alzheimers Dement 2011;7:270-279.

Alzheimer's Association. 2013 Alzheimer's disease facts and figures. Alzheimers Dement $2013 ; 9: 208-245$.

Bohland JW, Bokil H, Allen CB, Mitra PP. The brain atlas concordance problem: quantitative comparison of anatomical parcellations. PLoS ONE 2009;4:e7200.

Braak H, Braak E. Neuropathological stageing of Alzheimer-related changes. Acta Neuropathol. 1991;82:239-259.

Breiman L. Bagging predictors. Mach Learn 1996;24:123-140.

Chang C-C, Lin C-J. LIBSVM: a library for support vector machines. ACM Trans Intell Syst Technol 2011;2:27:1-27:27.

Chao LL, Buckley ST, Kornak J, Schuff N, Madison C, Yaffe K, et al. ASL perfusion MRI predicts cognitive decline and conversion from MCI to dementia. Alzheimer Dis Assoc Disord 2010;24:19-27.

Chételat G, Landeau B, Eustache F, Mézenge F, Viader F, la Sayette de V, et al. Using voxelbased morphometry to map the structural changes associated with rapid conversion in MCI: a longitudinal MRI study. Neuroimage 2005;27:934-946. 
Chu C, Hsu A-L, Chou K-H, Bandettini P, Lin C, Alzheimer's disease neuroimaging initiative. Does feature selection improve classification accuracy? Impact of sample size and feature selection on classification using anatomical magnetic resonance images. Neuroimage 2012;60:59-70.

Cohen J. Eta-squared and partial eta-squared in fixed factor Anova designs. Educ Psychol Meas 1973;33:107-112.

Cohen J. A power primer. Psychol Bull 1992;112:155-159.

Cuingnet R, Gerardin E, Tessieras J, Auzias G, Lehéricy S, Habert M-O, et al. Automatic classification of patients with Alzheimer's disease from structural MRI: A comparison of ten methods using the ADNI database. Neuroimage 2011;56:766-781.

DeKosky ST, Scheff SW, Styren SD. Structural correlates of cognition in dementia: quantification and assessment of synapse change. Neurodegeneration 1996;5:417-421.

Desikan RS, Cabral HJ, Settecase F, Hess CP, Dillon WP, Glastonbury CM, et al. Automated MRI measures predict progression to Alzheimer's disease. Neurobiol Aging 2010;31:1364-1374.

Dimitriadou E, Hornik K, Leisch F, Meyer D. Misc functions of the department of statistics (e1071), TU Wien. Available from http://cran.rproject.org/web/packages/e1071/index.html; 2005.

Dosenbach NUF, Nardos B, Cohen AL, Fair DA, Power JD, Church JA, et al. Prediction of individual brain maturity using fMRI. Science 2010;329:1358-1361.

Drachman DA. The amyloid hypothesis, time to move on: Amyloid is the downstream result, not cause, of Alzheimer's disease. Alzheimers Dement 2014;10:372-380.

Duan K-B, Rajapakse JC, Wang H, Azuaje F. Multiple SVM-RFE for gene selection in cancer classification with expression data. IEEE Trans Nanobiosc 2005;4:228-234. 
Dubois B, Feldman HH, Jacova C, Hampel H, Molinuevo JL, Blennow K, et al. Advancing research diagnostic criteria for Alzheimer's disease: the IWG-2 criteria. Lancet Neurol 2014;13:614-629.

Duda RO, Hart PE, Stork DG. Pattern Classification. Second Edition. New York: John Wiley \& Sons, Inc; 2001.

Dukart J, Mueller K, Horstmann A, Vogt B, Frisch S, Barthel H, et al. Differential effects of global and cerebellar normalization on detection and differentiation of dementia in FDG-PET studies. Neuroimage 2010;49:1490-1495.

Eskildsen SF, Coupé P, García-Lorenzo D, Fonov V, Pruessner JC, Collins DL, et al. Prediction of Alzheimer's disease in subjects with mild cognitive impairment from the ADNI cohort using patterns of cortical thinning. Neuroimage 2013;65:511-521.

Eskildsen SF, Coupé P, Fonov VS, Pruessner JC, Collins DL, Alzheimer's disease neuroimaging initiative. Structural imaging biomarkers of Alzheimer's disease: predicting disease progression. Neurobiol Aging 2015;36 Suppl 1:S23-31.

Fan Y, Shen D, Gur RC, Gur RE, Davatzikos C. COMPARE: classification of morphological patterns using adaptive regional elements. IEEE Trans Med Imaging 2007;26:93-105.

Fawcett T. ROC graphs: notes and practical considerations for researchers. Mach Learn 2004:1-38.

Folstein MF, Folstein SE, McHugh PR. 'Mini-mental state'. A practical method for grading the cognitive state of patients for the clinician. J Psychiatr Res 1975;12:189-198.

Fox J, Weisberg S. Car: companion to applied regression. Available at http://cran.rproject.org/web/packages/car/index.html; 2009.

Frisoni GB, Bocchetta M, Chételat G, Rabinovici GD, de Leon MJ, Kaye J, et al. Imaging markers for Alzheimer disease: which vs how. Neurology 2013;81:487-500. 
Furst AJ, Rabinovici GD, Rostomian AH, Steed T, Alkalay A, Racine C, et al. Cognition, glucose metabolism and amyloid burden in Alzheimer's disease. Neurobiol Aging 2012;33:215-225.

Fushimi Y, Miki Y, Urayama S-I, Okada T, Mori N, Hanakawa T, et al. Gray matter-white matter contrast on spin-echo T1-weighted images at 3-T and 1.5-T: a quantitative comparison study. Eur Radiol 2007;17:2921-2925.

Giacobini E, Gold G. Alzheimer disease therapy—moving from amyloid- $\beta$ to tau. Nat Rev Neurol 2013;9:677-686.

Guyon I, Weston J, Barnhill S, Vapnik V. Gene selection for cancer classification using support vector machines. Mach Learn 2002;46:389-422.

Henry JD, Crawford JR, Phillips LH. Verbal fluency performance in dementia of the Alzheimer's type: a meta-analysis. Neuropsychologia 2004;42:1212-1222.

Herholz K, Salmon E, Perani D, Baron J-C, Holthoff V, Frölich L, et al. Discrimination between Alzheimer dementia and controls by automated analysis of multicenter FDG PET. Neuroimage 2002;17:302-316.

Hibar DP, Stein JL, Renteria ME, Arias-Vasquez A, Desrivières S, Jahanshad N, et al. Common genetic variants influence human subcortical brain structures. Nature $2015 ; 520: 224-229$.

Hirono N, Mori E, Ishii K, Imamura T, Tanimukai S, Kazui H, et al. Neuronal substrates for semantic memory: a positron emission tomography study in Alzheimer's disease. Dement Geriatr Cogn Disord 2001;12:15-21.

Homma A, Fukuzawa K, Tsukada Y, Ishii T, Hasegawa K, Mohs RC. Development of a Japanese version of Alzheimer's Disease Assessment Scale (ADAS). Jpn J Geriatr Psychiary 1992;3:647-655. 
Huang J, Ling CX. Using AUC and accuracy in evaluating learning algorithms. Knowl Data Eng, IEEE Trans 2005;17:299-310.

Hyman BT, Phelps CH, Beach TG, Bigio EH, Cairns NJ, Carrillo MC, et al. National Institute on Aging-Alzheimer's Association guidelines for the neuropathologic assessment of Alzheimer's disease. Alzheimers Dement 2012;8:1-13.

Ito K, Mori E, Fukuyama H, Ishii K, Washimi Y, Asada T, et al. Prediction of outcomes in MCI with (123)I-IMP-CBF SPECT: a multicenter prospective cohort study. Ann Nucl Med 2013;27:898-906.

Ito K, Fukuyama H, Senda M, Ishii K, Maeda K, Yamamoto Y, et al. Prediction of outcomes in mild cognitive impairment by using 18F-FDG-PET: a multicenter study. $\mathrm{J}$ Alzheimers Dis 2015;45:543-552.

Jack CR, Albert MS, Knopman DS, McKhann GM, Sperling RA, Carrillo MC, et al. Introduction to the recommendations from the National Institute on AgingAlzheimer's Association workgroups on diagnostic guidelines for Alzheimer's disease. Alzheimers Dement 2011;7:257-262.

Jack CR, Holtzman DM. Biomarker modeling of Alzheimer's disease. Neuron 2013;80:13471358.

Jack CR, Knopman DS, Jagust WJ, Shaw LM, Aisen PS, Weiner MW, et al. Hypothetical model of dynamic biomarkers of the Alzheimer's pathological cascade. Lancet Neurol 2010;9:119-128.

Jagust W, Gitcho A, Sun F, Kuczynski B, Mungas D, Haan M. Brain imaging evidence of preclinical Alzheimer's disease in normal aging. Ann Neurol 2006;59:673-681.

Jessen F, Wolfsgruber S, Wiese B, Bickel H, Mösch E, Kaduszkiewicz H, et al. AD dementia risk in late MCI, in early MCI, and in subjective memory impairment. Alzheimers Dement 2014;10:76-83. 
Karow DS, McEvoy LK, Fennema-Notestine C, Hagler DJ, Jennings RG, Brewer JB, et al. Relative capability of MR imaging and FDG PET to depict changes associated with prodromal and early Alzheimer disease. Radiology 2010;256:932-942.

Kawashima S, Ito K, Kato T, SEAD-J study group. Inclusion criteria provide heterogeneity in baseline profiles of patients with mild cognitive impairment: comparison of two prospective cohort studies. Br Med J Open 2012;2:e000773.

Kerr WT, Douglas PK, Anderson A, Cohen MS. The utility of data-driven feature selection: re: Chu et al. 2012. Neuroimage 2014;84:1107-1110.

Kurth F, Luders E, Gaser C. VBM8-Toolbox Manual [Internet]. 2010. Available from: http://dbm.neuro.uni-jena.de/vbm

Li H-J, Hou X-H, Liu H-H, Yue C-L, He Y, Zuo X-N. Toward systems neuroscience in mild cognitive impairment and Alzheimer's disease: a meta-analysis of 75 fMRI studies. Hum Brain Mapp 2015;36:1217-1232.

Langsrud Ø. ANOVA for unbalanced data: use type II instead of type III sums of squares. Stat Comput 2003;13:163-167.

McKhann GM, Knopman DS, Chertkow H, Hyman BT, Jack CR Jr, Kawas CH, et al. The diagnosis of dementia due to Alzheimer's disease: recommendations from the National Institute on Aging-Alzheimer's Association workgroups on diagnostic guidelines for Alzheimer's disease. Alzheimers Dement 2011;7:263-269.

Metz CE. Basic principles of ROC analysis. Semin Nucl Med 1978;8:283-298.

Mevel K, Desgranges B, Baron J-C, Landeau B, la Sayette de V, Viader F, et al. Detecting hippocampal hypometabolism in mild cognitive impairment using automatic voxelbased approaches. Neuroimage 2007;37:18-25. 
Misra C, Fan Y, Davatzikos C. Baseline and longitudinal patterns of brain atrophy in MCI patients, and their use in prediction of short-term conversion to AD: results from ADNI. Neuroimage 2009;44:1415-1422.

Mosconi L, Sorbi S, De Leon MJ, Li Y, Nacmias B, Myoung PS, et al. Hypometabolism exceeds atrophy in presymptomatic early-onset familial Alzheimer's disease. J Nucl Med 2006;47:1778-1786.

Mufson EJ, Binder L, Counts SE, DeKosky ST, de Toledo-Morrell L, Ginsberg SD, et al. Mild cognitive impairment: pathology and mechanisms. Acta Neuropathol. 2012;123:13-30.

Nettiksimmons J, DeCarli C, Landau S, Beckett L, Alzheimer's disease neuroimaging initiative. Biological heterogeneity in ADNI amnestic mild cognitive impairment. Alzheimers Dement 2014;10:511-521.e1.

Niijima S, Kuhara S. Recursive gene selection based on maximum margin criterion: a comparison with SVM-RFE. BMC Bioinform 2006;7:543.

Nyunt MSZ, Fones C, Niti M, Ng T-P. Criterion-based validity and reliability of the Geriatric Depression Screening Scale (GDS-15) in a large validation sample of communityliving Asian older adults. Aging Ment Health 2009;13:376-382.

Oishi K, Akhter K, Mielke M, Ceritoglu C, Zhang J, Jiang H, et al. Multi-modal MRI analysis with disease-specific spatial filtering: initial testing to predict mild cognitive impairment patients who convert to Alzheimer's disease. Front Neurol 2011;2:54.

Ota K, Oishi N, Ito K, Fukuyama H, SEAD-J study group. A comparison of three brain atlases for MCI prediction. J Neurosci Methods 2014;221:139-150.

Petersen RC, Doody R, Kurz A, Mohs RC, Morris JC, Rabins PV, et al. Current concepts in mild cognitive impairment. Arch Neurol 2001;58:1985-1992. 
Petersen RC, Smith GE, Waring SC, Ivnik RJ, Tangalos EG, Kokmen E. Mild cognitive impairment: clinical characterization and outcome. Arch Neurol 1999;56:303-308.

Price JC. Molecular brain imaging in the multimodality era. J Cereb Blood Flow Metab 2012;32:1377-1392.

Provenzano FA, Muraskin J, Tosto G, Narkhede A, Wasserman BT, Griffith EY, et al. White matter hyperintensities and cerebral amyloidosis: necessary and sufficient for clinical expression of Alzheimer disease? J Am Med Assoc Neurol 2013;70:455-461.

Querbes O, Aubry F, Pariente J, Lotterie JA, Demonet JF, Duret V, et al. Early diagnosis of Alzheimer's disease using cortical thickness: impact of cognitive reserve. Brain 2009;132:2036-2047.

R Development Core Team. R: A language and environment for statistical computing. Vienna: R Foundation for Statistical Computing; 2013.

Robin X, Turck N, Hainard A, Tiberti N, Lisacek F, Sanchez J-C, et al. pROC: an opensource package for $\mathrm{R}$ and $\mathrm{S}+$ to analyze and compare ROC curves. BMC Bioinform $2011 ; 12: 77$.

Rocher AB, Chapon F, Blaizot X, Baron J-C, Chavoix C. Resting-state brain glucose utilization as measured by PET is directly related to regional synaptophysin levels: a study in baboons. Neuroimage 2003;20:1894-1898.

Scheff SW, Price DA, Schmitt FA, Mufson EJ. Hippocampal synaptic loss in early Alzheimer's disease and mild cognitive impairment. Neurobiol Aging 2006;27:13721384.

Scheff SW, Price DA, Schmitt FA, Roberts KN, Ikonomovic MD, Mufson EJ. Synapse stability in the precuneus early in the progression of Alzheimer's disease. $\mathrm{J}$ Alzheimers Dis 2013;35:599-609. 
Scheff SW, Price DA, Schmitt FA, Scheff MA, Mufson EJ. Synaptic loss in the inferior temporal gyrus in mild cognitive impairment and Alzheimer's disease. J Alzheimers Dis $2011 ; 24: 547-557$.

Scheff SW, Price DA. Alzheimer's disease-related alterations in synaptic density: neocortex and hippocampus. Journal of Alzheimer's disease : JAD 2006;9:101-115.

Serrano-Pozo A, Frosch MP, Masliah E, Hyman BT. Neuropathological alterations in Alzheimer disease. Cold Spring Harb Perspect Med 2011;1:a006189.

Shattuck DW, Mirza M, Adisetiyo V, Hojatkashani C, Salamon G, Narr KL, et al. Construction of a 3D probabilistic atlas of human cortical structures. Neuroimage 2008;39:1064-1080.

Shokouhi S, Claassen D, Kang H, Ding Z, Rogers B, Mishra A, et al. Longitudinal progression of cognitive decline correlates with changes in the spatial pattern of brain 18F-FDG PET. J Nucl Med 2013;54:1564-1569.

Sima C, Dougherty ER. What should be expected from feature selection in small-sample settings. Bioinformatics 2006;22:2430-2436.

Sperling RA, Aisen PS, Beckett LA, Bennett DA, Craft S, Fagan AM, et al. Toward defining the preclinical stages of Alzheimer's disease: recommendations from the National Institute on Aging-Alzheimer's Association workgroups on diagnostic guidelines for Alzheimer's disease. Alzheimers Dement 2011;7:280-292.

Sullivan K. Estimates of interrater reliability for the logical memory subtest of the Wechsler memory scale-revised. J Clin Exp Neuropsychol 1996;18:707-712.

Suzuki WA, Amaral DG. Perirhinal and parahippocampal cortices of the macaque monkey: cortical afferents. J Comp Neurol 1994;350:497-533. 
Terry RD, Masliah E, Salmon DP, Butters N, DeTeresa R, Hill R, et al. Physical basis of cognitive alterations in Alzheimer's disease: synapse loss is the major correlate of cognitive impairment. Ann Neurol 1991;30:572-580.

Tzourio-Mazoyer N, Landeau B, Papathanassiou D, Crivello F, Etard O, Delcroix N, et al. Automated anatomical labeling of activations in SPM using a macroscopic anatomical parcellation of the MNI MRI single-subject brain. Neuroimage 2002;15:273-289.

Valentini G, Muselli M, Ruffino F. Cancer recognition with bagged ensembles of support vector machines. Neurocomputing 2004;56:461-466.

Vapnik VN. Statistical learning theory. New York: Wiley; 1998.

Watanabe S. Knowing and guessing: A quantitative study of inference and information. New York: Wiley; 1969.

Wolpert DH. The existence of a priori distinctions between learning algorithms. Neural Comput 1996;8:1391-1420.

Xia M, Wang J, He Y. BrainNet viewer: a network visualization tool for human brain connectomics. PLoS One 2013;8:e68910.

Yesavage JA, Brink TL, Rose TL, Lum O, Huang V, Adey M, et al. Development and validation of a geriatric depression screening scale: a preliminary report. J Psychiatr Res 1982;17:37-49.

Young J, Modat M, Cardoso MJ, Mendelson A, Cash D, Ourselin S, et al. Accurate multimodal probabilistic prediction of conversion to Alzheimer's disease in patients with mild cognitive impairment. Neuroimage Clin 2013;2:735-745.

Zadrozny B. Learning and evaluating classifiers under sample selection bias. In: Proceedings of the twenty-first international conference on machine learning 2004:903-910. Association for Computing Machinery; New York. 
Zhang D, Wang Y, Zhou L, Yuan H, Shen D. Multimodal classification of Alzheimer's disease and mild cognitive impairment. Neuroimage 2011;55:856-867.

Table 1 Baseline characteristics of 80 participants with mild cognitive impairment (MCI).

\begin{tabular}{|c|c|c|c|c|}
\hline Characteristic & $\begin{array}{l}\text { Converters } \\
(\mathrm{n}=40)\end{array}$ & $\begin{array}{l}\text { Nonconverters } \\
\qquad(\mathrm{n}=40)\end{array}$ & $p$-value & $\begin{array}{l}\text { Effect } \\
\text { size }(r)\end{array}$ \\
\hline Demographics & & & & \\
\hline Mean age, $\mathrm{y} \pm \mathrm{SD}$ & $71.4 \pm 6.7$ & $70.5 \pm 6.7$ & 0.55 & 0.068 \\
\hline Male, n (\%) & $20(50)$ & $18(45)$ & $0.82^{\dagger}$ & $0.025^{*}$ \\
\hline Education, $\mathrm{y} \pm \mathrm{SD}$ & $12.3 \pm 3.3$ & $11.8 \pm 3.1$ & 0.46 & 0.084 \\
\hline $\begin{array}{l}\text { Neuropsychological scores, } \\
\text { mean } \pm \text { SD }\end{array}$ & & & & \\
\hline $\begin{array}{l}\text { WMS-R LM I (immediate } \\
\text { recall) }\end{array}$ & $6.6 \pm 3.3$ & $9.6 \pm 3.1$ & $<10^{-4} * * *$ & 0.42 \\
\hline $\begin{array}{l}\text { WMS-R LM II (delayed } \\
\text { recall) }\end{array}$ & $1.7 \pm 2.2$ & $4.5 \pm 2.9$ & $<10^{-5} * * *$ & 0.48 \\
\hline MMSE & $25.6 \pm 1.7$ & $27.1 \pm 2.0$ & $0.0009 * * *$ & 0.36 \\
\hline ADAS-J cog & $10.0 \pm 4.7$ & $7.7 \pm 4.5$ & $0.025 *$ & 0.25 \\
\hline GDS & $4.8 \pm 2.3$ & $3.5 \pm 1.8$ & $0.006 * *$ & 0.30 \\
\hline
\end{tabular}

Converters developed Alzheimer's disease within 3 years after inclusion. SD, standard deviation; WMS-R LM, Wechsler Memory Scale-Revised Logical memory; MMSE, MiniMental State Examination; ADAS-J cog, Alzheimer's Disease Assessment Scale-Cognitive Subscale, Japanese version; GDS, Geriatric Depression Scale. 
Student's $t$-test unless otherwise indicated, ${ }^{*} p<0.05,{ }^{* *} p<0.01,{ }^{*} * * p<0.001$.

${ }^{\dagger} \chi^{2}$ test.

$\$ \mathrm{w}$.

Table 2 Regions of significant brain gray matter loss on magnetic resonance imaging and significant brain glucose hypometabolism on fluorodeoxyglucose positron emission tomography in converters relative to those in nonconverters.

MNI coordinate

Region

\begin{tabular}{llll}
\hline$x$ & $y$ & $z$ & Peak-level T
\end{tabular} Cluster size

MRI

Left parahippocampal gyrus

$\begin{array}{lll}-27 & -44 & -3\end{array}$

4.65

$1146 *$

Left hippocampus

$\begin{array}{lll}-24 & -34 & -6\end{array}$

4.04

FDG-PET

$\begin{array}{lrrrrr} & & & \\ \text { Left precuneus } & -6 & -63 & 27 & 4.87 & * * * \\ \text { Right precuneus } & 8 & -63 & 33 & 4.37 & \\ \text { Left inferior temporal gyrus } & -56 & -31 & -23 & 4.62 & 1310 * \\ \text { Left middle temporal gyrus } & -57 & -40 & -11 & 4.08 & \\ \text { Left angular gyrus } & -46 & -57 & 30 & 4.40 & 1163 *\end{array}$

MNI, Montreal Neurological Institute; MRI, magnetic resonance imaging; FDG-PET, fluorodeoxyglucose positron emission tomography

* Uncorrected $p<0.001$, cluster-level $p<0.05$ (corrected for multiple comparisons); uncorrected $p<0.001$, cluster-level $p<0.001$ (corrected for multiple comparisons). 
Table 3 The mean area under the curve and number of features for each feature set for a three-way ANOVA design.

R: RFE

R1: RFE-

R2: RFE+

Number of Mean $\mathrm{AUC}_{\mathrm{RFE}-}\left(95 \%\right.$ Number of Mean $\mathrm{AUC}_{\mathrm{RFE}}(95 \%$

A: Atlas (combination)

features

CI)

features

CI)

A1: MRI-AAL + PET-AAL

M: Modality

M1: MRI $\quad 116 \quad 0.655(0.649-0.660) \quad 112 \quad 0.655(0.650-0.660)$

M2: PET $\quad 116 \quad 0.709(0.705-0.711) \quad 111 \quad 0.712(0.707-0.717)$

M3: MRI + PET $232 \quad 0.747(0.743-0.750) \quad 229 \quad 0.748(0.744-0.751)$

A2: MRI-AAL + PET-LPBA40

$\begin{array}{lccccc}\text { MRI } & 116 & 0.655(0.649-0.660) & 112 & 0.655(0.650-0.660) \\ \text { PET } & 56 & 0.661(0.656-0.665) & 12 & 0.671(0.657-0.686) \\ \text { MRI + PET } & 172 & 0.680(0.678-0.683) & 7 & 0.688(0.676-0.699)\end{array}$

A3: MRI-LPBA40 + PET-AAL

$\begin{array}{lrrrrr}\text { MRI } & 56 & 0.705(0.702-0.709) & 25 & 0.719(0.711-0.727) \\ \text { PET } & 116 & 0.709(0.705-0.712) & 111 & 0.712(0.707-0.717) \\ & 172 & 0.748(0.744-0.753) & 168 & 0.750(0.746-0.754)\end{array}$

A4: MRI-LPBA40 + PET-LPBA40

MRI

56

$0.705(0.702-0.709) \quad 25$

$0.719(0.711-0.727)$ 


\begin{tabular}{|c|c|c|c|c|}
\hline PET & 56 & $0.661(0.656-0.665)$ & 12 & $0.671(0.657-0.686)$ \\
\hline$[\mathrm{RI}+]$ & 11 & $.725(0.722-0.728)$ & 110 & $0.727(0.722-0.7$ \\
\hline
\end{tabular}

AUC, area under the curve; ANOVA, analysis of variance; RFE, recursive feature elimination; CI, confidence interval; SD, standard deviation; MRI, magnetic resonance imaging; PET, positron emission tomography; AAL, Automated Anatomical Labeling; LPBA40, LONI Probabilistic Brain Atlas.

Table 4 Three-way ANOVA summary table.

\begin{tabular}{lcccr}
\hline \multicolumn{1}{c}{ Source } & $(d f) F$ & $p$ & & \multicolumn{1}{c}{$\eta^{2}$} \\
\hline RFE & $(1,456)=20.1$ & $<10^{-5}$ & $* * *$ & 0.0065 \\
Atlas & $(3,456)=333.0$ & $<10^{-15}$ & $* * *$ & 0.32 \\
Modality & $(2,456)=466.3$ & $<10^{-15}$ & $* * *$ & 0.30 \\
RFE $\times$ Atlas & $(3,456)=1.3$ & 0.26 & 0.0013 \\
RFE $\times$ modality & $(2,456)=1.1$ & 0.33 & 0.0007 \\
Atlas $\times$ modality & $(6,456)=111.7$ & $<10^{-15}$ & $* * *$ & 0.22 \\
RFE $\times$ atlas $\times$ modality & $(6,456)=1.5$ & 0.19 &
\end{tabular}

Atlas at modality
Atlas at MRI
$(3,456)=227.7<10^{-15}$
Atlas at PET
$(3,456)=137.4<10^{-15}$
Atlas at MRI+PET
$(3,456)=191.2<10^{-15}$

Modality at atlas

$$
\text { Modality at MRI-AAL + PET-AAL }
$$

$(2,456)=449.3<10^{-15}$ 
Modality at MRI-AAL + PET-LPBA40 $(2,456)=44.9<10^{-15} * * * \quad 0.029$

Modality at MRI-LPBA40 + PET-AAL $(2,456)=100.5<10^{-15} * * * \quad 0.065$

Modality at MRI-LPBA40 + PET-

LPBA40

$$
(2,456)=206.6<10^{-15} \quad * * * \quad 0.13
$$

ANOVA, analysis of variance; RFE, recursive feature elimination; MRI, magnetic resonance imaging; PET, positron emission tomography; AAL, Automated Anatomical Labeling;

LPBA40, LONI Probabilistic Brain Atlas; df, degrees of freedom.

$* p<0.05, * * p<0.01, * * * p<0.001$.

Table 5 Brain regions that ranked in the top $10 \%$ of features for eight feature sets according to the multiple support vector machine feature elimination.

\begin{tabular}{|c|c|c|c|c|c|c|c|c|}
\hline \multirow[b]{4}{*}{ Rank } & \multicolumn{2}{|c|}{ MRI } & \multicolumn{2}{|c|}{ PET } & \multicolumn{4}{|r|}{$\overline{\mathrm{MRI}}$} \\
\hline & MRI & MRI & PET & PET & MRI & PET & MRI & PET \\
\hline & AAL & LPBA40 & AAL & LPBA40 & AAL & AAL & LPBA40 & LPBA40 \\
\hline & Region & Region & Region & Region & Region & Region & Region & Region \\
\hline 1 & L HIP & L PHIP & L T3 & $\mathrm{L} \mathrm{T} 2$ & - & R F1 & - & R F3 \\
\hline 2 & R F2 & L PRE & R HIP & L O1 & L HIP & - & L PHIP & - \\
\hline 3 & R P2 & R F2 & R PRE & R F3 & - & $\mathrm{L} Q$ & - & $\mathrm{L} \mathrm{O} 1$ \\
\hline 4 & R F3O & R MOFG & L T1P & L PUT & - & R CBLC1 & $\mathrm{L} \mathrm{O} 3$ & - \\
\hline 5 & L PRE & $\mathrm{L} \mathrm{O} 3$ & R CBL9 & $\mathrm{L} \mathrm{O} 2$ & R IN & - & $\mathrm{R} \mathrm{O} 2$ & - \\
\hline 6 & L Q & $\mathrm{R} \mathrm{O} 2$ & VER6 & L T1 & R HIP & - & - & $\mathrm{L}$ T2 \\
\hline 7 & R RO & - & L Q & - & - & L T3 & - & L PUT \\
\hline 8 & R POST & - & R AMYG & - & L P2 & - & R MOFG & - \\
\hline 9 & R PCIN & - & L T2P & - & - & R PRE & R IN & - \\
\hline
\end{tabular}




\begin{tabular}{|c|c|c|c|c|c|c|c|c|}
\hline 10 & L O1 & - & R P2 & - & - & R PAL & L F2 & - \\
\hline 11 & L SMA & - & L T1 & - & - & L AMYG & R CIN & - \\
\hline 12 & L F2 & - & L CBLC1 & - & L RO & - & - & $\mathrm{R} P C$ \\
\hline 13 & - & - & - & - & R PCL & - & - & - \\
\hline 14 & - & - & - & - & $\mathrm{R} \mathrm{O} 3$ & - & - & - \\
\hline 15 & - & - & - & - & - & R POST & - & - \\
\hline 16 & - & - & - & - & - & L CBLC1 & - & - \\
\hline 17 & - & - & - & - & - & VER7 & - & - \\
\hline 18 & - & - & - & - & L PHIP & - & - & - \\
\hline 19 & - & - & - & - & - & L T2P & - & - \\
\hline 20 & - & - & - & - & R SMA & - & - & - \\
\hline 21 & - & - & - & - & - & R CBL9 & - & - \\
\hline 22 & - & - & - & - & - & L F1MO & - & - \\
\hline 23 & - & - & - & - & - & L T1P & - & - \\
\hline 24 & - & - & - & - & - & R OC & - & - \\
\hline
\end{tabular}

SVM-RFE, support vector machine-based recursive feature elimination; AAL, Automated Anatomical Labeling; LPBA40, LONI Probabilistic Brain Atlas. The labels for brain regions are defined in Table 6.

Table 6 Labels for brain regions defined in the two brain atlases (AAL, Automated Anatomical Labeling; LPBA40, LONI Probabilistic Brain Atlas). The labels for AAL were adapted from Tzourio-Mazoyer et al. (2002) and modified for those for LPBA40.

AAL

Brain region

LPBA40

\begin{tabular}{lllll}
\hline Brain region & Label & & Brain region & Label
\end{tabular}


Central region

Precentral gyrus

Postcentral gyrus

Rolandic operculum

Frontal lobe

Lateral surface

Superior frontal gyrus, dorsolateral

F1

Middle frontal gyrus

Inferior frontal gyrus, opercular part

Inferior frontal gyrus, triangular part

Medial surface

Superior frontal gyrus, medial

F1M

Supplementary motor area

Paracentral lobule

Orbital surface

Superior frontal gyrus, orbital part

gyrus

Superior frontal gyrus, medial orbital

Middle frontal gyrus, orbital part

$\mathrm{F} 2 \mathrm{O}$

Inferior frontal gyrus, orbital part

F3O

Gyrus rectus

GR

Gyrus rectus

GR

Olfactory cortex

OC

Temporal lobe

Lateral surface

Superior temporal gyrus

gyrus

Heschl's gyrus

HES

$\mathrm{T} 2$

Middle temporal

$\mathrm{T} 2$

Middle temporal gyrus

gyrus 
Parietal lobe

Lateral surface

P1

Superior parietal gyrus

Inferior parietal, but supramarginal and

P2

angular gyri

$$
\begin{aligned}
& \text { Angular gyrus } \\
& \text { Supramarginal gyrus }
\end{aligned}
$$

Precuneus

PQ

Occipital lobe

Lateral surface

$\mathrm{O} 1$

Superior occipital gyrus

Middle occipital gyrus

Inferior occipital gyrus

Medial and inferior surfaces

Cuneus

Calcarine fissure and surrounding cortex

Lingual gyrus

Fusiform gyrus

Limbic lobe

Temporal pole: superior temporal gyrus

Temporal pole: middle temporal gyrus

Anterior cingulate and paracingulate gyri

Median cingulate and paracingulate gyri
Q

Cuneus

Q

V1

LING

Lingual gyrus

LING

FUSI

Fusiform gyrus

FUSI
T1P

T2P

ACIN

Cingulate gyrus

CIN 


\begin{tabular}{|c|c|c|c|c|}
\hline & Posterior cingulate gyrus & PCIN & & \\
\hline & Hippocampus & HIP & Hippocampus & HIP \\
\hline & & & Parahippocampal & \\
\hline & Parahippocampal gyrus & PHIP & gyrus & PHIP \\
\hline Insula & & $\mathrm{IN}$ & Insular cortex & IN \\
\hline Subcortical & nuclei & & & \\
\hline & Amygdala & AMYG & & \\
\hline & Caudate nucleus & $\mathrm{CAU}$ & Caudate & $\mathrm{CAU}$ \\
\hline & Lenticular nucleus, putamen & PUT & Putamen & PUT \\
\hline & Lenticular nucleus, pallidum & PAL & & \\
\hline & Thalamus & THA & & \\
\hline Cerebellum & & & Cerebellum & CBL \\
\hline & Cerebellum Crus 1 & CBLC1 & & \\
\hline & Cerebellum Crus 2 & CBLC2 & & \\
\hline & Cerebellum 3 & CBL3 & & \\
\hline & Cerebellum 45 & CBL45 & & \\
\hline & Cerebellum 6 & CBL6 & & \\
\hline & Cerebellum $7 b$ & CBL7B & & \\
\hline & Cerebellum 8 & CBL8 & & \\
\hline & Cerebellum 9 & CBL9 & & \\
\hline & Cerebellum 10 & CBL10 & & \\
\hline & Vermis 12 & VER12 & & \\
\hline & Vermis 3 & VER3 & & \\
\hline & Vermis 45 & VER45 & & \\
\hline & Vermis 6 & VER6 & & \\
\hline & Vermis 7 & VER7 & & \\
\hline & Vermis 8 & VER8 & & \\
\hline & Vermis 9 & VER9 & & \\
\hline & Vermis 10 & VER10 & & \\
\hline & & & Brainstem & BS \\
\hline
\end{tabular}




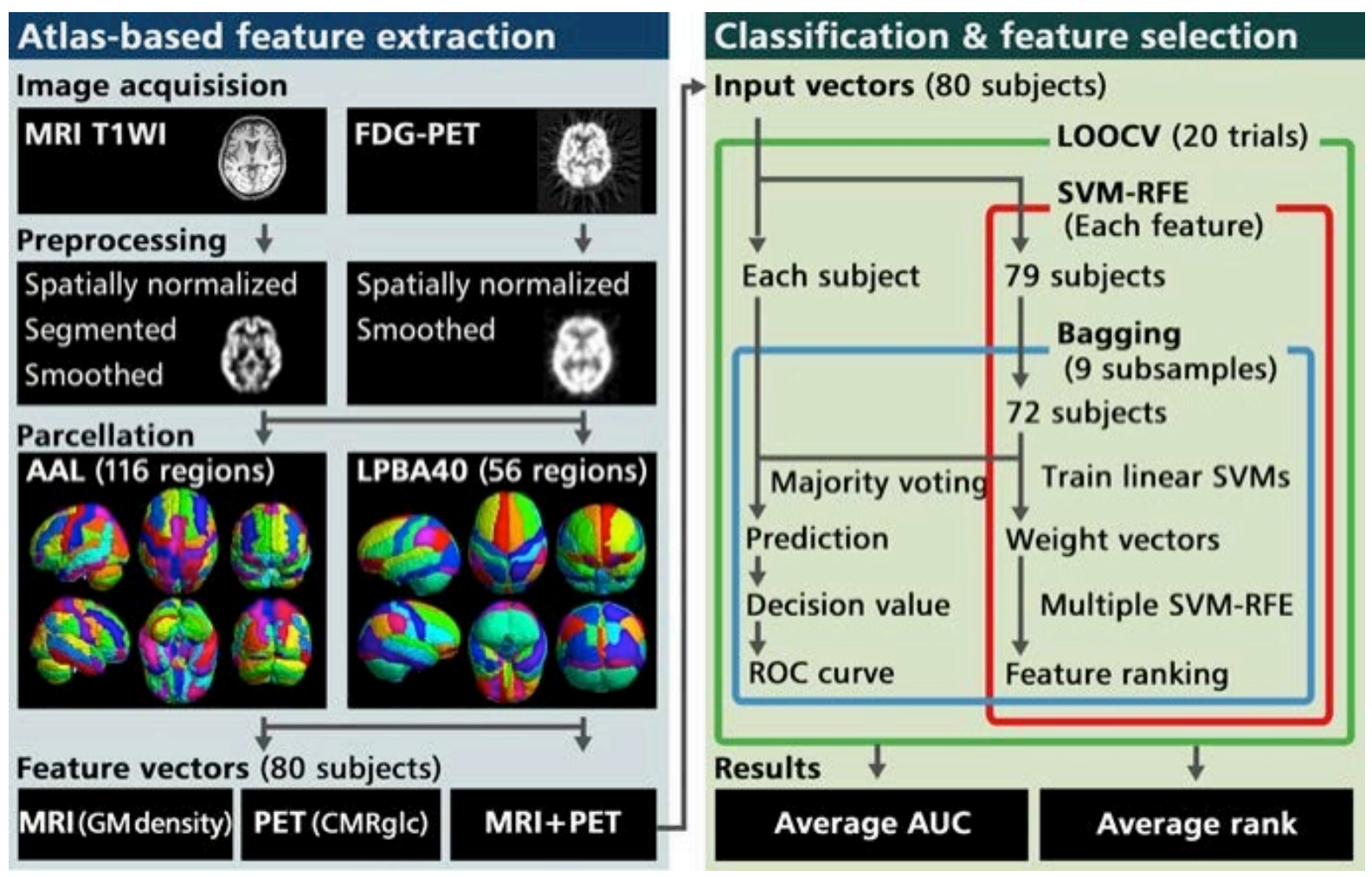

Fig. 1 Flow diagram representing steps for feature extraction, classification, and feature selection processes. MRI T1WI, magnetic resonance imaging T1-weighted image; FDG-PET, fluorodeoxyglucose positron emission tomography; AAL, Automated Anatomical Labeling; LPBA40, LONI Probabilistic Brain Atlas; GM, Gray matter; CMRglc, cerebral metabolic rate of glucose; LOOCV, leave-one-out cross-validation; SVM, Support vector machine; SVM-RFE, Support vector machine recursive feature elimination; ROC, Receiver operating characteristic; MRI, magnetic resonance imaging; PET, positron emission tomography; AUC, Area under the ROC curve. 

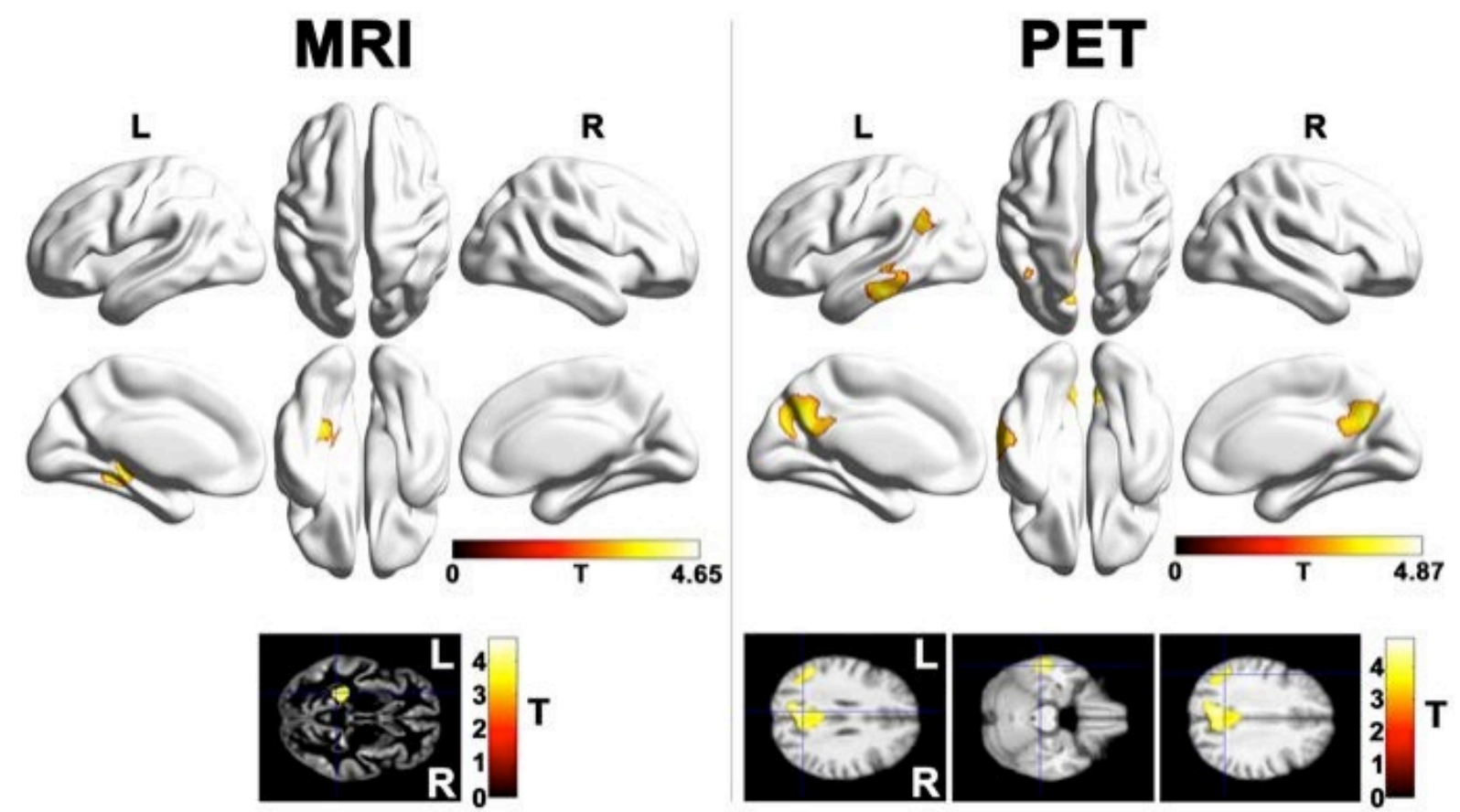

Fig. 2 Clusters of significant brain gray matter loss on magnetic resonance imaging (MRI) and significant brain glucose hypometabolism on fluorodeoxyglucose positron emission tomography (FDG-PET) in converters relative to those in nonconverters. 

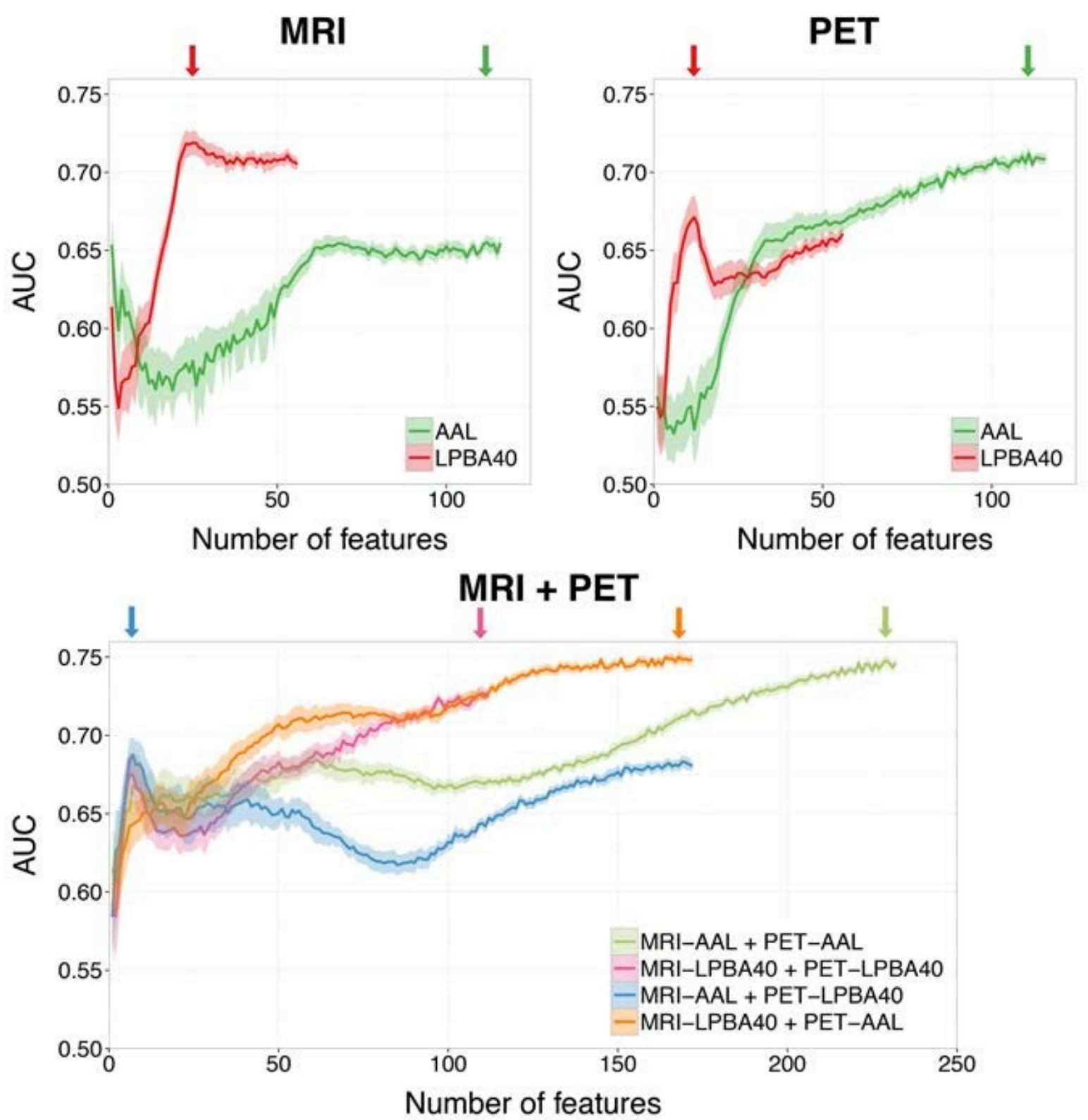

Fig. 3 Classification of performance of patients with mild cognitive impairment (MCI) by using support vector machine feature elimination (SVM-RFE) feature selection. Plotted are the mean areas under the receiver operating characteristic (ROC) curves (AUC) resulting from leave-one-out cross-validation tests for MCI classification using magnetic resonance imaging (MRI)-based, positron emission tomography (PET)-based, and multimodal feature sets with respect to the number of features. The shaded region of each plot indicates $95 \%$ confidence intervals. The arrows on the top of each plot represent the number of features that provided the highest AUC for each plot in the same color. 

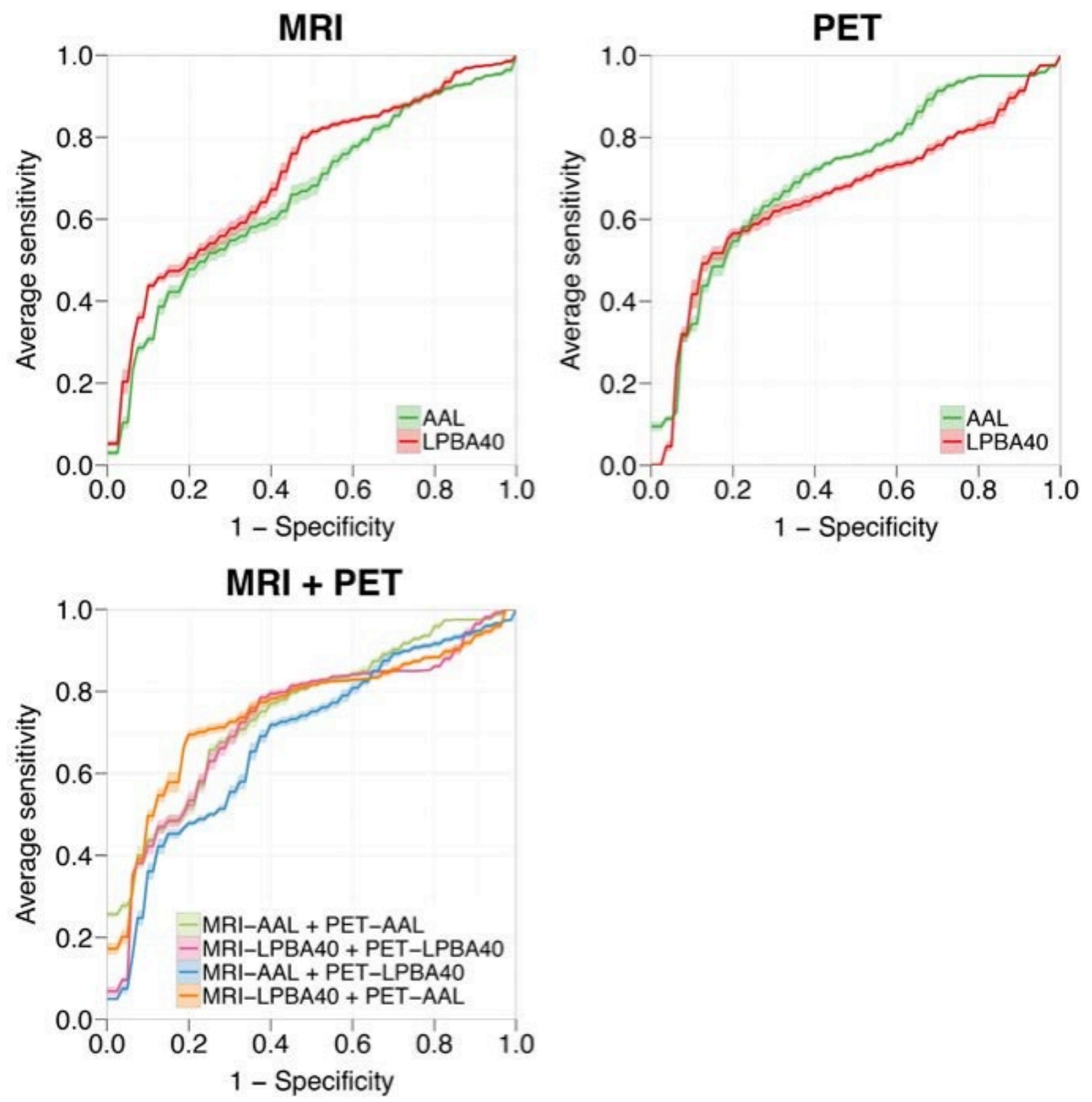

Fig. 4 Receiver operating characteristic (ROC) curves of eight original feature sets with no feature selection for classification of patients with mild cognitive impairment (MCI). Each curve was formed by vertically averaging 20 ROC curves that resulted from leave-one-out cross-validation tests. The shaded region of each curve indicates $95 \%$ confidence intervals. 


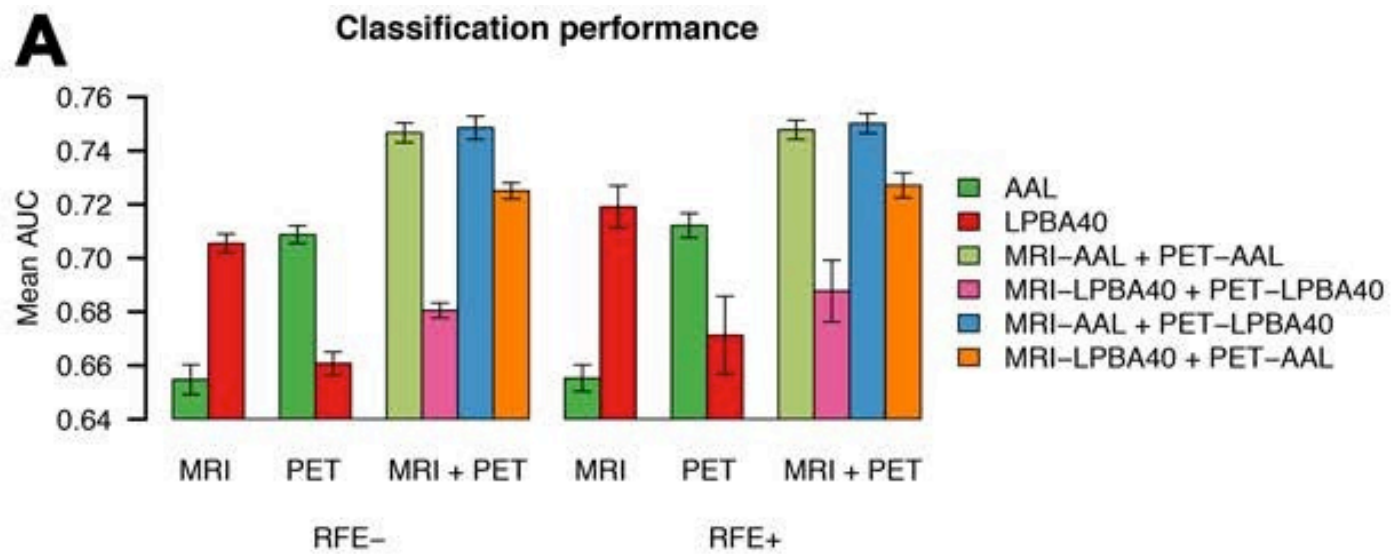

B

Three-way ANOVA Comparisons for modality
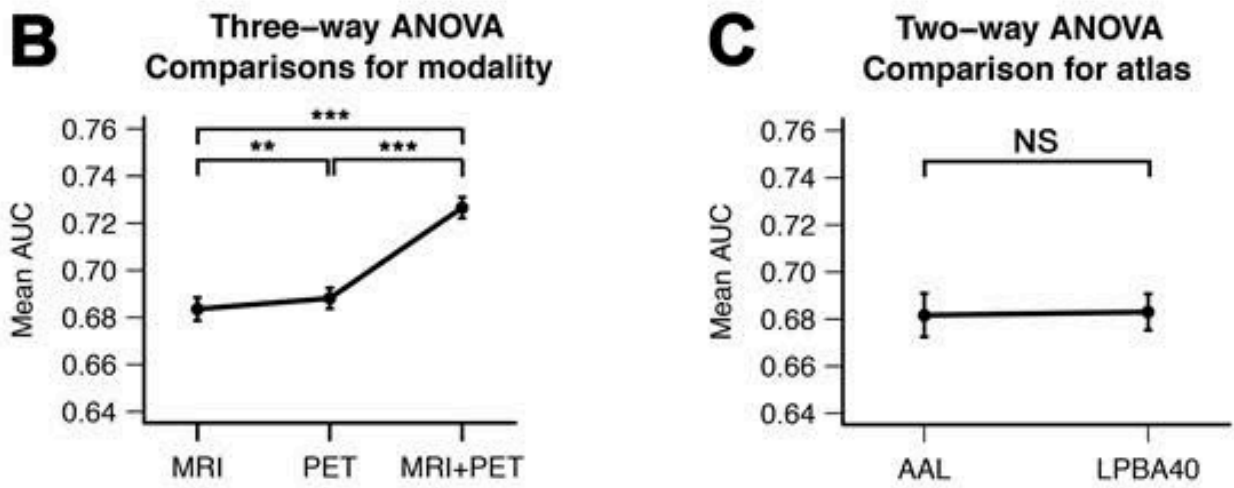

D

Modality

Unimodal feature sets

Atlas Interaction
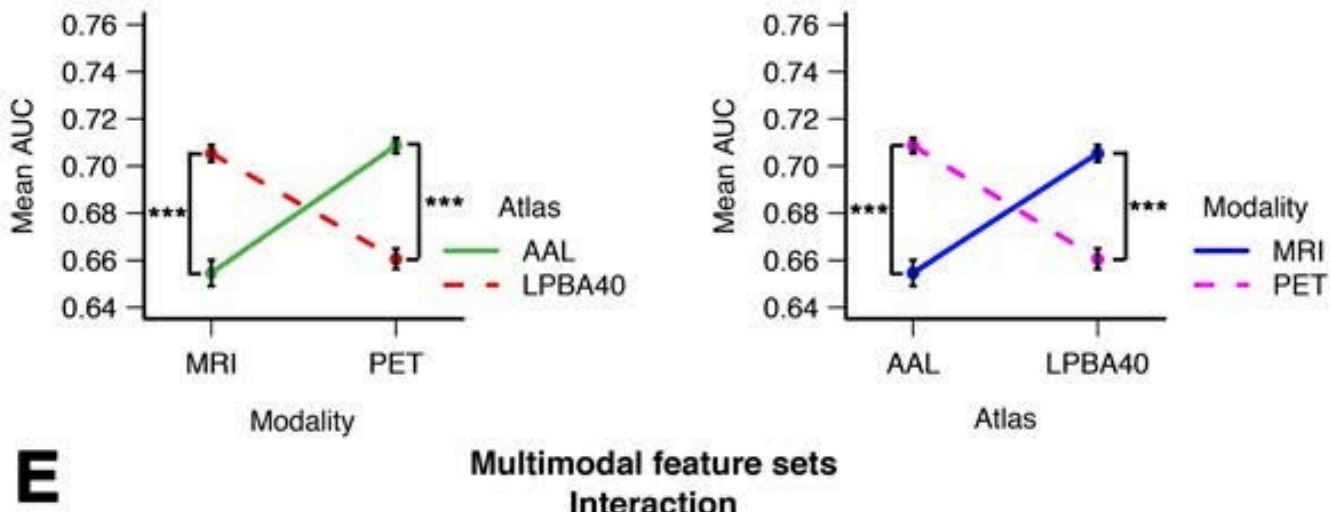

Multimodal feature sets

Interaction

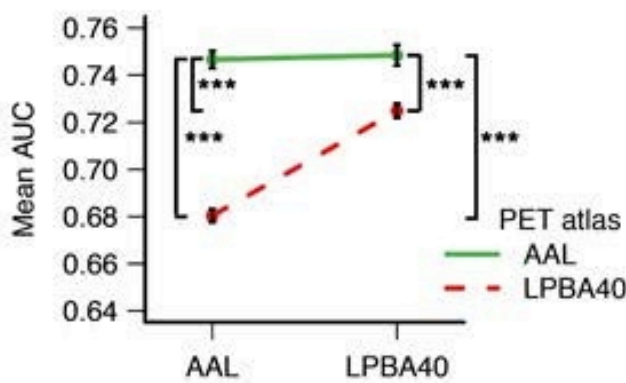

MRI atlas

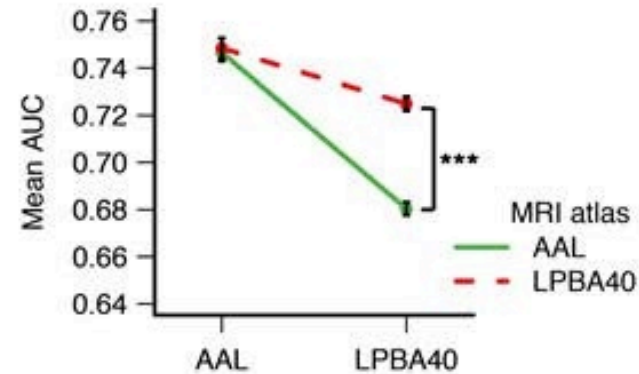

PET atlas

Fig. 5 Statistical analyses. The error bars are 95\% confidence intervals. (A) Classification performance. Mean $\mathrm{AUC}_{\mathrm{RFE}}$ and $\mathrm{AUC}_{\mathrm{RFE}}$ values. (B) Comparisons for modality in our 
three-way ANOVA. Post-hoc multiple comparisons revealed significant differences in the means of RFE - and RFE + between MRI and PET $(p=0.009)$, between MRI and MRI + PET $\left(p<10^{-7}\right)$, and between PET and MRI + PET $\left(p<10^{-7}\right)$. (C) Comparison of atlas type in our two-way (atlas $\times$ modality) ANOVA for unimodal feature sets. The main effect of atlas was not significant $\left[F(1,76)=0.43, p=0.51, \eta^{2}=0.0006\right]$. (D) Interaction plots of the two-way ANOVA for unimodal feature sets. Mean $\mathrm{AUC}_{\mathrm{RFE}}-$ values for different modalities and for different atlases in unimodal feature sets tested by the additional two-way (atlas $\times$ modality) ANOVA. The interaction between atlas and modality was significant $[F(1,76)=593.4, p<$ $\left.10^{-15}, \eta^{2}=0.88\right]$. All of the simple main effects of modality for $\operatorname{AAL}[F(1,76)=355.1, p<$ $\left.10^{-15}, \eta^{2}=0.53\right]$ and for LPBA40 $\left[F(1,76)=243.5, p<10^{-15}, \eta^{2}=0.36\right]$ and of atlas for MRI $\left[F(1,76)=312.9, p<10^{-15}, \eta^{2}=0.46\right]$ and for PET $\left[F(1,76)=280.9, p<10^{-15}, \eta^{2}=\right.$ 0.42] were significant. (E) Interaction plots of the two-way ANOVA for multimodal feature sets. The mean $\mathrm{AUC}_{\mathrm{RFE}}$ - values for different atlases for MRI or PET in multimodal feature sets were tested by another two-way (atlas for MRI $\times$ atlas for PET) ANOVA. The interaction between atlases for MRI and PET was significant $\left[F(1,76)=162.3, p<10^{-15}, \eta^{2}\right.$ $=0.14]$. The simple main effect of atlas for MRI at AAL for PET was not significant $[F$ $\left.(1,76)=0.60, p=0.44, \eta^{2}=0.0005\right]$, whereas we found the significant simple main effects of atlas for MRI at LPBA40 for PET $\left[F(1,76)=353.3, p<10^{-15}, \eta^{2}=0.31\right]$, of atlas for PET at AAL for MRI $\left[F(1,76)=780.1, p<10^{-15}, \eta^{2}=0.68\right]$, and of atlas for PET at LPBA40 for MRI $\left[F(1,76)=98.2, p<10^{-14}, \eta^{2}=0.09\right] . * * p<0.01, * * * p<0.001$. AUC, area under the receiver operating characteristic (ROC) curve; MRI, magnetic resonance imaging; PET, positron emission tomography; ANOVA, analysis of variance; AAL, Automated Anatomical Labeling; LPBA40, LONI Probabilistic Brain Atlas; RFE, recursive feature elimination; NS, not significant. 

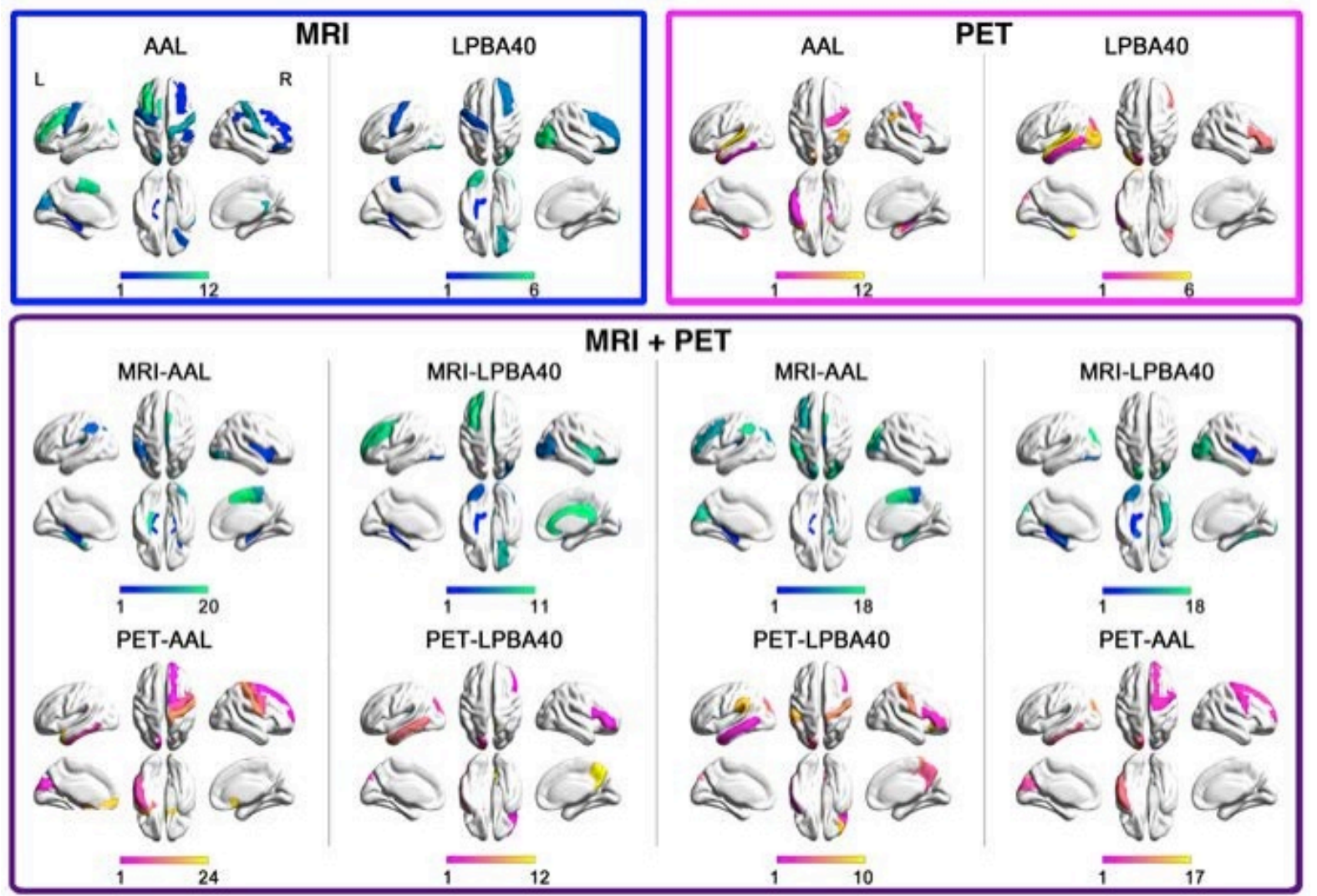

Fig. 6 Top 10\% ranked cortical regions for eight feature sets according to support vector machine-based recursive feature elimination feature selection. The cerebellar regions are not shown for simplicity. MRI, magnetic resonance imaging; PET, positron emission tomography; AAL, Automated Anatomical Labeling; LPBA40, LONI Probabilistic Brain Atlas. 


\section{Supplementary Material}

\section{Supplementary Materials and methods}

Prediction of conversion using the SEAD-J subjects' cognitive scores

We evaluated the prediction accuracy using the neuropsychological test scores (MMSE, WMS-R LM I, II, ADAS-Jcog, GDS, and a combined set of these scores) of SEAD$\mathrm{J}$ subjects in the same manner as imaging biomarkers. In addition, We used combined feature sets of cognitive scores and imaging biomarkers to examine whether combing cognitive scores and imaging biomarkers enhances prediction performance.

Evaluation using ADNI dataset: Participants

To evaluate our atlas-based method on a different dataset, we applied the same method to the ADNI dataset. We identified 158 individuals with amnestic mild cognitive impairment (aMCI) from participants in the Alzheimer's Disease Neuroimaging Initiative (ADNI). All the subjects (Supplementary Table 6) underwent screening (baseline) T1weighted MRI scans and FDG-PET scans. "Conversion” was defined as a change in diagnosis from aMCI to AD during a 36-month follow-up period, and 77 participants (\%) converted to AD within 36 months. Supplementary Table 7 shows more details on the ADNI participants' characteristics at baseline. The converter group (MCI-C) and nonconverter group (MCI-NC) significantly differed in baseline neuropsychological scores, including the Mini-Mental State Examination ( $p=0.0096, r=0.21$ ) and Alzheimer's Disease Assessment Scale-Cognitive Subscale ( $p<0.001, r=0.47$ ). No significant differences were observed in 
age $(p=0.85, \mathrm{r}=0.02)$, gender $\left(\chi^{2}\right.$ test, $\left.p=0.16, \mathrm{w}=0.11\right)$, and education $(p=0.57, \mathrm{r}=$ 0.05). Student's $t$-test was used to compare baseline differences between the two groups unless otherwise stated. We computed $\mathrm{r}$ for the t-test and $\mathrm{w}$ for the $\chi^{2}$ test as measures of effect size.

Magnetic resonance image acquisition and preprocessing

Three-dimensional structural MRI scans were downloaded from the Laboratory of Neuro Imaging (LONI) Image Data Archive (https://ida.loni.usc.edu/). These structural MRI scans were acquired from 1.5-T MRI scanners. We performed the same voxel-based morphometry (VBM) preprocessing procedures using the SPM8 software package (http://www.fil.ion.ucl.ac.uk/spm/software/spm8) and VBM8 Toolbox (http://dbm.neuro.unijena.de/vbm) in MATLAB 7.12 as described previously (Ota et el., 2014).

Fluorodeoxyglucose positron emission tomography image acquisition and preprocessing

Fluorodeoxyglucose positron emission tomography (FDG-PET) scans were downloaded from the LONI Image Data Archive. The whole brain was used as the reference region for intensity normalization. The images were normalized by using an in-house FDGPET template and smoothed in the same manner as the SEAD-J images.

\section{Feature extraction}

Using the Automated Anatomical Labeling (AAL) atlas (Tzourio-Mazoyer et al., 2002) and the LONI Probabilistic Brain Atlas (LPBA40) (Shattuck et al., 2008), we extracted 
feature vectors from the preprocessed baseline MRI and from the FDG-PET data in the same manner as the SEAD-J data to obtain eight different feature sets, including two MR-based features (MRI-AAL and MRI-LPBA40) representing GM density in each ROI, two PETbased features (PET-AAL and PET-LPBA40) representing relative cerebral metabolic rate for glucose in each ROI, and four multimodal features (MRI-AAL + PET-AAL, MRI-AAL + PET-LPBA40, MRI-LPBA40 + PET-AAL, and MRI-LPBA40 + PET-LPBA40).

\section{Classification}

We used linear SVMs with the regularization parameter $\mathrm{C}$ of 1 in the same manner as in the SEAD-J analysis. To enable statistical analysis of the results, we used a bootstrap aggregating (bagging) method (Breiman, 1996) within each round of LOOCV (Dosenbach et al., 2010). In each LOOCV loop, the original data set having 158 participants was divided into a test set of one participant and a training set comprising 157 participants. Then a bootstrap sample of $90 \%$ of the total number of participants was obtained by bootstrap resampling of the original training set. For the eight different feature sets, we repeated the above LOOCV procedure 20 times and computed mean AUC values for AD prediction across 20 LOOCV tests in the same manner as in the SEAD-J analysis. To compare the ability of prediction across different feature sets, we formed averaged ROC curves with 95\% confidence intervals for each feature set on the basis of the results of 20 LOOCV tests by using Fawcett’s vertical averaging algorithm (Fawcett, 2004).

\section{Feature selection}


We applied the multiple SVM-RFE (MSVM-RFE) feature selection method (Duan et al., 2005) to compute the feature ranking score for a given feature from weight vectors of multiple linear SVMs trained on bootstrap subsamples of the original training data in the same manner as in the SEAD-J analysis.

\section{Statistical analysis}

To test the differences between the means of ADNI AUC values for eight feature sets, we used $A U C_{R F E-}$ and $A U C_{R F E+}$ for each feature set in the same manner as in the SEAD-J study. $A U C_{R F E-}$ for a feature set was an AUC value that was obtained with the original features (with no feature selection), and $\mathrm{AUC}_{\mathrm{RFE}}+$ was determined as the highest AUC during the SVM-RFE procedure for the feature set. We performed an overall three-way factorial analysis of variance (ANOVA) that included all the factors in the same manner as in the SEAD-J study. The R Statistical Computing Environment, version 3.0.2 (R Development Core Team, 2013) was used for classification, feature selection, and all statistical analyses.

\section{Supplementary Results}

Prediction using the SEAD-J subjects' cognitive scores

Supplementary Fig. 1 shows the ROC curves for MCI classification for cognitive feature sets of the SEAD-J subjects (bottom right). WMS-R LM II provided the highest AUC value (0.767) among the cognitive feature sets, followed by the combination of all the test scores (0.761), WMS-R LM I (0.729), MMSE (0.699), GDS (0.652), and ADAS-Jcog (0.650). 
We conducted three-way (modality $\times$ atlas $\times$ cognitive test) ANOVA, resulting in the significant three-way interaction $\left[F(30,1368)=36.4, p<0.001, \eta^{2}=0.041\right]$. Our post hoc multiple comparisons revealed significant differences between any of the different groups ( $p$ $<0.001$ ) except between WMS-R LM II and All test scores and between GDS and ADASJcog.

Some of the combined feature sets of cognitive scores and imaging biomarkers outperformed cognitive scores alone or imaging biomarkers alone, and the performance varied depending on how to combine these measures (Supplementary Figs. 2 and 3).

Effects of imaging modality, brain atlas, and support vector machine recursive feature elimination on prediction using the ADNI dataset

As shown in Supplementary Figs. 4 to 6, the results of ADNI were different from those of SEAD-J. For MRI-based features, AAL was found to be better than LPBA40, and vice versa for PET-based features. No apparent effect of combining multimodal features in improving prediction performance was shown in the ADNI dataset.

Our three-way ANOVA demonstrated a significant three-way interaction $[F(6,479)$ $=42.5, p<0.001, \eta^{2}=0.049$ ] (Supplementary Table 8). Two-way interactions at various levels of each factor were also all significant (Supplementary Tables 9 to 11). Simple main effects were all significant except for atlas at PET at RFE+ (Supplementary Tables 12 to 14).

Important regions determined by support vector machine recursive feature elimination feature selection using the ADNI dataset 
Supplementary Tables 15 and 16 lists the regions determined by the SVM-RFE feature selection method using the SEAD-J and ADNI datasets, respectively. The label abbreviations are listed in Table 6. Top selected regions were different from these datasets. 


\section{Supplementary References}

Breiman L. Bagging Predictors. Mach Learn 1996;24:123-140.

Dosenbach NUF, Nardos B, Cohen AL, Fair DA, Power JD, Church JA, et al. Prediction of individual brain maturity using fMRI. Science 2010;329:1358-1361.

Duan K-B, Rajapakse JC, Wang H, Azuaje F. Multiple SVM-RFE for gene selection in cancer classification with expression data. IEEE Trans.on Nanobioscience 2005;4:228-234.

Fawcett T. ROC Graphs: Notes and Practical Considerations for Researchers. Mach Learn 2004:1-38.

Ota K, Oishi N, Ito K, Fukuyama H, SEAD-J Study Group. A comparison of three brain atlases for MCI prediction. J Neurosci Methods 2014;221:139-150.

R Development Core Team. R: A Language and Environment for Statistical Computing. Vienna: R Foundation for Statistical Computing; 2013.

Shattuck DW, Mirza M, Adisetiyo V, Hojatkashani C, Salamon G, Narr KL, et al. Construction of a 3D probabilistic atlas of human cortical structures. Neuroimage 2008;39:1064-1080.

Tzourio-Mazoyer N, Landeau B, Papathanassiou D, Crivello F, Etard O, Delcroix N, et al. Automated Anatomical Labeling of Activations in SPM Using a Macroscopic Anatomical Parcellation of the MNI MRI Single-Subject Brain. Neuroimage 2002;15:273-289. 


\section{Supplementary Figure Captions}

Supplementary Fig. 1 Receiver operating characteristic (ROC) curves for cognitive scores and eight original feature sets with no feature selection for classification of the Studies on Diagnosis of Early Alzheimer’s Disease-Japan (SEAD-J) subjects with mild cognitive impairment (MCI) (Fig. 4). Each curve was formed by vertically averaging 20 ROC curves that resulted from leave-one-out cross-validation tests. The shaded region of each curve indicates 95\% confidence intervals.

Supplementary Fig. 2 Receiver operating characteristic (ROC) curves for combined feature sets of unimodal imaging biomarkers and cognitive scores with no feature selection for classification of the Studies on Diagnosis of Early Alzheimer's Disease-Japan (SEAD-J) subjects with mild cognitive impairment (MCI). Each curve was formed by vertically averaging 20 ROC curves that resulted from leave-one-out cross-validation tests. The shaded region of each curve indicates 95\% confidence intervals.

Supplementary Fig. 3 Receiver operating characteristic (ROC) curves for combined feature sets of multimodal imaging biomarkers and cognitive scores with no feature selection for classification of the Studies on Diagnosis of Early Alzheimer's Disease-Japan (SEAD-J) subjects with mild cognitive impairment (MCI). Each curve was formed by vertically averaging 20 ROC curves that resulted from leave-one-out cross-validation tests. The shaded region of each curve indicates $95 \%$ confidence intervals.

Supplementary Fig. 4 Comparison of classification performance of patients with mild cognitive impairment (MCI) by using support vector machine feature elimination (SVM- 
RFE) feature selection between the Studies on Diagnosis of Early Alzheimer's Disease-Japan (SEAD-J) and the Alzheimer's Disease Neuroimaging Initiative (ADNI) datasets. Plotted are the mean areas under the receiver operating characteristic (ROC) curves (AUC) resulting from leave-one-out cross-validation tests for MCI classification using magnetic resonance imaging (MRI)-based, positron emission tomography (PET)-based, and multimodal feature sets with respect to the number of features. The shaded region of each plot indicates $95 \%$ confidence intervals.

Supplementary Fig. 5 Comparison between the Studies on Diagnosis of Early Alzheimer's Disease-Japan (SEAD-J) and the Alzheimer's Disease Neuroimaging Initiative (ADNI) datasets: Receiver operating characteristic (ROC) curves of eight original feature sets with no feature selection for classification of patients with mild cognitive impairment (MCI). Each curve was formed by vertically averaging 20 ROC curves that resulted from leave-one-out cross-validation tests. The shaded region of each curve indicates 95\% confidence intervals.

Supplementary Fig. 6 Comparison between the Studies on Diagnosis of Early Alzheimer’s Disease-Japan (SEAD-J) and the Alzheimer's Disease Neuroimaging Initiative (ADNI) datasets: Receiver operating characteristic (ROC) curves of eight original feature sets with support vector machine-based feature elimination (SVM-RFE) for classification of patients with mild cognitive impairment (MCI). Each curve was formed by vertically averaging 20 ROC curves that resulted from leave-one-out cross-validation tests. The shaded region of each curve indicates 95\% confidence intervals. 
S 2 京都大学ry Fig. 1
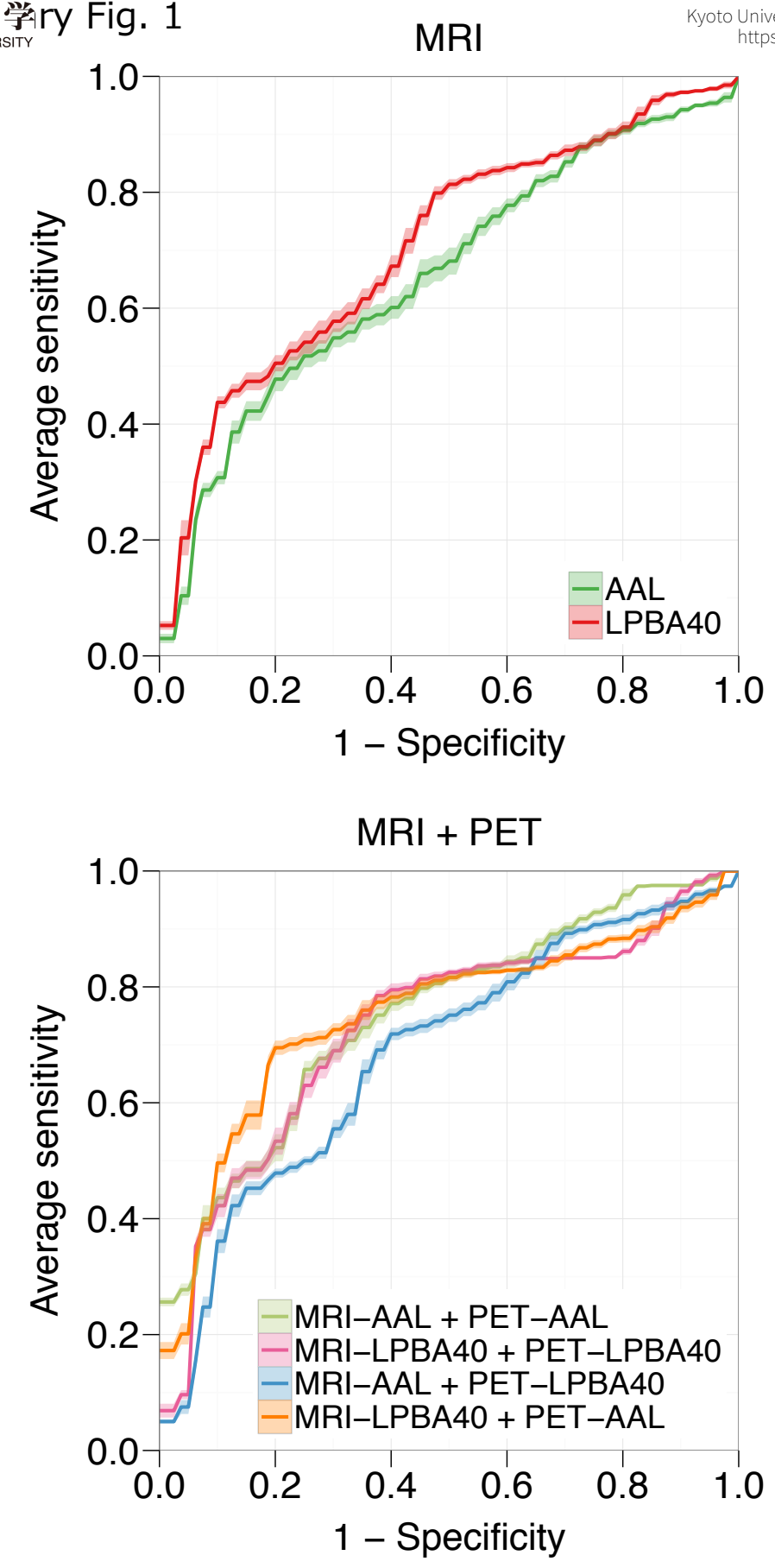

\section{PET}

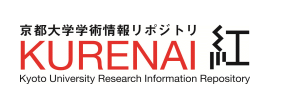
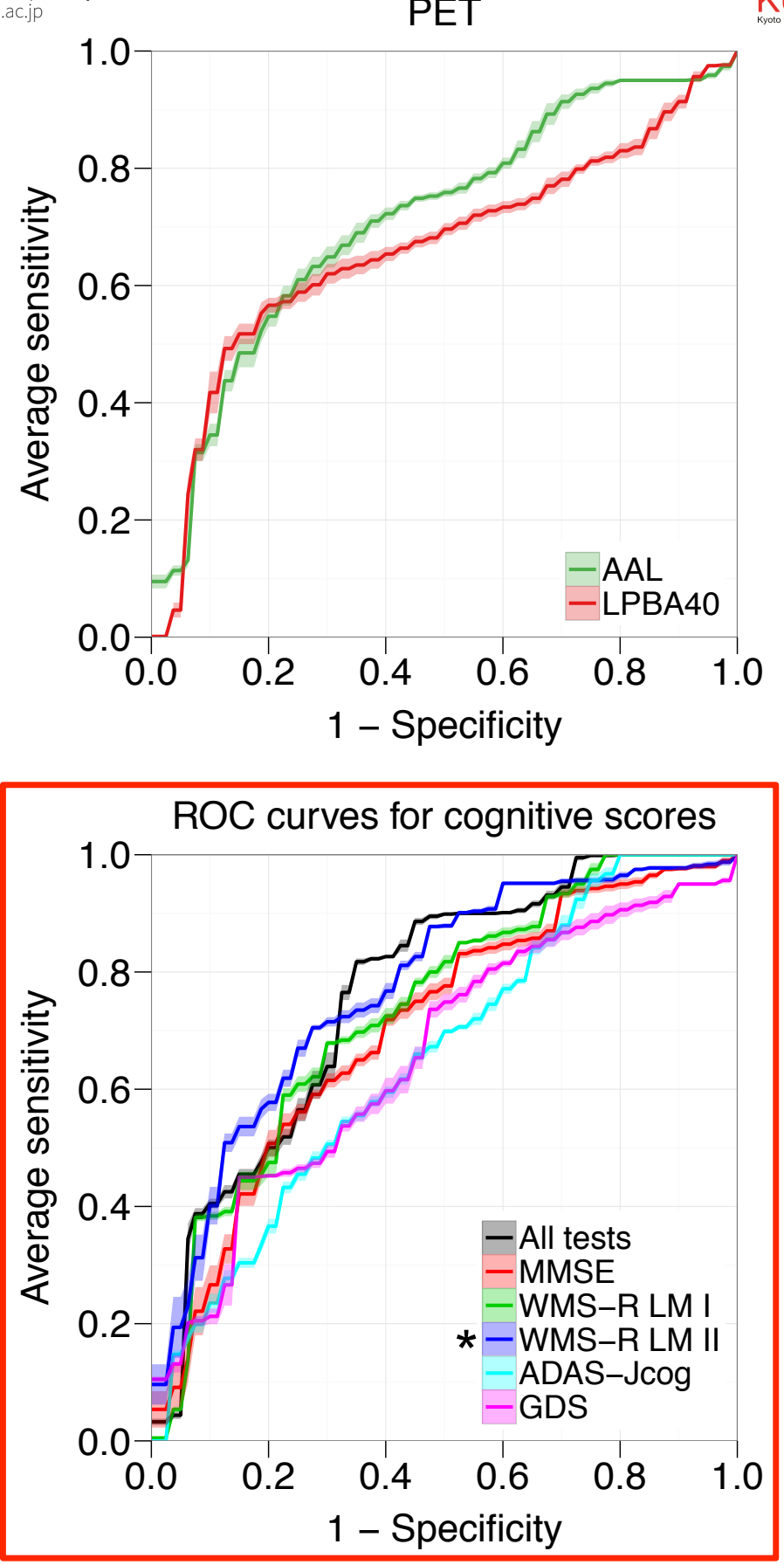
䅎ry Fig. 2
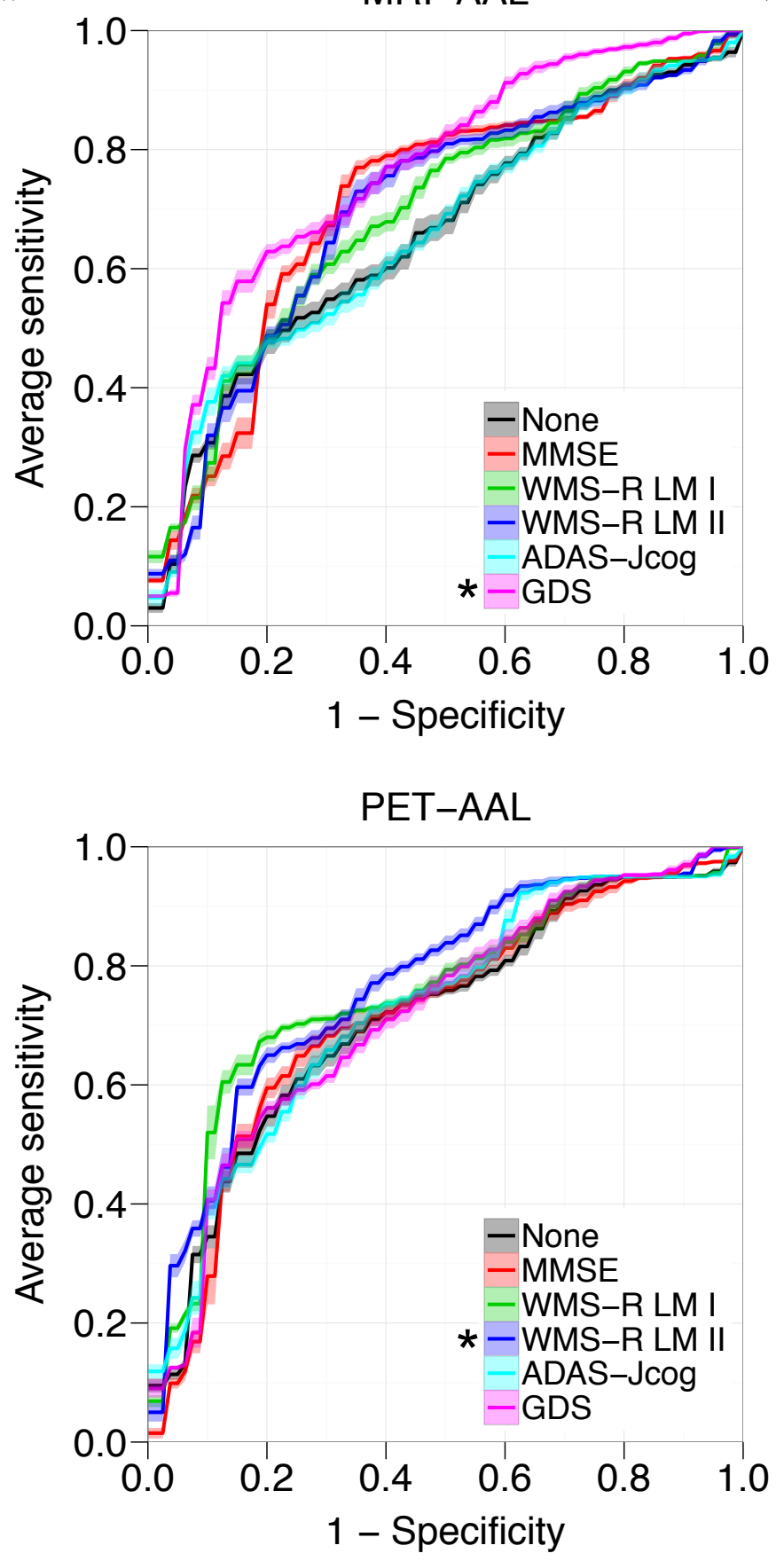

MRI-LPBA40

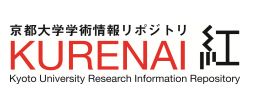
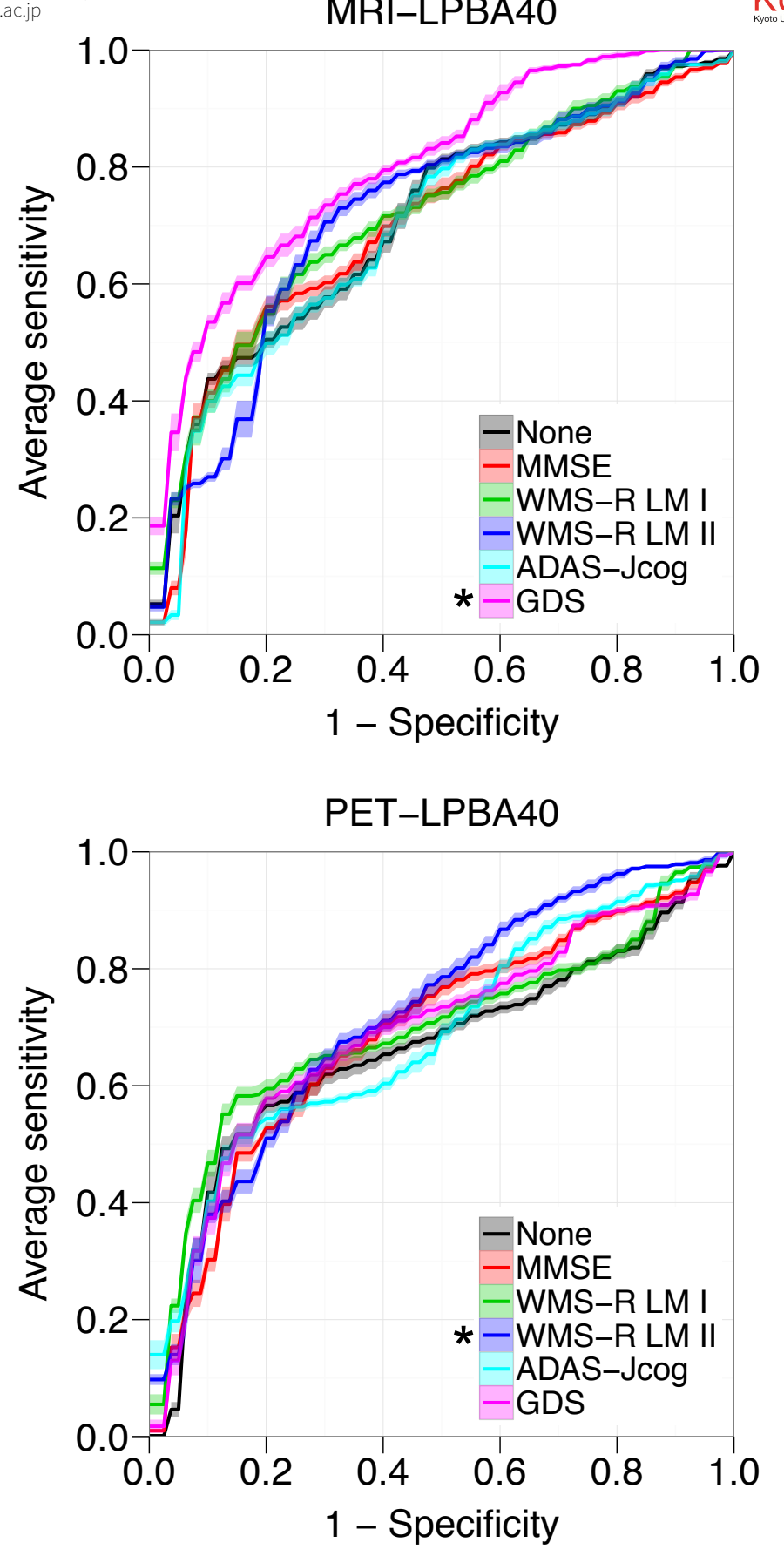

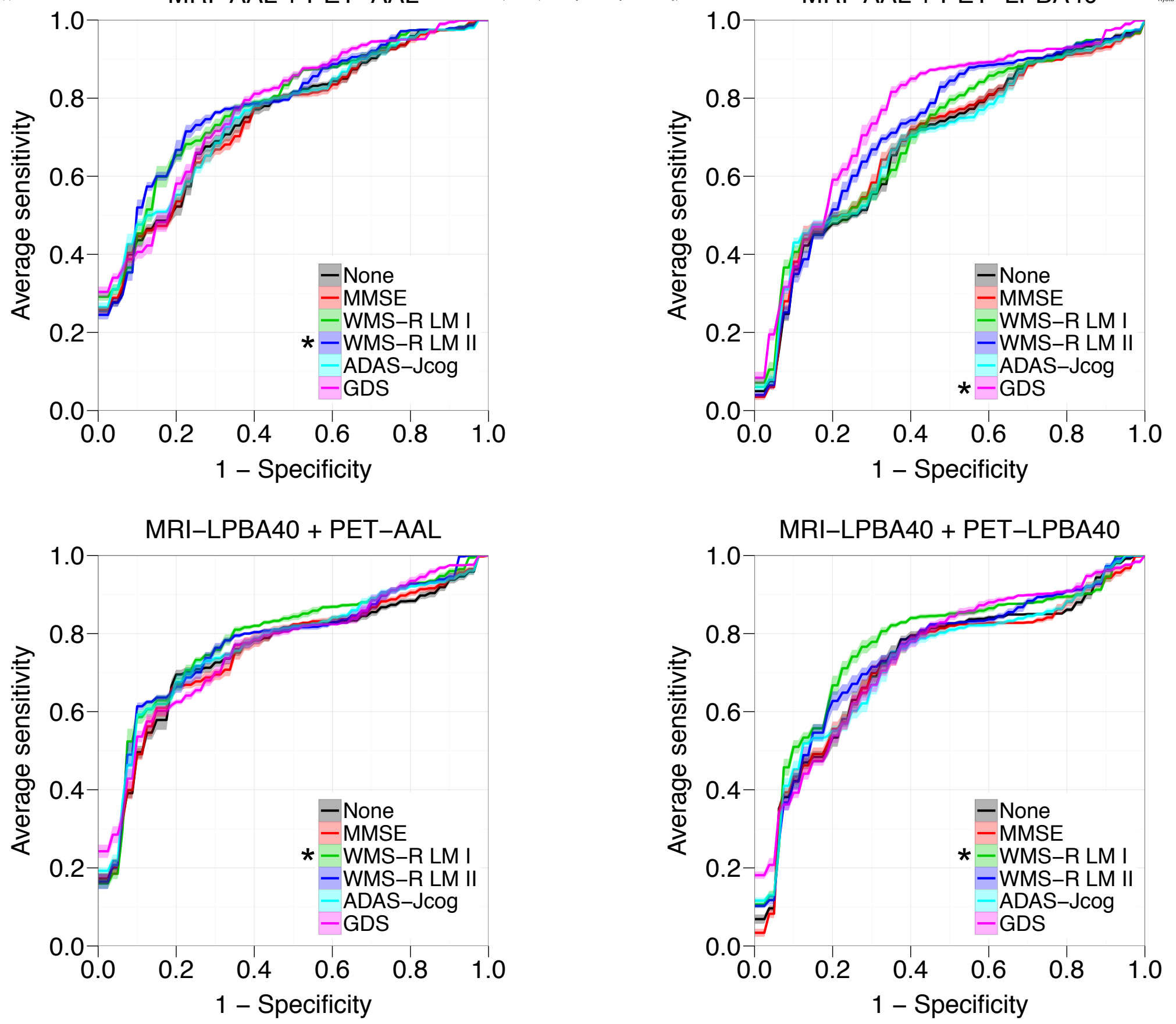


\section{Comparison between SEAD-J and ADNI - AUC vs. Number of features}

SEAD-J $(n=80)$
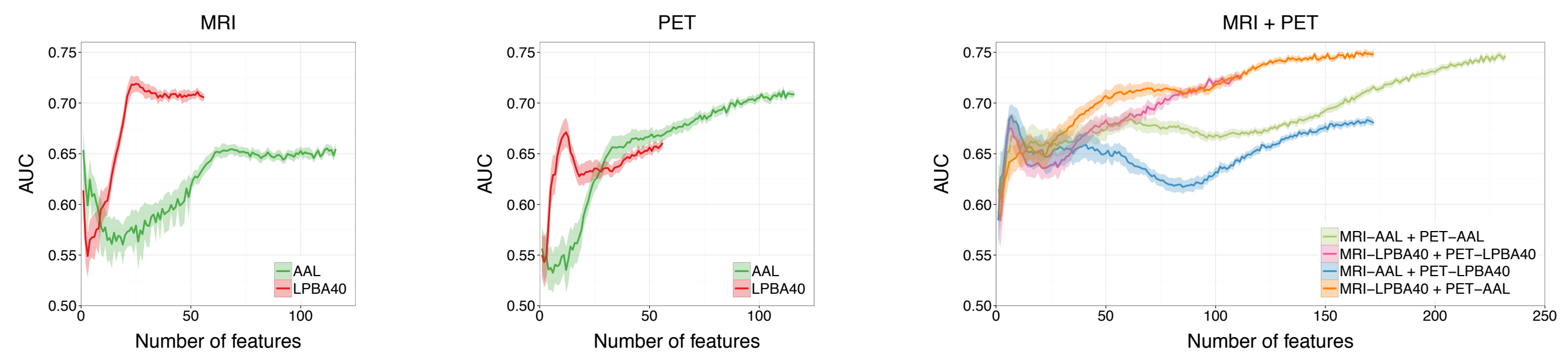

ADNI $(n=158)$
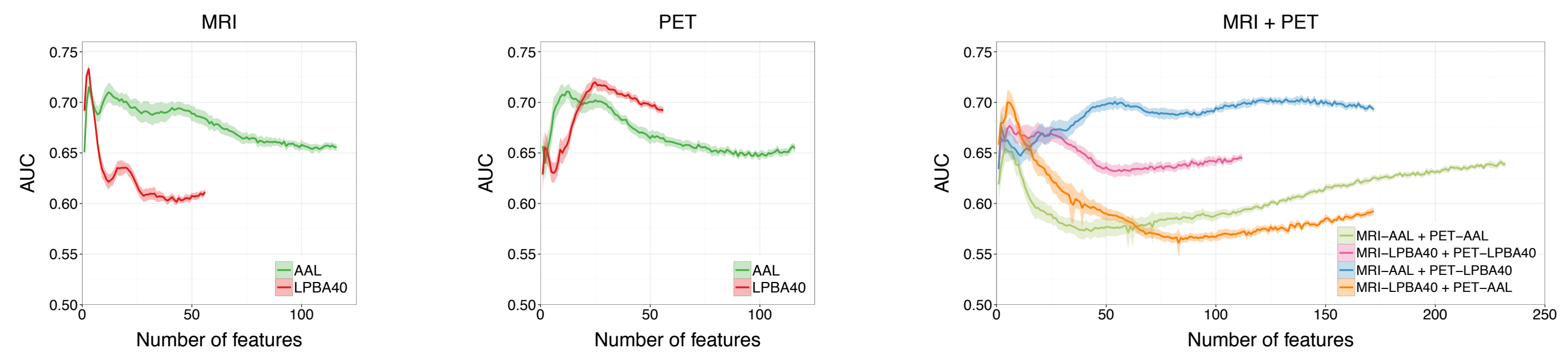


\section{Comparison between SEAD-J and ADNI - ROC curves (all features)}

SEAD-J $(\mathrm{n}=80)$

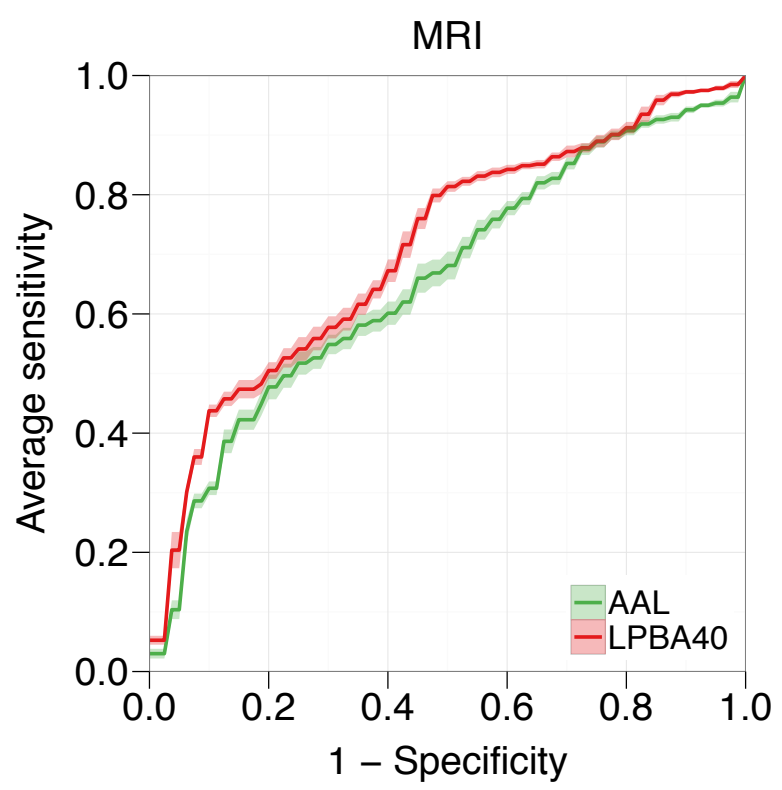

ADNI ( $n=158)$

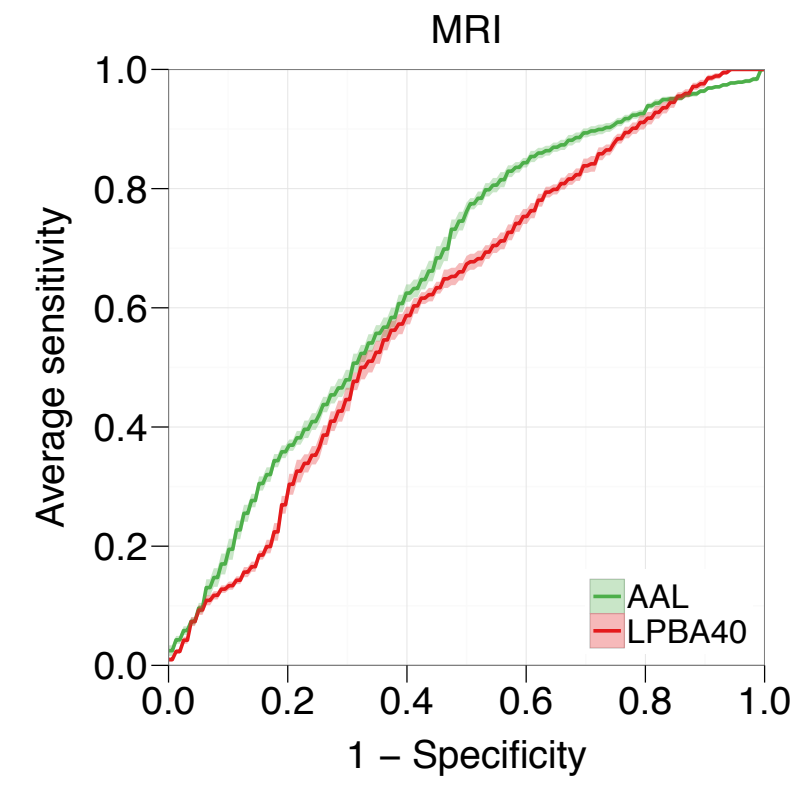

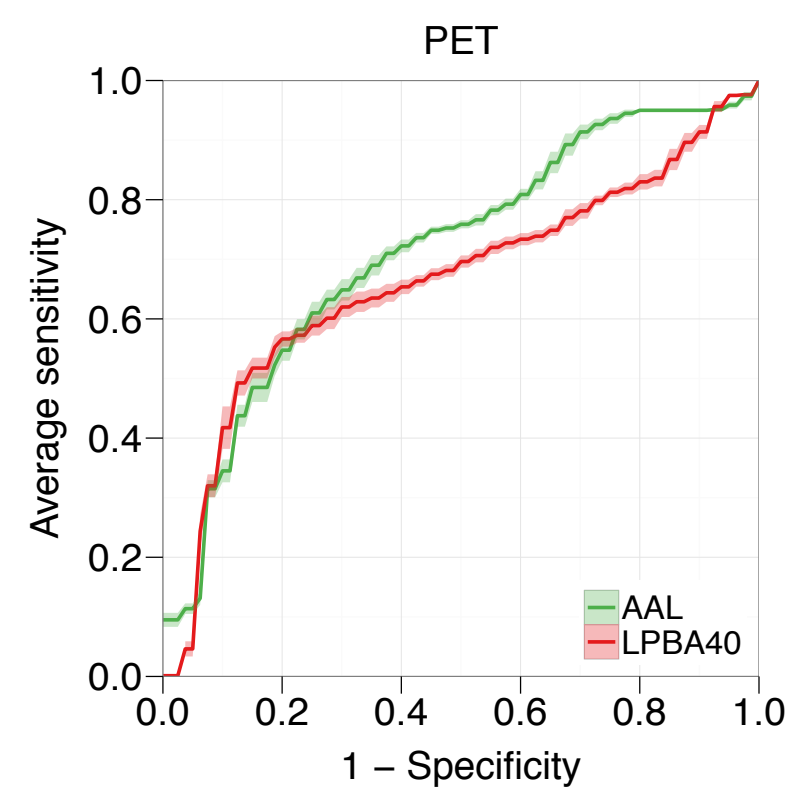

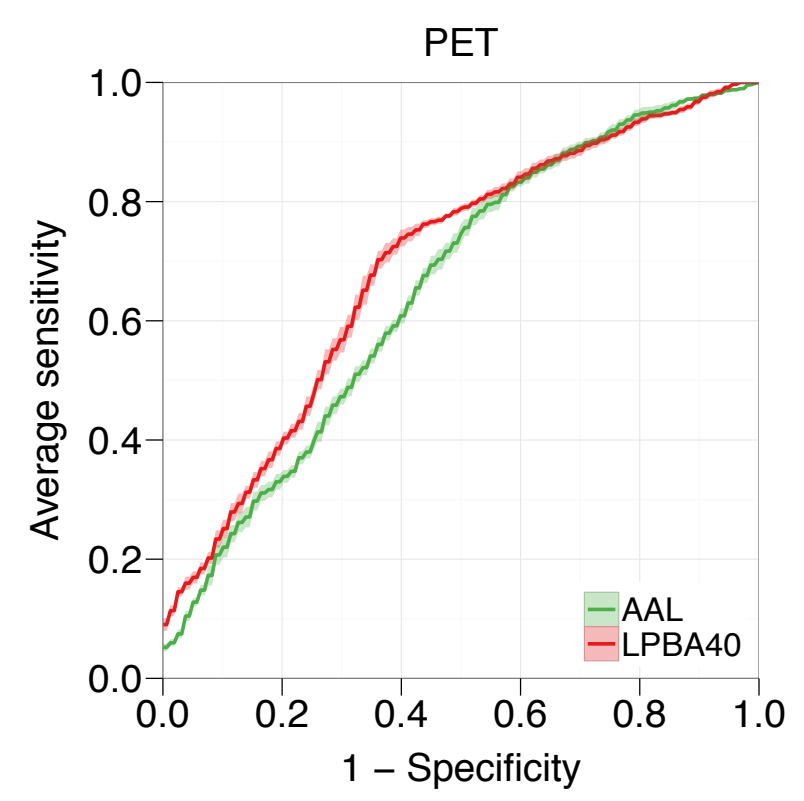

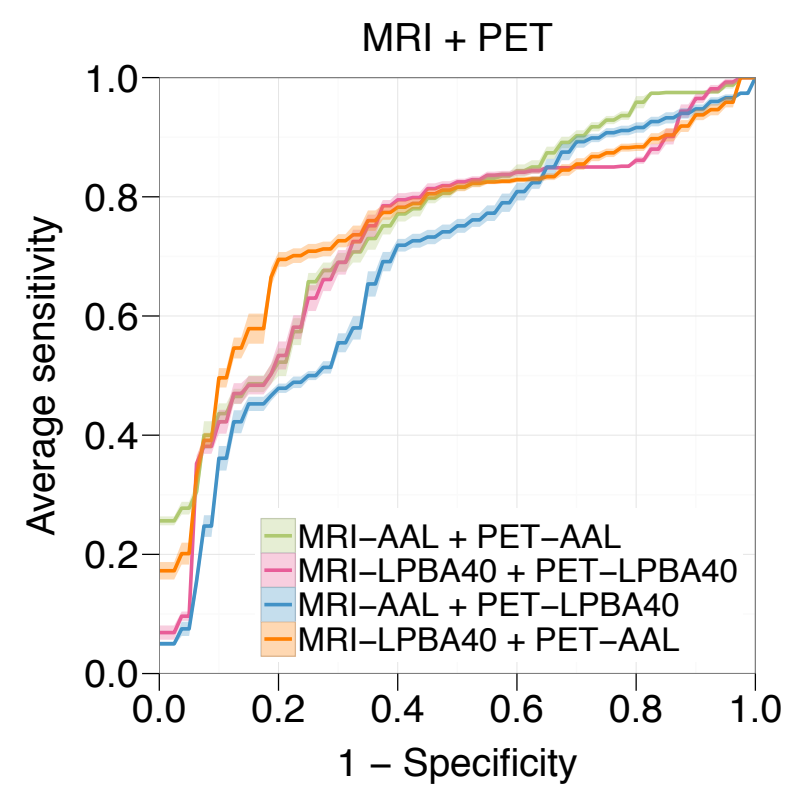

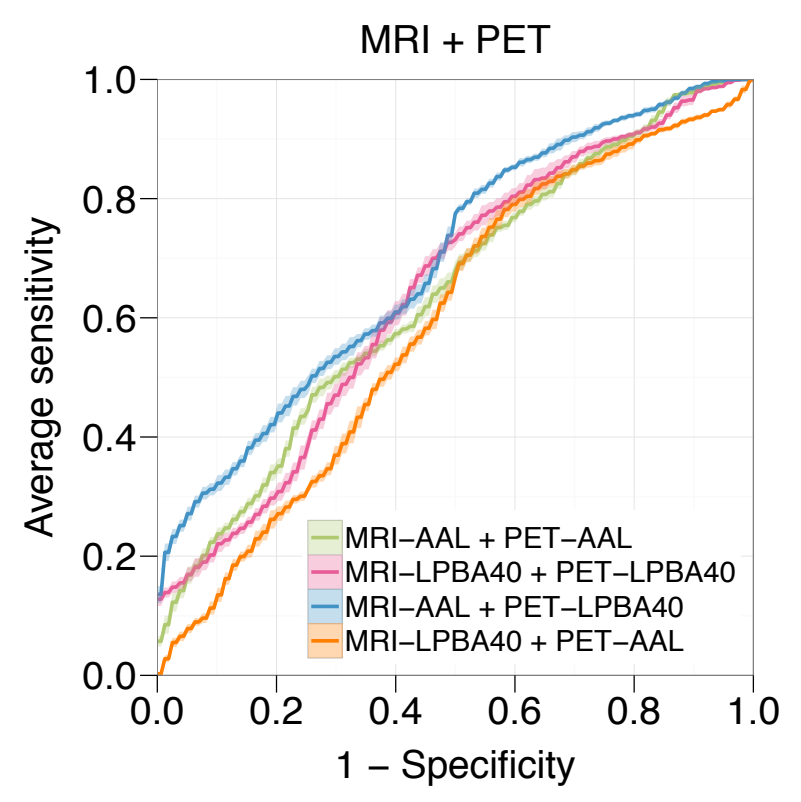




\section{Comparison between SEAD-J and ADNI - ROC curves (SVM-RFE)}

SEAD-J $(\mathrm{n}=80)$

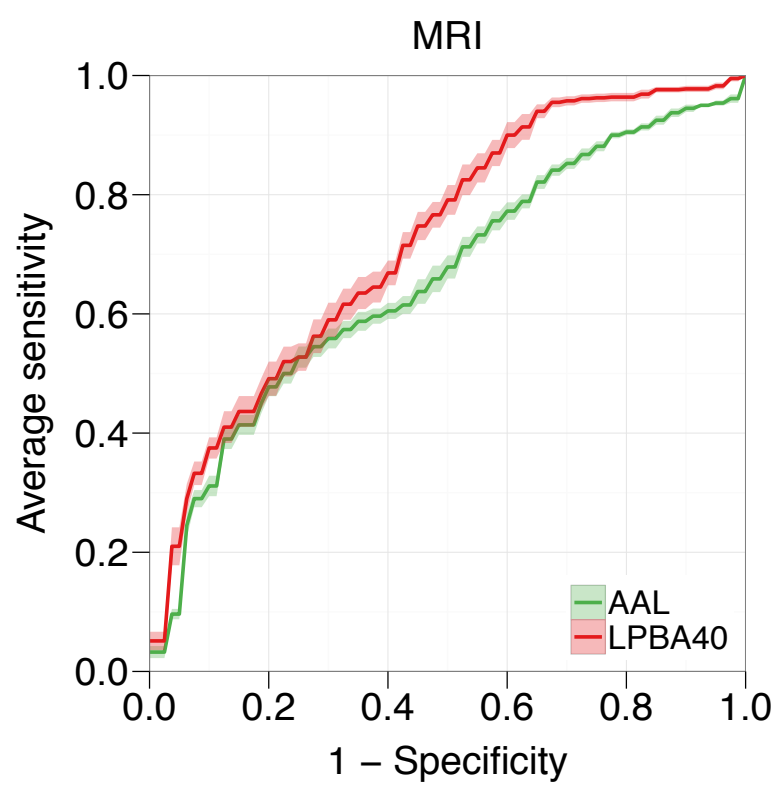

ADNI ( $n=158)$

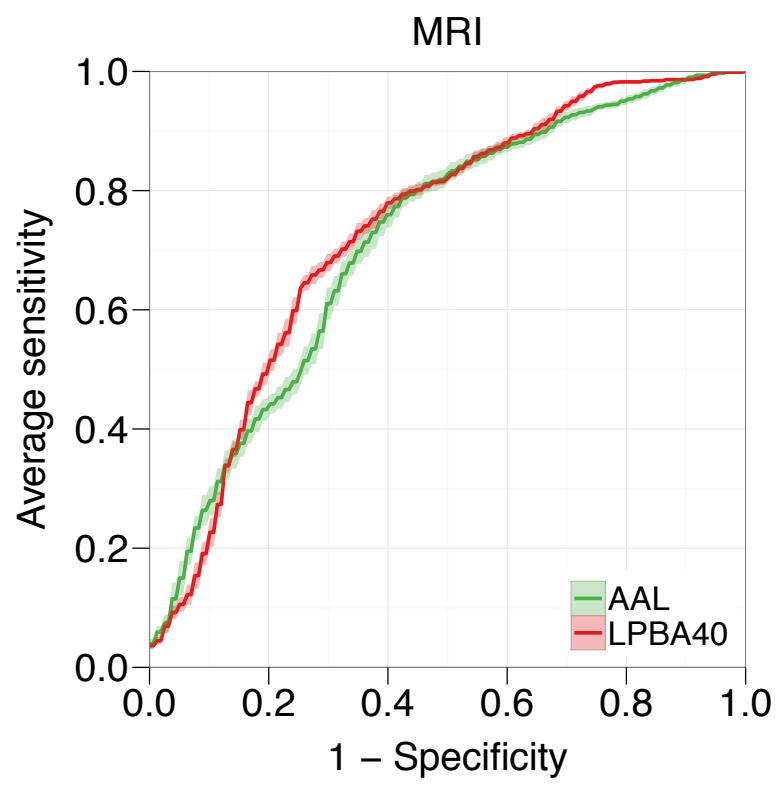

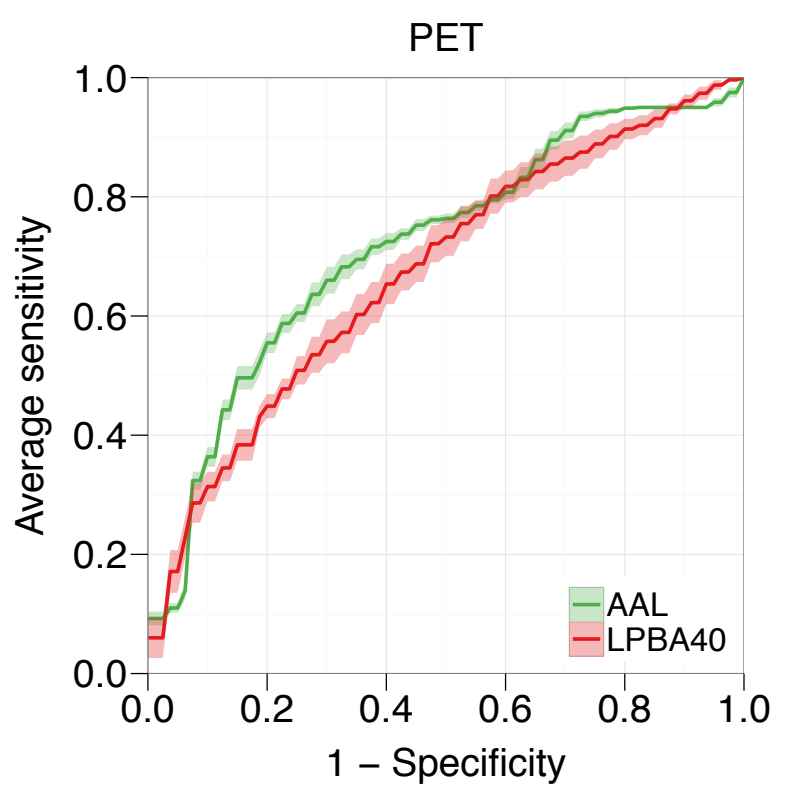

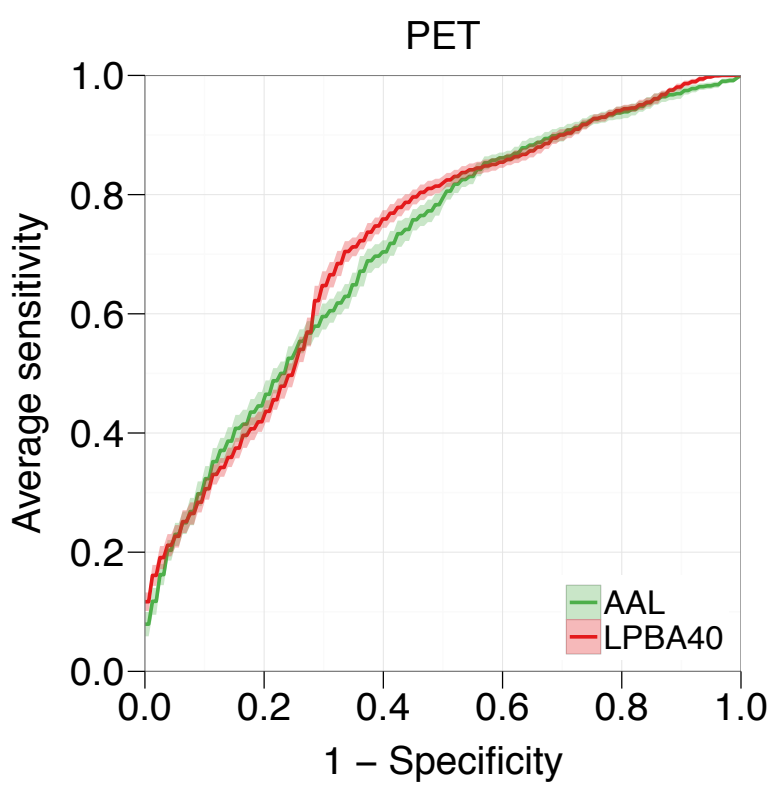

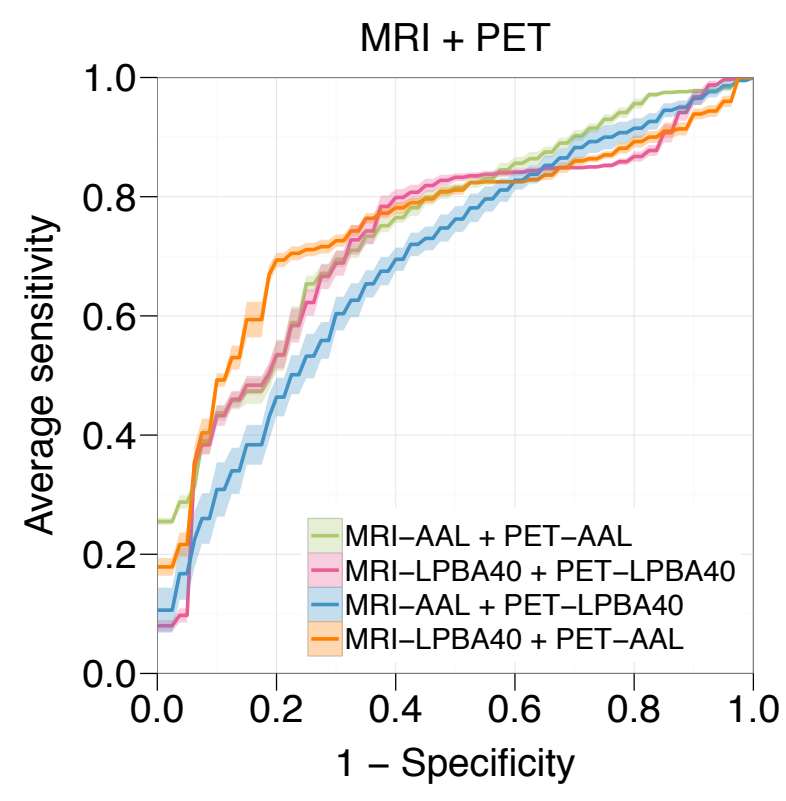

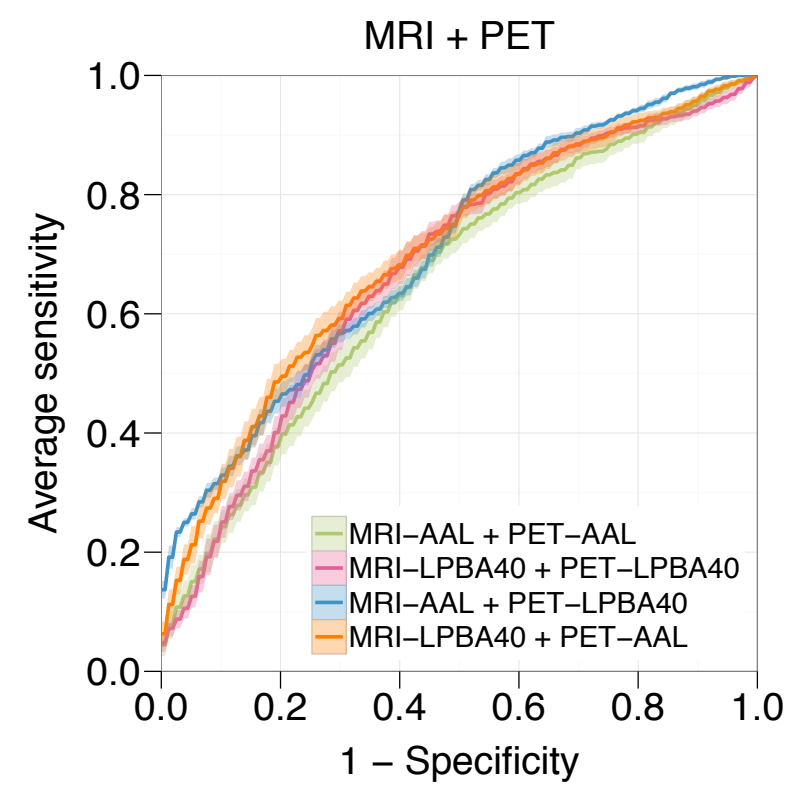




\section{Supplementary Table 1}

Researchers and institutions participating in the SEAD-J Study.

\begin{tabular}{|c|c|c|c|}
\hline & Researcher & Institution & Department \\
\hline 1 & Hidenao Fukuyama & Kyoto University Graduate School of Medicine & Human Brain Research Center \\
\hline 2 & Hirosi Yamauchi & Kyoto University Graduate School of Medicine & Human Brain Research Center \\
\hline 3 & Nobukatsu Sawamoto & Kyoto University Graduate School of Medicine & Human Brain Research Center \\
\hline 4 & Tosihiko Asou & Kyoto University Graduate School of Medicine & Human Brain Research Center \\
\hline 5 & Chihiro Namiki & Kyoto University Graduate School of Medicine & Human Brain Research Center \\
\hline 6 & Michio Senda & $\begin{array}{l}\text { Institute of Biomedical Research and } \\
\text { Innovation }\end{array}$ & Division of Molecular Imaging \\
\hline 7 & Takashi Kawachi & $\begin{array}{l}\text { Institute of Biomedical Research and } \\
\text { Innovation }\end{array}$ & Division of Molecular Imaging \\
\hline 8 & Tomohiko Yamane & $\begin{array}{l}\text { Institute of Biomedical Research and } \\
\text { Innovation }\end{array}$ & Division of Molecular Imaging \\
\hline 9 & Hidehito Nagai & $\begin{array}{l}\text { Institute of Biomedical Research and } \\
\text { Innovation }\end{array}$ & Division of Molecular Imaging \\
\hline 10 & Kiyoshi Maeda & Kobe Gakuin University & Department of Medical Rehabilitation \\
\hline 11 & Yasushi Yamamoto & Kobe University Graduate School of Medicine & Department of Psychiatry \\
\hline 12 & Yoshihiro Tahara & Kobe University Graduate School of Medicine & Department of Psychiatry \\
\hline 13 & Yasuomi Ouchi & $\begin{array}{l}\text { Medical Photonics Research Center, } \\
\text { Hamamatsu University School of Medicine }\end{array}$ & Department of Biofunctional Imaging \\
\hline 14 & Masanobu Sakamoto & Hamamatsu Medical Center & Department of neurology \\
\hline 15 & Toshihiko Kanno & Hamamatsu Medical Center & Positron Medical Center \\
\hline 16 & Kenichi Shimada & Hyogo Brain and Heart Center & $\begin{array}{l}\text { Institute for Aging Brain and Cognitive } \\
\text { Disorders }\end{array}$ \\
\hline 17 & Shingo Ohkawa & Hyogo Brain and Heart Center & $\begin{array}{l}\text { Institute for Aging Brain and Cognitive } \\
\text { Disorders }\end{array}$ \\
\hline 18 & Akira Terashima & Hyogo Brain and Heart Center & $\begin{array}{l}\text { Institute for Aging Brain and Cognitive } \\
\text { Disorders }\end{array}$ \\
\hline 19 & Yasuhito Higashi & Himeji central Hospital & Department of Neurology \\
\hline 20 & Kazunari Ishii & Kinki University School of Medicine & Department of Radiology \\
\hline 21 & Kenji Ishii & $\begin{array}{l}\text { Positron Medical Center, Tokyo Metropolitan } \\
\text { Institute of Gerontology }\end{array}$ & Positron Medical Center \\
\hline 22 & Masahiro Mishina & $\begin{array}{l}\text { Positron Medical Center, Tokyo Metropolitan } \\
\text { Institute of Gerontology }\end{array}$ & Positron Medical Center \\
\hline
\end{tabular}




\begin{tabular}{|c|c|c|c|}
\hline 23 & Masaya Hashimoto & $\begin{array}{l}\text { Positron Medical Center, Tokyo Metropolitan } \\
\text { Institute of Gerontology }\end{array}$ & Positron Medical Center \\
\hline 24 & Ayumu Okumura & $\begin{array}{l}\text { Chubu Medical Center for Prolonged } \\
\text { Traumatic Brain Dysfunction, Kizawa } \\
\text { Memorial Hospital }\end{array}$ & Department of Neurosurgery \\
\hline 25 & Yuka Okumura & $\begin{array}{l}\text { Chubu Medical Center for Prolonged } \\
\text { Traumatic Brain Dysfunction, Kizawa } \\
\text { Memorial Hospital }\end{array}$ & Department of Medical Technology \\
\hline 26 & Seisuke Fukuyama & $\begin{array}{l}\text { Chubu Medical Center for Prolonged } \\
\text { Traumatic Brain Dysfunction, Kizawa } \\
\text { Memorial Hospital }\end{array}$ & Department of Medical Technology \\
\hline 27 & Kengo Ito & $\begin{array}{l}\text { National Institute for Longevity Sciences, } \\
\text { National Center for Geriatrics and Gerontology }\end{array}$ & $\begin{array}{l}\text { Department of Clinical and Experimental } \\
\text { Neuroimaging }\end{array}$ \\
\hline 28 & Takashi Kato & $\begin{array}{l}\text { National Institute for Longevity Sciences, } \\
\text { National Center for Geriatrics and Gerontology }\end{array}$ & $\begin{array}{l}\text { Department of Clinical and Experimental } \\
\text { Neuroimaging }\end{array}$ \\
\hline 29 & Akinori Nakamura & $\begin{array}{l}\text { National Institute for Longevity Sciences, } \\
\text { National Center for Geriatrics and Gerontology }\end{array}$ & $\begin{array}{l}\text { Department of Clinical and Experimental } \\
\text { Neuroimaging }\end{array}$ \\
\hline 30 & Yukihiko Washimi & $\begin{array}{l}\text { National Hospital for Geriatric Medicine, } \\
\text { National Center for Geriatrics and Gerontology }\end{array}$ & Department of Neurology \\
\hline 31 & Yutaka Arahata & $\begin{array}{l}\text { National Hospital for Geriatric Medicine, } \\
\text { National Center for Geriatrics and Gerontology }\end{array}$ & Department of Neurology \\
\hline 32 & Yukihisa Miura & $\begin{array}{l}\text { National Hospital for Geriatric Medicine, } \\
\text { National Center for Geriatrics and Gerontology }\end{array}$ & Department of Outpatient Services \\
\hline 33 & Hideyuki Hattori & $\begin{array}{l}\text { National Hospital for Geriatric Medicine, } \\
\text { National Center for Geriatrics and Gerontology }\end{array}$ & Department of Psychiatry \\
\hline 34 & Yoshio Mitsuyama & Daigo Hospital & Psychogeriatric Center \\
\hline 35 & Teruhiko Inoue & Daigo Hospital & Psychogeriatric Center \\
\hline 36 & Ryuichi Ohkubo & Kagoshima University & Third Department of Internal Medicine \\
\hline 37 & Seigo Fujita & Fujimotohayasuzu Hospital & Department of Radiology \\
\hline 38 & Yasuzo Takeuchi & Fujimotohayasuzu Hospital & Department of Psychiatry \\
\hline 39 & Kenichi Meguro & $\begin{array}{l}\text { Tohoku University Graduate School of } \\
\text { Medicine }\end{array}$ & Department of Geriatric Behavioral Neurology \\
\hline 40 & Masahiro Nakatsuka & $\begin{array}{l}\text { Tohoku University Graduate School of } \\
\text { Medicine }\end{array}$ & Department of Geriatric Behavioral Neurology \\
\hline 41 & Mitsuru Ikeda & Nagoya University School of Health Sciences & Department of Radiological Technology \\
\hline
\end{tabular}


Supplementary Table 2

MRI scan acquisition parameters at the nine sites participating in the SEAD-J study.

\begin{tabular}{|c|c|c|c|c|c|c|c|c|c|}
\hline Site & Scanner & $\begin{array}{c}\text { Field } \\
\text { strength }[\mathrm{T}]\end{array}$ & Sequence & $\begin{array}{c}\mathrm{TR} \\
{[\mathrm{ms}]}\end{array}$ & $\begin{array}{c}\mathrm{TE} \\
{[\mathrm{ms}]}\end{array}$ & $\begin{array}{c}\mathrm{TI} \\
{[\mathrm{ms}]}\end{array}$ & $\begin{array}{l}\text { FOV } \\
{[\mathrm{mm}]}\end{array}$ & $\begin{array}{l}\text { Matrix } \\
\text { size }\end{array}$ & $\begin{array}{c}\text { Voxel size } \\
{\left[\mathrm{mm}^{3}\right]}\end{array}$ \\
\hline A & $\begin{array}{l}\text { MAGNETOM } \\
\text { Trio }\end{array}$ & 3.0 & MPRAGE & 2000 & 4.38 & 990 & 240 & $240 \times 256$ & $0.94 \times 0.94 \times 1.0$ \\
\hline B & Signa Excite & 1.5 & SPGR & 23 & 5 & & 220 & $256 \times 192$ & $0.78 \times 0.78 \times 1.5$ \\
\hline $\mathrm{C}$ & $\begin{array}{l}\text { MAGNETOM } \\
\text { Symphony }\end{array}$ & 1.5 & VIBE & 11 & 4.75 & & 230 & $256 \times 256$ & $1.0 \times 1.0 \times 1.0$ \\
\hline $\mathrm{D}$ & $\begin{array}{l}\text { Gyroscan } \\
\text { INTERA }\end{array}$ & 1.5 & T1-FFE & 20 & 4.6 & & 256 & $256 \times 256$ & $1.0 \times 1.0 \times 1.0$ \\
\hline $\mathrm{E}$ & $\begin{array}{l}\text { SIGNA } \\
\text { EXCITE HD }\end{array}$ & 1.5 & SPGR & 21 & 6 & & 220 & $256 \times 256$ & $1.8 \times 0.86 \times 0.86$ \\
\hline $\mathrm{F}$ & $\begin{array}{l}\text { SIGNA Echo } \\
\text { Speed }\end{array}$ & 1.5 & GR & 7.2 & 1.68 & & 220 & $256 \times 224$ & $1.0 \times 0.86 \times 0.86$ \\
\hline G & $\begin{array}{l}\text { MAGNETOM } \\
\text { Avanto }\end{array}$ & 1.5 & GR & 20 & 7 & & 250 & $256 \times 256$ & $1.4 \times 0.51 \times 0.51$ \\
\hline $\mathrm{H}$ & $\begin{array}{l}\text { MAGNETOM } \\
\text { Symphony }\end{array}$ & 1.5 & VIBE & 11 & 4.75 & & 230 & $256 \times 256$ & $1.5 \times 0.49 \times 0.49$ \\
\hline I & $\begin{array}{l}\text { MAGNETOM } \\
\text { Symphony }\end{array}$ & 1.5 & MPRAGE & 1700 & 4.18 & 800 & 230 & $256 \times 256$ & $1.5 \times 0.90 \times 0.90$ \\
\hline
\end{tabular}

TR, Repetition time; TE, Echo time; TI, Inversion time; FOV, Field of view; MPRAGE, Magnetization Prepared Rapid Gradient Echo; SPGR, Spoiled Gradient Recalled; VIBE, Volumetric Interpolated Breath hold

Examination; T1-FFE, T1-weighted Fast Field Echo; GR, Gradient Rephasing. 


\section{Supplementary Table 3}

Three-way $($ RFE $\times$ atlas $\times$ modality) ANOVA summary table.

\begin{tabular}{|c|c|c|c|c|c|c|c|}
\hline Source & $d f$ & Type II SS & $M S$ & $F$ & $p$ & & $\eta^{2}$ \\
\hline RFE & 1 & 0.0039 & 0.0039 & 20.12 & $<10^{-5}$ & $* * *$ & 0.0065 \\
\hline Atlas & 3 & 0.19 & 0.064 & 333.0 & $<10^{-15}$ & $* * *$ & 0.32 \\
\hline Modality & 2 & 0.18 & 0.089 & 466.3 & $<10^{-15}$ & $* * *$ & 0.30 \\
\hline RFE $\times$ Atlas & 3 & 0.00078 & 0.0003 & 1.347 & 0.26 & & 0.0013 \\
\hline RFE $\times$ modality & 2 & 0.00043 & 0.0002 & 1.118 & 0.33 & & 0.00072 \\
\hline Atlas $\times$ modality & 6 & 0.13 & 0.021 & 111.7 & $<10^{-15}$ & $* * *$ & 0.22 \\
\hline RFE $\times$ atlas $\times$ modality & 6 & 0.0017 & 0.0003 & 1.467 & 0.19 & & 0.0028 \\
\hline \multicolumn{8}{|l|}{ Atlas at modality } \\
\hline Atlas at MRI & 3 & 0.13 & 0.044 & 227.7 & $<10^{-15}$ & $* * *$ & 0.22 \\
\hline Atlas at PET & 3 & 0.079 & 0.026 & 137.4 & $<10^{-15}$ & $* * *$ & 0.13 \\
\hline Atlas at MRI+PET & 3 & 0.11 & 0.037 & 191.2 & $<10^{-15}$ & $* * *$ & 0.19 \\
\hline \multicolumn{8}{|l|}{ Modality at atlas } \\
\hline $\begin{array}{l}\text { Modality at } \\
\text { MRI-AAL + PET-AAL }\end{array}$ & 2 & 0.17 & 0.086 & 449.3 & $<10^{-15}$ & $* * *$ & 0.29 \\
\hline $\begin{array}{l}\text { Modality at } \\
\text { MRI-AAL + PET-LPBA40 }\end{array}$ & 2 & 0.017 & 0.0086 & 44.92 & $<10^{-15}$ & $* * *$ & 0.029 \\
\hline $\begin{array}{l}\text { Modality at } \\
\text { MRI-LPBA40 + PET-AAL }\end{array}$ & 2 & 0.039 & 0.019 & 100.5 & $<10^{-15}$ & $* * *$ & 0.065 \\
\hline $\begin{array}{l}\text { Modality at } \\
\text { MRI-LPBA40 + PET-LPBA40 }\end{array}$ & 2 & 0.079 & 0.040 & 206.6 & $<10^{-15}$ & $* * *$ & 0.13 \\
\hline Residual & 456 & 0.088 & 0.0002 & & & & \\
\hline Total & 479 & 0.59 & & & & & \\
\hline
\end{tabular}

RFE, recursive feature elimination; ANOVA, analysis of variance; df, degrees of freedom; SS, sum of squares;

MS, mean square; MRI, magnetic resonance imaging; PET, positron emission tomography; AAL, Automated Anatomical Labeling; LPBA40, LONI Probabilistic Brain Atlas.

$* p<0.05, * * p<0.01, * * * p<0.001$. 


\section{Supplementary Table 4}

Two-way (atlas $\times$ modality) ANOVA summary table.

\begin{tabular}{|c|c|c|c|c|c|c|c|}
\hline Source & $d f$ & Type II SS & $M S$ & $F$ & $p$ & & $\eta^{2}$ \\
\hline Atlas & 1 & 0.000035 & 0.000035 & 0.430 & 0.51 & & 0.0006 \\
\hline Modality & 1 & 0.00043 & 0.00043 & 5.25 & 0.025 & $*$ & 0.0078 \\
\hline Atlas $\times$ modality & 1 & 0.049 & 0.049 & 593 & $<10^{-15}$ & $* * *$ & 0.88 \\
\hline \multicolumn{8}{|l|}{ Atlas at modality } \\
\hline Atlas at MRI & 1 & 0.026 & 0.026 & 313 & $<10^{-15}$ & $* * *$ & 0.46 \\
\hline Atlas at PET & 1 & 0.023 & 0.023 & 281 & $<10^{-15}$ & $* * *$ & 0.42 \\
\hline \multicolumn{8}{|l|}{ Modality at atlas } \\
\hline Modality at AAL & 1 & 0.029 & 0.029 & 355 & $<10^{-15}$ & $* * *$ & 0.53 \\
\hline Modality at LPBA40 & 1 & 0.020 & 0.020 & 244 & $<10^{-15}$ & $* * *$ & 0.36 \\
\hline Residual & 76 & 0.0062 & 0.0001 & & & & \\
\hline Total & 79 & 0.055 & & & & & \\
\hline
\end{tabular}

ANOVA, analysis of variance; df, degrees of freedom; SS, sum of squares; MS, mean square; MRI, magnetic resonance imaging; PET, positron emission tomography; AAL, Automated Anatomical Labeling; LPBA40, LONI Probabilistic Brain Atlas.

$* p<0.05,{ }^{* *} p<0.01,{ }^{* * *} p<0.001$. 


\section{Supplementary Table 5}

Two-way (MRI atlas $\times$ PET atlas) ANOVA summary table.

\begin{tabular}{|c|c|c|c|c|c|c|c|}
\hline Source & $d f$ & Type II SS & $M S$ & $F$ & $p$ & & $\eta^{2}$ \\
\hline MRI atlas & 1 & 0.011 & 0.011 & 191.6 & $<10^{-15}$ & $* * *$ & 0.17 \\
\hline PET atlas & 1 & 0.040 & 0.040 & 716.0 & $<10^{-15}$ & $* * *$ & 0.62 \\
\hline MRI atlas $\times$ PET atlas & 1 & 0.0091 & 0.0091 & 162.3 & $<10^{-15}$ & $* * *$ & 0.14 \\
\hline \multicolumn{8}{|l|}{ MRI atlas at PET atlas } \\
\hline MRI atlas at AAL & 1 & 0.000034 & 0.000034 & 0.6048 & 0.44 & & 0.005 \\
\hline MRI atlas at LPBA40 & 1 & 0.020 & 0.020 & 353.3 & $<10^{-15}$ & $* * *$ & 0.31 \\
\hline \multicolumn{8}{|l|}{ Modality at atlas } \\
\hline PET atlas at AAL & 1 & 0.044 & 0.044 & 780.1 & $<10^{-15}$ & $* * *$ & 0.68 \\
\hline PET atlas at LPBA40 & 1 & 0.0055 & 0.0055 & 98.24 & $<10^{-15}$ & $* * *$ & 0.086 \\
\hline Residual & 76 & 0.0043 & 0.0001 & & & & \\
\hline Total & 79 & 0.064 & & & & & \\
\hline
\end{tabular}

ANOVA, analysis of variance; df, degrees of freedom; SS, sum of squares; MS, mean square; MRI, magnetic resonance imaging; PET, positron emission tomography; AAL, Automated Anatomical Labeling; LPBA40, LONI Probabilistic Brain Atlas.

$* p<0.05,{ }^{* *} p<0.01, * * * p<0.001$. 
Supplementary Table 6 ADNI participant ID (PTID) list for 158 MCI subjects.

\begin{tabular}{|c|c|c|c|c|c|}
\hline No. & PTID & Conversion & No. & PTID & Conversion \\
\hline 1 & 003_S_1057 & Converter & 78 & 003_S_1074 & Nonconverter \\
\hline 2 & 005_S_0222 & Converter & 79 & 003_S_1122 & Nonconverter \\
\hline 3 & 005_S_1224 & Converter & 80 & 005_S_0546 & Nonconverter \\
\hline 4 & 006_S_0675 & Converter & 81 & 007_S_0698 & Nonconverter \\
\hline 5 & 006_S_1130 & Converter & 82 & 010_S_0422 & Nonconverter \\
\hline 6 & 007_S_0101 & Converter & 83 & 012_S_0634 & Nonconverter \\
\hline 7 & 007_S_0128 & Converter & 84 & 013_S_1186 & Nonconverter \\
\hline 8 & 007_S_0293 & Converter & 85 & 016_S_1092 & Nonconverter \\
\hline 9 & 007_S_0344 & Converter & 86 & 018_S_0080 & Nonconverter \\
\hline 10 & 009_S_1030 & Converter & 87 & 018_S_0142 & Nonconverter \\
\hline 11 & 010_S_0161 & Converter & 88 & 021_S_0178 & Nonconverter \\
\hline 12 & 010_S_0904 & Converter & 89 & 021_S_0424 & Nonconverter \\
\hline 13 & 011_S_0326 & Converter & 90 & 021_S_0626 & Nonconverter \\
\hline 14 & 011_S_0362 & Converter & 91 & 022_S_0961 & Nonconverter \\
\hline 15 & 011_S_0861 & Converter & 92 & 027_S_0408 & Nonconverter \\
\hline 16 & 011_S_1282 & Converter & 93 & 029_S_0914 & Nonconverter \\
\hline 17 & 012_S_1033 & Converter & 94 & 029_S_1218 & Nonconverter \\
\hline 18 & 013_S_0240 & Converter & 95 & 029_S_1318 & Nonconverter \\
\hline 19 & 013_S_0325 & Converter & 96 & 029_S_1384 & Nonconverter \\
\hline 20 & 013_S_0860 & Converter & 97 & 032_S_0718 & Nonconverter \\
\hline 21 & 018_S_0057 & Converter & 98 & 035_S_0033 & Nonconverter \\
\hline 22 & 018_S_0155 & Converter & 99 & 035_S_0292 & Nonconverter \\
\hline 23 & 021_S_0141 & Converter & 100 & 036_S_0656 & Nonconverter \\
\hline 24 & 021_S_0231 & Converter & 101 & 036_S_0673 & Nonconverter \\
\hline 25 & 022_S_1351 & Converter & 102 & 036_S_0945 & Nonconverter \\
\hline 26 & 022_S_1394 & Converter & 103 & 037_S_0150 & Nonconverter \\
\hline
\end{tabular}


024_S_1393

Converter

104

105

28

29

30

31

32

33

34

35

36

37

38

39

40

41

42

43

44

45

46

47

48

49

50

51

52

53

54
027_S_0256

Converter

027_S_0461

Converter

029_S_1073

Converter

Converter

Converter

Converter

Converter

Converter

Converter

Converter

Converter

Converter

Converter

Converter

Converter

Converter

Converter

Converter

Converter

Converter

Converter

Converter

Converter

Converter

Converter

Converter

Converter
106

107

108

109

110

111

112

113

114

115

116

117

118

119

120

121

122

123

124

125

126

127

128

129

130

131
037_S_0377 Nonconverter

037_S_1078 Nonconverter

037_S_1421 Nonconverter

041_S_0679 Nonconverter

041_S_1260 Nonconverter

041_S_1418 Nonconverter

052_S_1346 Nonconverter

053_S_0389 Nonconverter

053_S_0621 Nonconverter

053_S_0919 Nonconverter

057_S_0464 Nonconverter

072_S_1211 Nonconverter

072_S_1380 Nonconverter

073_S_0746 Nonconverter

094_S_1188 Nonconverter

094_S_1314 Nonconverter

094_S_1417 Nonconverter

098_S_0160 Nonconverter

099_S_0051 Nonconverter

099_S_0291 Nonconverter

099_S_0551 Nonconverter

099_S_1034 Nonconverter

109_S_0950 Nonconverter

109_S_1114 Nonconverter

114_S_0378 Nonconverter

114_S_1106 Nonconverter

114_S_1118 Nonconverter

116_S_0361 Nonconverter 
55

56

57

58

59

60

61

62

63

64

65

66

67

68

69

70

71

72

73

74

75

76

77
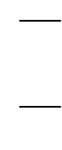

073_S_0909 Converter

Converter

Converter

Converter

Converter

Converter

Converter

Converter

Converter

Converter

Converter

Converter

Converter

Converter

Converter

Converter

Converter

Converter

Converter

Converter

Converter

Converter

Converter

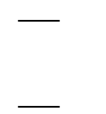

132

133

134

135

136

137

138

139

140

141

142

143

144

145

146

147

148

149

150

151

152

153

154

155

156

157

158
126_S_0709 Nonconverter

127_S_0112 Nonconverter

127_S_0925 Nonconverter

127_S_1032 Nonconverter

127_S_1419 Nonconverter

128_S_0135 Nonconverter

128_S_0138 Nonconverter

128_S_0188 Nonconverter

128_S_0200 Nonconverter

128_S_0205 Nonconverter

128_S_0225 Nonconverter

128_S_0608 Nonconverter

128_S_0715 Nonconverter

128_S_0770 Nonconverter

128_S_1406 Nonconverter

128_S_1408 Nonconverter

129_S_1246 Nonconverter

130_S_0285 Nonconverter

137_S_0158 Nonconverter

137_S_0443 Nonconverter

137_S_0481 Nonconverter

137_S_0669 Nonconverter

137_S_0722 Nonconverter

137_S_0800 Nonconverter

137_S_0994 Nonconverter

137_S_1414 Nonconverter

141_S_1378 Nonconverter 
Supplementary Table 7 Baseline characteristics of ADNI subjects.

\begin{tabular}{lcccc}
\hline Measure & MCI-C $(\mathrm{n}=77)$ & MCI-NC $(\mathrm{n}=81)$ & $p$-value & Effect size \\
\hline Male (n, \%) & $46(59.7)$ & $58(71.6)$ & $0.16^{\dagger}$ & $0.11^{\ddagger}$ \\
Age (years) & $74.8 \pm 7.0$ & $75.0 \pm 7.7$ & 0.85 & 0.02 \\
Education (years) & $16.0 \pm 2.8$ & $15.7 \pm 2.6$ & 0.57 & 0.05 \\
MMSE & $26.8 \pm 1.7$ & $27.5 \pm 1.6$ & $0.0096^{* *}$ & 0.21 \\
ADAS-cog & $12.8 \pm 3.9$ & $8.8 \pm 3.7$ & $<0.001^{* * *}$ & 0.47 \\
\hline
\end{tabular}

Converters developed Alzheimer's disease within 36 months after inclusion. MCI, mild cognitive impairment; MCI-C, MCI converter; MCI-NC, MCI nonconverter; ADNI, Alzheimer's Disease Neuroimaging Initiative; MMSE, MMSE, Mini-Mental State Examination; ADAS-cog, Alzheimer’s Disease Assessment Scale-Cognitive Subscale. Student's $t$-test unless otherwise indicated, ${ }^{* *} p<0.01,{ }^{* * *} p<0.001$.

${ }^{\dagger} \chi^{2}$ test.

$\ddagger \mathrm{w}$. 
Supplementary Table 8 Three-way (RFE $\times$ atlas $\times$ modality) ANOVA summary table for the ADNI dataset.

\begin{tabular}{|c|c|c|c|c|c|c|c|c|}
\hline Source & $d f$ & Type & II $S S$ & MS & $F$ & $p$ & & $\eta^{2}$ \\
\hline RFE & 1 & & 0.4028 & 0.4028 & $2529.7<$ & 0.001 & $* * *$ & 0.488 \\
\hline Atlas & 3 & & 0.0604 & 0.0201 & $126.5<$ & 0.001 & $* * *$ & 0.073 \\
\hline Modality & 2 & & 0.0787 & 0.0394 & $247.2<$ & 0.001 & $* * *$ & 0.095 \\
\hline RFE $\times$ Atlas & 3 & & 0.0670 & 0.0223 & $140.3<$ & 0.001 & $* * *$ & 0.081 \\
\hline RFE $\times$ modality & 2 & & 0.0632 & 0.0316 & $198.6<$ & 0.001 & $* * *$ & 0.077 \\
\hline Atlas $\times$ modality & 6 & & 0.0396 & 0.0066 & $41.4<$ & 0.001 & $* * *$ & 0.048 \\
\hline RFE $\times$ atlas $\times$ modality & 6 & & 0.0406 & 0.0068 & $42.5<$ & 0.001 & $* * *$ & 0.049 \\
\hline Residual & 456 & & 0.0726 & 0.0002 & & & & \\
\hline Total & 479 & & 0.8251 & & & & & \\
\hline
\end{tabular}

RFE, recursive feature elimination; ANOVA, analysis of variance; ADNI, Alzheimer's

Disease Neuroimaging Initiative; df, degrees of freedom; SS, sum of squares; MS, mean square.

${ }^{*} p<0.05,{ }^{* *} p<0.01, * * * p<0.001$. 
Supplementary Table 9 ADNI Two-way ANOVA summary table: RFE $\times$ atlas interaction at each level of factor modality.

\begin{tabular}{lrrrrrrr}
\hline \multicolumn{1}{c}{ Source } & $d f$ & Type II SS & MS & \multicolumn{1}{c}{$p$} & & $\eta^{2}$ \\
\hline RFE $\times$ atlas at MRI & & & & & & & \\
RFE & 1 & 0.1635 & 0.1635 & $1515<0.001$ & $* * *$ & 0.84 \\
Atlas & 1 & 0.0034 & 0.0034 & $31.6<0.001$ & $* * *$ & 0.018 \\
RFE $\times$ atlas & 1 & 0.0195 & 0.0195 & $180.8<0.001$ & $* * *$ & 0.10 \\
Residual & 76 & 0.0082 & 0.0001 & & & \\
Total & 79 & 0.1946 & & & & \\
RFE $\times$ atlas at PET & & & & & & \\
RFE & 1 & 0.0352 & 0.0352 & $338.9<0.001$ & $* * *$ & 0.61 \\
Atlas & 1 & 0.0108 & 0.0108 & $104.4<0.001$ & $* * *$ & 0.19 \\
RFE $\times$ atlas & 1 & 0.0040 & 0.0040 & $38.8<0.001$ & $* * *$ & 0.070 \\
Residual & 76 & 0.0079 & 0.0001 & & & \\
Total & 79 & 0.0579 & & & & \\
RFE $\times$ atlas at MRI+PET & & & & & & \\
RFE & 1 & 0.0688 & 0.0688 & $258.7<0.001$ & $* * *$ & 0.29 \\
Atlas & 3 & 0.0715 & 0.0238 & $89.6<0.001$ & $* * *$ & 0.30 \\
RFE $\times$ atlas & 3 & 0.0606 & 0.0202 & $75.9<0.001$ & $* * *$ & 0.25 \\
Residual & 152 & 0.0404 & 0.0003 & & & \\
Total & 159 & 0.2413 & & & & \\
\hline ADNI Alzheimer & & & & & & \\
\end{tabular}

ADNI, Alzheimer's Disease Neuroimaging Initiative; ANOVA, analysis of variance; RFE, recursive feature elimination; df, degrees of freedom; SS, sum of squares; MS, mean square; MRI, magnetic resonance imaging; PET, positron emission tomography.

$* p<0.05, * * p<0.01, * * * p<0.001$. 
Supplementary Table 10 ADNI Two-way ANOVA summary table: RFE × modality interaction at each level of factor atlas.

\begin{tabular}{|c|c|c|c|c|c|c|}
\hline Source & $d f$ & Type II SS & $M S$ & $F$ & & $\eta^{2}$ \\
\hline \multicolumn{7}{|c|}{$\mathrm{RFE} \times$ modality at $\mathrm{AAL}$} \\
\hline RFE & 1 & 0.0572 & 0.0572 & $242.0<0.001$ & $* * *$ & 0.43 \\
\hline Modality & 2 & 0.0371 & 0.0185 & $78.5<0.001$ & $* * *$ & 0.28 \\
\hline RFE $\times$ modality & 2 & 0.0118 & 0.0059 & $25.0<0.001$ & $* * *$ & 0.089 \\
\hline Residual & 114 & 0.0269 & 0.0002 & & & \\
\hline Total & 119 & 0.1330 & & & & \\
\hline \multicolumn{7}{|l|}{$\begin{array}{l}\text { RFE } \times \text { modality at } \\
\text { AAL }+ \text { LPBA } 40\end{array}$} \\
\hline RFE & 1 & 0.0318 & 0.0318 & $348.2<0.001$ & $* * *$ & 0.50 \\
\hline Modality & 2 & 0.0087 & 0.0044 & $47.9<0.001$ & $* * *$ & 0.14 \\
\hline RFE $\times$ modality & 2 & 0.0121 & 0.0060 & $66.1<0.001$ & $* * *$ & 0.19 \\
\hline Residual & 114 & 0.0104 & 0.0001 & & & \\
\hline Total & 119 & 0.0630 & & & & \\
\hline \multicolumn{7}{|l|}{$\begin{array}{l}\text { RFE } \times \text { modality at } \\
\text { LPBA } 40+A A L\end{array}$} \\
\hline RFE & 1 & 0.2713 & 0.2713 & $1395.1<0.001$ & $* * *$ & 0.78 \\
\hline Modality & 2 & 0.0283 & 0.0141 & $72.6<0.001$ & $* * *$ & 0.082 \\
\hline RFE $\times$ modality & 2 & 0.0238 & 0.0119 & $61.1<0.001$ & $* * *$ & 0.069 \\
\hline Residual & 114 & 0.0222 & 0.0002 & & & \\
\hline Total & 119 & 0.3455 & & & & \\
\hline \multicolumn{7}{|c|}{ RFE $\times$ modality at LPBA40 } \\
\hline RFE & 1 & 0.1097 & 0.1097 & $953.4<0.001$ & $* * *$ & 0.49 \\
\hline Modality & 2 & 0.0442 & 0.0221 & $192.2<0.001$ & $* * *$ & 0.20 \\
\hline RFE $\times$ modality & 2 & 0.0562 & 0.0281 & $244.5<0.001$ & $* * *$ & 0.25 \\
\hline Residual & 114 & 0.0131 & 0.0001 & & & \\
\hline Total & 119 & 0.2232 & & & & \\
\hline
\end{tabular}

ADNI, Alzheimer's Disease Neuroimaging Initiative; ANOVA, analysis of variance; df, degrees of freedom; RFE, recursive feature elimination; SS, sum of squares; MS, mean square; AAL, Automated Anatomical Labeling; LPBA40, LONI Probabilistic Brain Atlas. $* p<0.05, * * p<0.01, * * * p<0.001$. 
Supplementary Table 11 ADNI Two-way ANOVA summary table: atlas × modality interaction at each level of factor RFE.

\begin{tabular}{lrrrrrrr}
\hline \multicolumn{1}{c}{ Source } & $d f$ & Type II SS & MS & \multicolumn{1}{c}{$F$} & $p$ & & $\eta^{2}$ \\
\hline Atlas $\times$ modality at RFE- & & & & & & & \\
Atlas & 3 & 0.1106 & 0.0369 & $688.1<0.001$ & $* * *$ & 0.44 \\
Modality & 2 & 0.0702 & 0.0351 & $655.2<0.001$ & $* * *$ & 0.28 \\
Atlas $\times$ modality & 6 & 0.0578 & 0.0096 & $179.9<0.001$ & $* * *$ & 0.23 \\
Residual & 228 & 0.0122 & 0.0001 & & & \\
Total & 239 & 0.2507 & & & & \\
Atlas $\times$ modality at RFE+ & & & & & & \\
Atlas & 3 & 0.0169 & 0.0056 & $21.3<0.001$ & $* * *$ & 0.10 \\
Modality & 2 & 0.0718 & 0.0359 & $135.5<0.001$ & $* * *$ & 0.42 \\
Atlas $\times$ modality & 6 & 0.0224 & 0.0037 & $14.1<0.001$ & $* * *$ & 0.13 \\
Residual & 228 & 0.0604 & 0.0003 & & & \\
Total & 239 & 0.1715 & & & & \\
\hline ADNI, Alzhimer
\end{tabular}

degrees of freedom; RFE, recursive feature elimination; SS, sum of squares; MS, mean square.

$* p<0.05, * * p<0.01, * * * p<0.001$. 
Supplementary Table 12 ADNI Two-way ANOVA summary table: simple main effects for

RFE $\times$ atlas interaction at each level of factor modality.

\begin{tabular}{|c|c|c|c|c|c|c|c|}
\hline Source & $d f$ & Type II SS & $M S$ & $F$ & $p$ & & $\eta^{2}$ \\
\hline \multicolumn{8}{|l|}{$\mathrm{RFE} \times$ atlas at MRI } \\
\hline RFE at AAL & 1 & 0.0350 & 0.0350 & $324.4<$ & 0.001 & $* * *$ & 0.18 \\
\hline RFE at LPBA40 & 1 & 0.1480 & 0.1480 & $1370.9<$ & 0.001 & $* * *$ & 0.76 \\
\hline Atlas at RFE- & 1 & 0.0196 & 0.0196 & $181.8<$ & 0.001 & $* * *$ & 0.10 \\
\hline Atlas at RFE+ & 1 & 0.0033 & 0.0033 & $30.6<$ & 0.001 & $* * *$ & 0.017 \\
\hline Residual & 76 & 0.0082 & 0.0001 & & & & \\
\hline Total & 79 & 0.1946 & & & & & \\
\hline \multicolumn{8}{|l|}{$\mathrm{RFE} \times$ atlas at PET } \\
\hline RFE at AAL & 1 & 0.0315 & 0.0315 & $303.5<$ & 0.001 & $* * *$ & 0.54 \\
\hline RFE at LPBA40 & 1 & 0.0077 & 0.0077 & $74.2<$ & 0.001 & $* * *$ & 0.13 \\
\hline Atlas at RFE- & 1 & 0.0140 & 0.0140 & $135.3<$ & 0.001 & $* * *$ & 0.24 \\
\hline Atlas at RFE+ & 1 & 0.0008 & 0.0008 & 8.0 & 0.0061 & $* *$ & 0.014 \\
\hline Residual & 76 & 0.0079 & 0.0001 & & & & \\
\hline Total & 79 & 0.0579 & & & & & \\
\hline \multicolumn{8}{|l|}{$\mathrm{RFE} \times$ atlas at $\mathrm{MRI}+\mathrm{PET}$} \\
\hline RFE at AAL & 1 & 0.0024 & 0.0024 & 9.2 & 0.0028 & $* *$ & 0.010 \\
\hline RFE at AAL+LPBA40 & 1 & 0.0012 & 0.0012 & 4.4 & 0.039 & $*$ & 0.0048 \\
\hline RFE at LPBA40+AAL & 1 & 0.1156 & 0.1156 & $434.5<$ & 0.001 & $* * *$ & 0.48 \\
\hline RFE at LPBA40 & 1 & 0.0102 & 0.0102 & $38.5<$ & 0.001 & $* * *$ & 0.042 \\
\hline Atlas at RFE- & 3 & 0.1010 & 0.0337 & $126.6<$ & 0.001 & $* * *$ & 0.42 \\
\hline Atlas at RFE+ & 3 & 0.0311 & 0.0104 & $38.9<$ & 0.001 & $* * *$ & 0.13 \\
\hline Residual & 152 & 0.0404 & 0.0003 & & & & \\
\hline Total & 159 & 0.2413 & & & & & \\
\hline
\end{tabular}

ADNI, Alzheimer's Disease Neuroimaging Initiative; ANOVA, analysis of variance; RFE, recursive feature elimination; df, degrees of freedom; SS, sum of squares; MS, mean square; MRI, magnetic resonance imaging; PET, positron emission tomography.

$* p<0.05, * * p<0.01, * * * p<0.001$. 
Supplementary Table 13 ADNI Two-way ANOVA summary table: simple main effects for

RFE $\times$ modality interaction at each level of factor atlas.

\begin{tabular}{|c|c|c|c|c|c|c|}
\hline Source & $d f$ & Type II SS & MS & $F$ & & $\eta^{2}$ \\
\hline \multicolumn{7}{|c|}{$\mathrm{RFE} \times$ modality at AAL } \\
\hline RFE at MRI & 1 & 0.0350 & 0.0350 & $148.2<0.001$ & $* * *$ & 0.26 \\
\hline RFE at PET & 1 & 0.0315 & 0.0315 & $133.4<0.001$ & $* * *$ & 0.24 \\
\hline RFE at MRI+PET & 1 & 0.0024 & 0.0024 & $10.4 \quad 0.0017$ & $* *$ & 0.018 \\
\hline Modality at RFE- & 2 & 0.0035 & 0.0018 & $7.4<0.001$ & $* * *$ & 0.027 \\
\hline Modality at RFE+ & 2 & 0.0454 & 0.0227 & $96.0<0.001$ & $* * *$ & 0.34 \\
\hline Residual & 114 & 0.0269 & 0.0002 & & & \\
\hline Total & 119 & 0.1330 & & & & \\
\hline \multicolumn{7}{|l|}{$\begin{array}{l}\text { RFE } \times \text { modality at } \\
\text { AAL }+ \text { LPBA40 }\end{array}$} \\
\hline RFE at MRI & 1 & 0.0350 & 0.0350 & $383.4<0.001$ & $* * *$ & 0.56 \\
\hline RFE at PET & 1 & 0.0077 & 0.0077 & $84.3<0.001$ & $* * *$ & 0.12 \\
\hline $\mathrm{RFE}$ at MRI+PET & 1 & 0.0012 & 0.0012 & $12.7<0.001$ & $* * *$ & 0.018 \\
\hline Modality at RFE- & 2 & 0.0180 & 0.0090 & $98.6<0.001$ & $* * *$ & 0.29 \\
\hline Modality at RFE+ & 2 & 0.0028 & 0.0014 & $15.3<0.001$ & $* * *$ & 0.044 \\
\hline Residual & 114 & 0.0104 & 0.0001 & & & \\
\hline Total & 119 & 0.0630 & & & & \\
\hline \multicolumn{7}{|l|}{$\begin{array}{l}\text { RFE } \times \text { modality at } \\
\text { LPBA40+AAL }\end{array}$} \\
\hline RFE at MRI & 1 & 0.1480 & 0.1480 & $760.9<0.001$ & $* * *$ & 0.43 \\
\hline RFE at PET & 1 & 0.0315 & 0.0315 & $162.0<0.001$ & $* * *$ & 0.091 \\
\hline RFE at MRI+PET & 1 & 0.1156 & 0.1156 & $594.3<0.001$ & $* * *$ & 0.33 \\
\hline Modality at RFE- & 2 & 0.0406 & 0.0203 & $104.5<0.001$ & $* * *$ & 0.12 \\
\hline Modality at RFE+ & 2 & 0.0114 & 0.0057 & $29.3<0.001$ & $* * *$ & 0.033 \\
\hline Residual & 114 & 0.0222 & 0.0002 & & & \\
\hline Total & 119 & 0.3455 & & & & \\
\hline \multicolumn{7}{|c|}{ RFE $\times$ modality at LPBA40 } \\
\hline RFE at MRI & 1 & 0.1480 & 0.1480 & $1286.4<0.001$ & $* * *$ & 0.66 \\
\hline RFE at PET & 1 & 0.0077 & 0.0077 & $66.9<0.001$ & $* * *$ & 0.035 \\
\hline RFE at MRI+PET & 1 & 0.0102 & 0.0102 & $89.0<0.001$ & $* * *$ & 0.046 \\
\hline Modality at RFE- & 2 & 0.0658 & 0.0329 & $286.1<0.001$ & $* * *$ & 0.29 \\
\hline Modality at RFE+ & 2 & 0.0346 & 0.0173 & $150.6<0.001$ & $* * *$ & 0.16 \\
\hline Residual & 114 & 0.0131 & 0.0001 & & & \\
\hline
\end{tabular}


Total

119

0.2232

ADNI, Alzheimer’s Disease Neuroimaging Initiative; ANOVA, analysis of variance; RFE, recursive feature elimination; df, degrees of freedom; SS, sum of squares; MS, mean square; MRI, magnetic resonance imaging; PET, positron emission tomography; AAL, Automated Anatomical Labeling; LPBA40, LONI Probabilistic Brain Atlas.

$* p<0.05,{ }^{* *} p<0.01, * * * p<0.001$. 
Supplementary Table 14 ADNI Two-way ANOVA summary table: simple main effects for atlas $\times$ modality interaction at each level of factor RFE.

\begin{tabular}{lrrrrrrr}
\hline \multicolumn{1}{c}{ Source } & $d f$ & Type II SS & \multicolumn{1}{c}{$M S$} & \multicolumn{1}{c}{$p$} & & $\eta^{2}$ \\
\hline Atlas $\times$ modality at RFE- & & & & & & \\
Atlas at MRI & 3 & 0.0393 & 0.0131 & $244.3<0.001$ & $* * *$ & 0.16 \\
Atlas at PET & 3 & 0.0281 & 0.0094 & $174.8<0.001$ & $* * *$ & 0.11 \\
Atlas at MRI+PET & 3 & 0.1010 & 0.0337 & $628.7<0.001$ & $* * *$ & 0.40 \\
Modality at AAL & 2 & 0.0035 & 0.0018 & $32.8<0.001$ & $* * *$ & 0.014 \\
Modality at AAL+LPBA40 & 2 & 0.0180 & 0.0090 & $168.2<0.001$ & $* * *$ & 0.072 \\
Modality at LPBA40+AAL & 2 & 0.0406 & 0.0203 & $379.4<0.001$ & $* * *$ & 0.16 \\
Modality at LPBA40 & 2 & 0.0658 & 0.0329 & $614.4<0.001$ & $* * *$ & 0.26 \\
Residual & 228 & 0.0122 & 0.0001 & & & \\
Total & 239 & 0.2507 & & & & \\
Atlas $\times$ modality at RFE+ & & & & & & \\
Atlas at MRI & 3 & 0.0066 & 0.0022 & $8.3<0.001$ & $* * *$ & 0.039 \\
Atlas at PET & 3 & 0.0017 & 0.0006 & 2.1 & 0.10 & NS & 0.0096 \\
Atlas at MRI+PET & 3 & 0.0311 & 0.0104 & $39.1<0.001$ & $* * *$ & 0.18 \\
Modality at AAL & 2 & 0.0454 & 0.0227 & $85.6<0.001$ & $* * *$ & 0.26 \\
Modality at AAL+LPBA40 & 2 & 0.0280 & 0.0014 & 5.3 & 0.0057 & $* *$ & 0.016 \\
Modality at LPBA40+AAL & 2 & 0.0114 & 0.0057 & $21.5<0.001$ & $* * *$ & 0.066 \\
Modality at LPBA40 & 2 & 0.0346 & 0.0173 & $65.4<0.001$ & $* * *$ & 0.20 \\
Residual & 228 & 0.0604 & 0.0003 & & & \\
Total & 239 & 0.1715 & & & & \\
\hline ADNI Alzheimers Disease New0 & & & & & \\
\end{tabular}

ADNI, Alzheimer's Disease Neuroimaging Initiative; ANOVA, analysis of variance; RFE, recursive feature elimination; df, degrees of freedom; SS, sum of squares; MS, mean square; MRI, magnetic resonance imaging; PET, positron emission tomography; AAL, Automated Anatomical Labeling; LPBA40, LONI Probabilistic Brain Atlas.

$* p<0.05,{ }^{* *} p<0.01, * * * p<0.001$. 